\title{
Estimating Systematic Error and Uncertainty in Ab Initio Thermochemistry: I. Atomization Energies of Hydrocarbons in the ATOMIC(hc) Protocol
}

Dirk Bakowies

June 19, 2019

\section{Supporting Information}

Please note that all literature references and references to Figures, Tables, and Sections with numerical label (without preceding "S") apply to the bibliography and material included in the paper. Basis set names are at times abbreviated, compare footnotes to Table 3 as well as Section 3. 


\section{List of Figures}

$\mathrm{S} 1 \quad$ Set of molecules, part $1 \ldots \ldots \ldots \ldots$

S2 Set of molecules, part $2 \ldots \ldots \ldots \ldots$

S3 Set of molecules, part $3 \ldots \ldots \ldots$

S4 Set of molecules, part $4 \ldots \ldots \ldots \ldots$

S5 Set of molecules, part $5 \ldots \ldots \ldots \ldots \ldots$

S6 Set of molecules, part $6 \ldots \ldots \ldots \ldots \ldots$

S7 Set of molecules, part $7 \ldots \ldots \ldots \ldots \ldots$

S8 Set of molecules, part $8 \ldots \ldots \ldots \ldots \ldots$

S9 Set of molecules, part $9 \ldots \ldots \ldots \ldots \ldots$

S10 Set of molecules, part $10 \ldots \ldots \ldots \ldots \ldots$

\section{List of Tables}

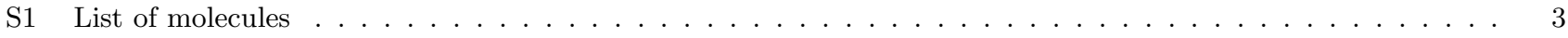

S2 CCSD valence-shell correlation contributions to the atomization energy at the complete basis set limit . . . . 16

S3 CCSD valence-shell correlation contributions to the atomization energy . . . . . . . . . . . . . . . . . 17

S4 All-electron correlation contributions to the atomization energy . . . . . . . . . . . . . . . 18

S5 Effective extrapolation exponents for CCSD/cc-pVXZ valence shell correlation contributions to atomization

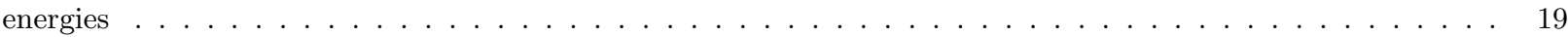

S6 Effective extrapolation exponents for CCSD/cc-pCVXZ valence shell correlation contributions to atomization

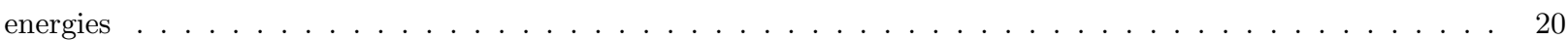

S7 Effective extrapolation exponents for CCSD(full)/cc-pCVXZ correlation contributions to atomization energies

S8 Effective extrapolation exponents for $\operatorname{CCSD}(\mathrm{T})($ full)/cc-pCVXZ correlation contributions to atomization en-

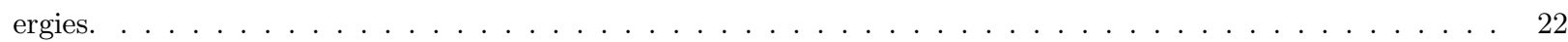

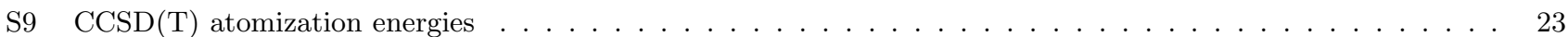

S10 Higher order electron correlation contributions to atomization energies (non-extrapolated) . . . . . . . . . . 24

S11 Higher order electron correlation contributions to atomization energies (CBS-extrapolated) . . . . . . . . 34

S12 Higher order electron correlation contributions to bond separation energies (non-extrapolated) . . . . . . . . 42

S13 Higher order electron correlation contributions to bond separation energies (CBS-extrapolated) . . . . . . . . 51

S14 Higher order electron correlation contributions to atomization energies: Summary . . . . . . . . . . . 58

S15 Electron correlation diagnostics . . . . . . . . . . . . . . . . . . . . . . . . . . . . 61

S16 Differences between ROHF-based and UHF-based total energies for the carbon atom (part 1) . . . . . . . . 64

S17 Differences between ROHF-based and UHF-based total energies for the carbon atom (part 2) . . . . . . . . 65

S18 Scalar relativistic contributions to atomization energies . . . . . . . . . . . . . . . . . . . 66

S19 DBOC contributions to atomization energies . . . . . . . . . . . . . . . . . . . 69

S20 DBOC for homologous series of hydrocarbons . . . . . . . . . . . . . . . . . . . . . . 72

$\mathrm{S} 21$ Atomization energies of small hydrocarbons . . . . . . . . . . . . . . . . . . . . . . . 73 
Table S1: List of molecules

\begin{tabular}{|c|c|c|c|c|c|c|}
\hline & \multirow[t]{2}{*}{ Molecule } & \multirow[t]{2}{*}{ Point Group } & \multicolumn{4}{|c|}{ Number of bonds } \\
\hline & & & ${ }^{3} \mathrm{C}-\mathrm{H}$ & ${ }^{3} \mathrm{C}-\mathrm{C}^{3}$ & ${ }^{2} \mathrm{C}=\mathrm{C}^{2}$ & ${ }^{1} \mathrm{C} \equiv \mathrm{C}^{1}$ \\
\hline $\mathrm{CH}_{4}$ & methane & $\mathrm{T}_{\mathrm{d}}$ & 4 & 0 & 0 & 0 \\
\hline $\mathrm{C}_{2} \mathrm{H}_{2}$ & acetylene & $\mathrm{D}_{\infty \mathrm{h}}$ & 2 & 0 & 0 & 1 \\
\hline $\mathrm{C}_{2} \mathrm{H}_{4}$ & ethylene & $\mathrm{D}_{2 \mathrm{~h}}$ & 4 & 0 & 1 & 0 \\
\hline $\mathrm{C}_{2} \mathrm{H}_{6}$ & ethane & $\mathrm{D}_{3 \mathrm{~d}}$ & 6 & 1 & 0 & 0 \\
\hline $\mathrm{C}_{3} \mathrm{H}_{4}$ & allene & $\mathrm{D}_{2 \mathrm{~d}}$ & 4 & 0 & 2 & 0 \\
\hline $\mathrm{C}_{3} \mathrm{H}_{4}$ & cyclopropene & $\mathrm{C}_{2 \mathrm{v}}$ & 4 & 2 & 1 & 0 \\
\hline $\mathrm{C}_{3} \mathrm{H}_{4}$ & propyne & $\mathrm{C}_{3 \mathrm{v}}$ & 4 & 1 & 0 & 1 \\
\hline $\mathrm{C}_{3} \mathrm{H}_{6}$ & cyclopropane & $\mathrm{D}_{3 \mathrm{~h}}$ & 6 & 3 & 0 & 0 \\
\hline $\mathrm{C}_{3} \mathrm{H}_{6}$ & propene & $\mathrm{C}_{\mathrm{s}}$ & 6 & 1 & 1 & 0 \\
\hline $\mathrm{C}_{3} \mathrm{H}_{8}$ & propane & $\mathrm{C}_{2 \mathrm{v}}$ & 8 & 2 & 0 & 0 \\
\hline $\mathrm{C}_{4} \mathrm{H}_{2}$ & 1,3-butadiyne & $\mathrm{D}_{\infty \mathrm{h}}$ & 2 & 1 & 0 & 2 \\
\hline $\mathrm{C}_{4} \mathrm{H}_{4}$ & bicyclo[1.1.0]but-1(3)-ene & $\mathrm{C}_{2 \mathrm{v}}$ & 4 & 4 & 1 & 0 \\
\hline $\mathrm{C}_{4} \mathrm{H}_{4}$ & butatriene & $\mathrm{D}_{2 \mathrm{~h}}$ & 4 & 0 & 3 & 0 \\
\hline $\mathrm{C}_{4} \mathrm{H}_{4}$ & cyclobutadiene & $\mathrm{D}_{2 \mathrm{~h}}$ & 4 & 2 & 2 & 0 \\
\hline $\mathrm{C}_{4} \mathrm{H}_{4}$ & ethynylethene & $\mathrm{C}_{\mathrm{s}}$ & 4 & 1 & 1 & 1 \\
\hline $\mathrm{C}_{4} \mathrm{H}_{4}$ & methylenecyclopropene & $\mathrm{C}_{2 \mathrm{v}}$ & 4 & 2 & 2 & 0 \\
\hline $\mathrm{C}_{4} \mathrm{H}_{4}$ & tetrahedrane & $\mathrm{T}_{\mathrm{d}}$ & 4 & 6 & 0 & 0 \\
\hline $\mathrm{C}_{4} \mathrm{H}_{6}$ & 1,2-butadiene & $\mathrm{C}_{\mathrm{s}}$ & 6 & 1 & 2 & 0 \\
\hline $\mathrm{C}_{4} \mathrm{H}_{6}$ & 1,3-butadiene & $\mathrm{C}_{2 \mathrm{~h}}$ & 6 & 1 & 2 & 0 \\
\hline $\mathrm{C}_{4} \mathrm{H}_{6}$ & 2-butyne & $\mathrm{D}_{3 \mathrm{~h}}$ & 6 & 2 & 0 & 1 \\
\hline $\mathrm{C}_{4} \mathrm{H}_{6}$ & bicyclo[1.1.0]butane & $\mathrm{C}_{2 \mathrm{v}}$ & 6 & 5 & 0 & 0 \\
\hline $\mathrm{C}_{4} \mathrm{H}_{6}$ & cyclobutene & $\mathrm{C}_{2 \mathrm{v}}$ & 6 & 3 & 1 & 0 \\
\hline $\mathrm{C}_{4} \mathrm{H}_{6}$ & methylenecyclopropane & $\mathrm{C}_{2 \mathrm{v}}$ & 6 & 3 & 1 & 0 \\
\hline $\mathrm{C}_{4} \mathrm{H}_{8}$ & cyclobutane & $\mathrm{C}_{2 \mathrm{v}}$ & 8 & 4 & 0 & 0 \\
\hline $\mathrm{C}_{4} \mathrm{H}_{8}$ & isobutene & $\mathrm{C}_{2 \mathrm{v}}$ & 8 & 2 & 1 & 0 \\
\hline $\mathrm{C}_{4} \mathrm{H}_{10}$ & isobutane & $\mathrm{C}_{3 \mathrm{v}}$ & 10 & 3 & 0 & 0 \\
\hline $\mathrm{C}_{4} \mathrm{H}_{10}$ & $n$-butane & $\mathrm{C}_{2 \mathrm{~h}}$ & 10 & 3 & 0 & 0 \\
\hline $\mathrm{C}_{5} \mathrm{H}_{4}$ & 1,4-pentadiyne & $\mathrm{C}_{2 \mathrm{v}}$ & 4 & 2 & 0 & 2 \\
\hline $\mathrm{C}_{5} \mathrm{H}_{4}$ & pentatetraene & $\mathrm{D}_{2 \mathrm{~d}}$ & 4 & 0 & 4 & 0 \\
\hline $\mathrm{C}_{5} \mathrm{H}_{4}$ & spiropentadiene & $\mathrm{D}_{2 \mathrm{~d}}$ & 4 & 4 & 2 & 0 \\
\hline $\mathrm{C}_{5} \mathrm{H}_{6}$ & [1.1.1]propellane & $\mathrm{D}_{3 \mathrm{~h}}$ & 6 & 7 & 0 & 0 \\
\hline $\mathrm{C}_{5} \mathrm{H}_{6}$ & 1,3-cyclopentadiene & $\mathrm{C}_{2 \mathrm{v}}$ & 6 & 3 & 2 & 0 \\
\hline $\mathrm{C}_{5} \mathrm{H}_{6}$ & 3-methylenecyclobutene & $\mathrm{C}_{\mathrm{s}}$ & 6 & 3 & 2 & 0 \\
\hline $\mathrm{C}_{5} \mathrm{H}_{6}$ & bicyclo[2.1.0]pent-2-ene & $\mathrm{C}_{\mathrm{s}}$ & 6 & 5 & 1 & 0 \\
\hline $\mathrm{C}_{5} \mathrm{H}_{6}$ & cyclopropylacetylene & $\mathrm{C}_{\mathrm{s}}$ & 6 & 4 & 0 & 1 \\
\hline $\mathrm{C}_{5} \mathrm{H}_{8}$ & 1,4-pentadiene & $\mathrm{C}_{2}$ & 8 & 2 & 2 & 0 \\
\hline $\mathrm{C}_{5} \mathrm{H}_{8}$ & isoprene & $\mathrm{C}_{\mathrm{s}}$ & 8 & 2 & 2 & 0 \\
\hline
\end{tabular}


Table S1, contd.

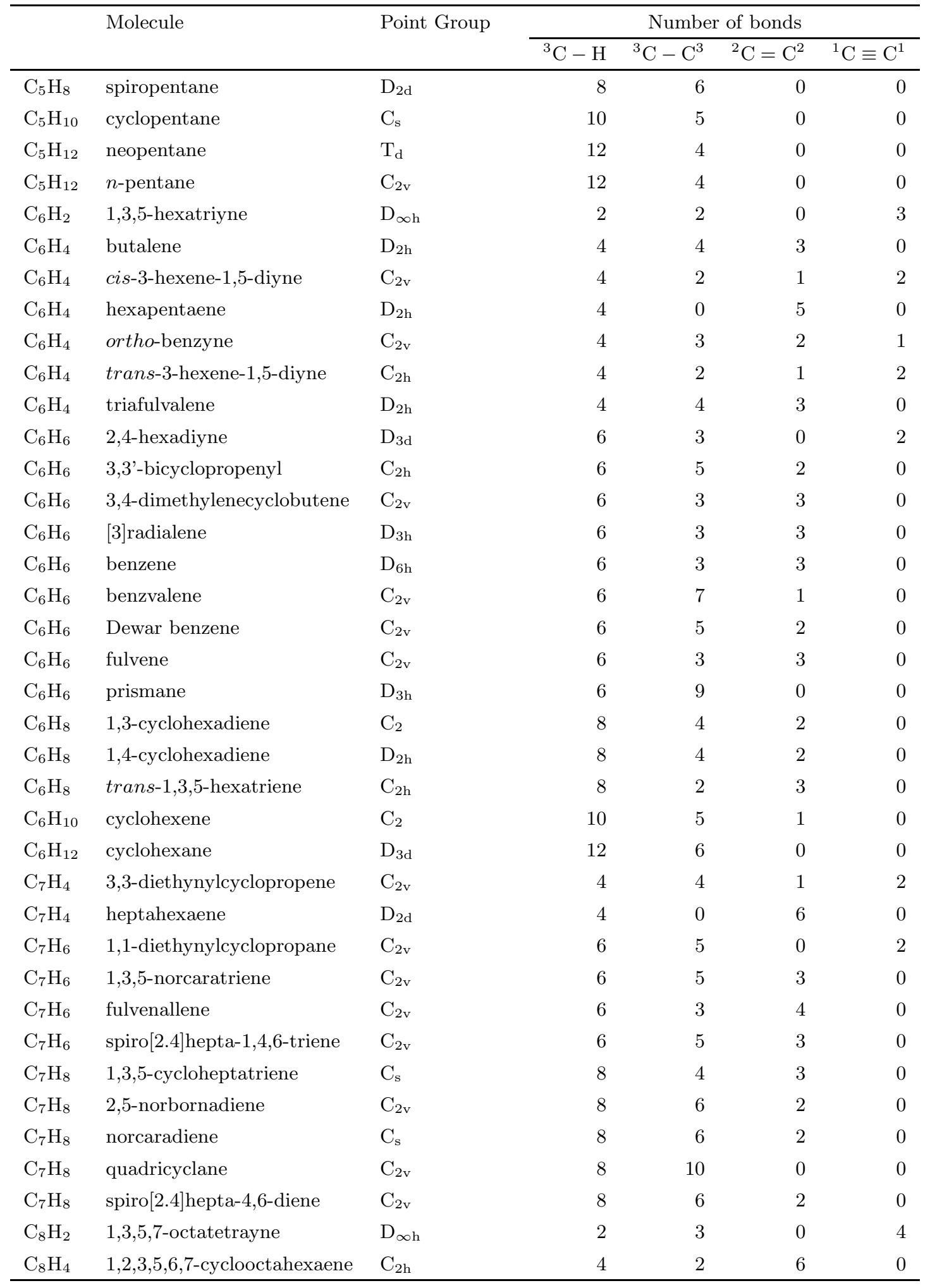


Table S1, contd.

\begin{tabular}{lllrrrr}
\hline & Molecule & Point Group & \multicolumn{4}{c}{ Number of bonds } \\
\cline { 4 - 7 } & & 4 & 4 & 2 & 2 \\
\hline $\mathrm{C}_{8} \mathrm{H}_{4}$ & 1,5-cyclooctadiene-3,7-diyne & $\mathrm{D}_{2 \mathrm{~h}}$ & 4 & 0 & 7 & 0 \\
$\mathrm{C}_{8} \mathrm{H}_{4}$ & octaheptaene & $\mathrm{D}_{2 \mathrm{~h}}$ & 6 & 5 & 4 & 0 \\
$\mathrm{C}_{8} \mathrm{H}_{6}$ & benzocyclobutadiene & $\mathrm{C}_{2 \mathrm{v}}$ & 6 & 7 & 3 & 0 \\
$\mathrm{C}_{8} \mathrm{H}_{6}$ & bis(cyclopropa)benzene & $\mathrm{D}_{2 \mathrm{~h}}$ & 6 & 5 & 4 & 0 \\
$\mathrm{C}_{8} \mathrm{H}_{6}$ & pentalene & $\mathrm{C}_{2 \mathrm{~h}}$ & 8 & 4 & 4 & 0 \\
$\mathrm{C}_{8} \mathrm{H}_{8}$ & $1,3,5,7$-cyclooctatetraene & $\mathrm{D}_{2 \mathrm{~d}}$ & 8 & 6 & 3 & 0 \\
$\mathrm{C}_{8} \mathrm{H}_{8}$ & benzocyclobutene & $\mathrm{C}_{2 \mathrm{v}}$ & 8 & 12 & 0 & 0 \\
$\mathrm{C}_{8} \mathrm{H}_{8}$ & cubane & $\mathrm{O}_{\mathrm{h}}$ & 8 & 4 & 4 & 0 \\
$\mathrm{C}_{8} \mathrm{H}_{8}$ & para-xylylene & $\mathrm{D}_{2 \mathrm{~h}}$ & 4 & 4 & 1 & 4 \\
$\mathrm{C}_{10} \mathrm{H}_{4}$ & tetraethynylethene & $\mathrm{D}_{2 \mathrm{~h}}$ & 8 & 6 & 5 & 0 \\
$\mathrm{C}_{10} \mathrm{H}_{8}$ & naphthalene & $\mathrm{D}_{2 \mathrm{~h}}$ & 8 & 6 & 5 & 0 \\
$\mathrm{C}_{10} \mathrm{H}_{8}$ & pentafulvalene & $\mathrm{D}_{2 \mathrm{~h}}$ & & & \\
\hline
\end{tabular}


Figure S1: Set of molecules, part 1 
<smiles>CCC</smiles>

propane

$\mathrm{H}_{2} \mathrm{C}=\mathrm{C}=\mathrm{C}=\mathrm{CH}_{2}$

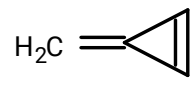

methylenecyclopropene
$\mathrm{HC} \equiv \quad \equiv \mathrm{CH}_{\mathrm{H}}$
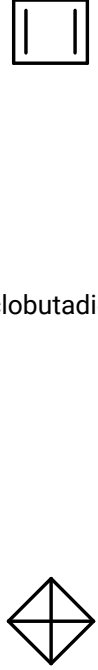

tetrahedrane bicyclo[1.1.0]but-1(3)-ene

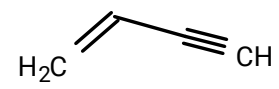

$\mathrm{H}_{2} \mathrm{C}=\mathrm{C} \stackrel{\stackrel{\mathrm{H}}{\mathrm{C}} \backslash}{\mathrm{CH}_{3}}$

Figure S2: Set of molecules, part 2 1,2-butadiene

ethynylethene

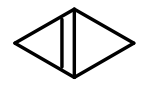




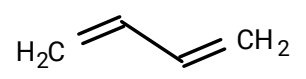

1,3-butadiene

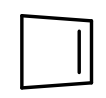

cyclobutene

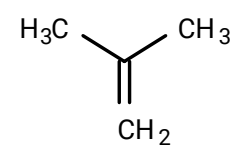

isobutene
$\mathrm{H}_{3} \mathrm{C}=\mathrm{CH}_{3}$

2-butyne

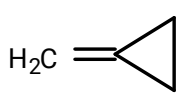

methylenecyclopropane

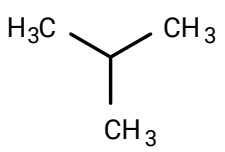

isobutane

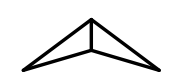

bicyclo[1.1.0]butane

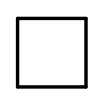

cyclobutane

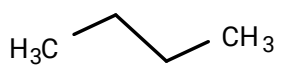

n-butane

Figure S3: Set of molecules, part 3 
$\mathrm{HC}_{\mathrm{HH}}$

1,4-pentadiyne

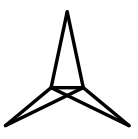

[1.1.1]propellane

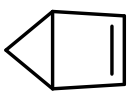

bicyclo[2.1.0]pent-2-ene
$\mathrm{H}_{2} \mathrm{C}=\mathrm{C}=\mathrm{C}=\mathrm{C}=\mathrm{CH}_{2}$

pentatetraene

spiropentadiene

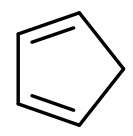

1,3-cyclopentadiene

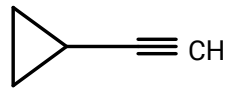

cyclopropylacetylene

1,4-pentadiene

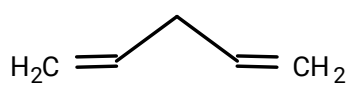

Figure S4: Set of molecules, part 4 
$\mathrm{H}_{2} \mathrm{C}$

isoprene

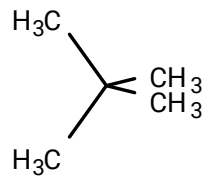

neopentane

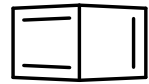

butalene

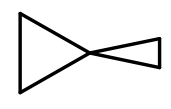

spiropentane

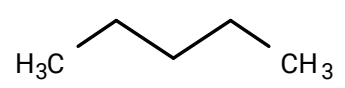

n-pentane

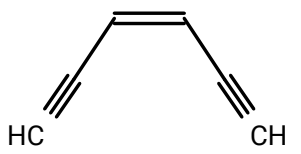

cis-3-hexene-1,5-diyne cyclopentane

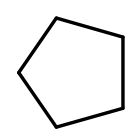

$\mathrm{HC} \equiv \quad \rightleftharpoons \mathrm{CH}$

1,3,5-hexatriyne

$\mathrm{H}_{2} \mathrm{C}=\mathrm{C}=\mathrm{C}=\mathrm{C}=\mathrm{C}=\mathrm{CH}_{2}$

Figure S5: Set of molecules, part 5 


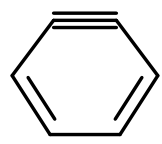

ortho-benzyne

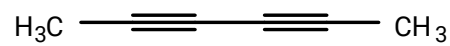

2,4-hexadiyne

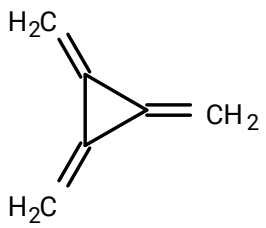

[3]radialene

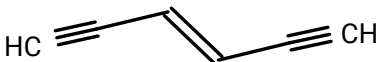

trans-3-hexene-1,5-diyne

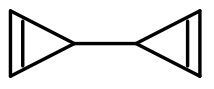

3,3'-bicyclopropenyl

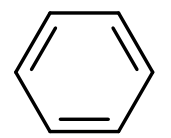

benzene

benzvalene

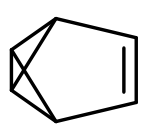

Figure S6: Set of molecules, part 6 

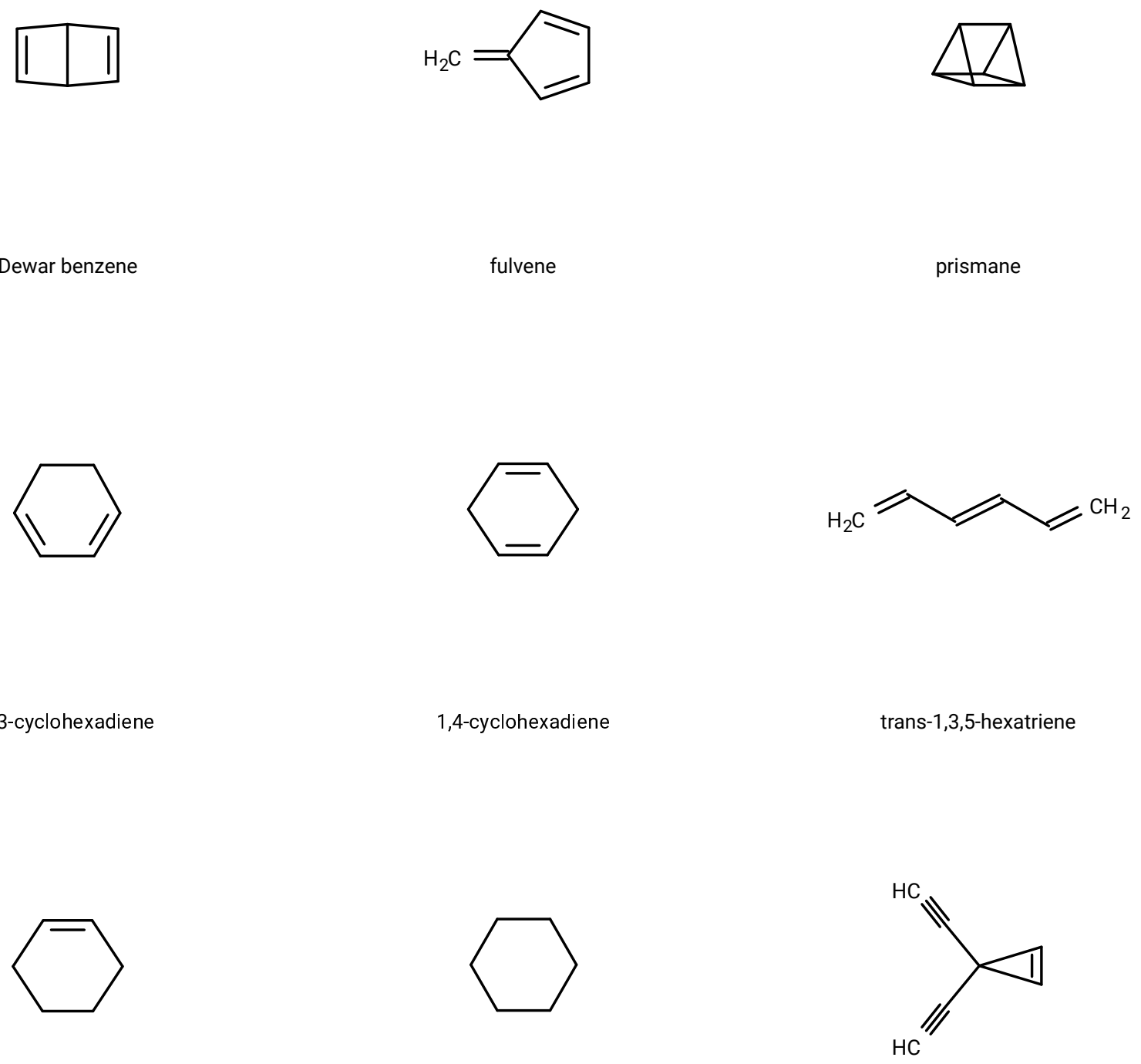

cyclohexene

cyclohexane

3,3-diethynylcyclopropene

Figure S7: Set of molecules, part 7 
$\mathrm{H}_{2} \mathrm{C}=\mathrm{c}=\mathrm{C}=\mathrm{C}=\mathrm{c}=\mathrm{c}=\mathrm{CH}_{2}$

heptahexaene

$\mathrm{H}_{2} \mathrm{C}=\mathrm{C}=\mathrm{C}_{\mathrm{C}}^{\mathrm{C}}=\stackrel{\mathrm{C}}{\mathrm{C}}=\stackrel{\mathrm{CH}}{\mathrm{C}}$

fulvenallene

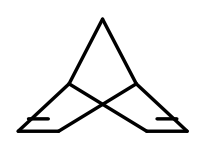

2,5-norbornadiene
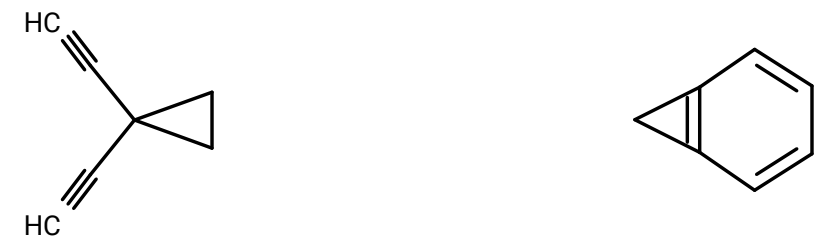

1,1-diethynylcyclopropane

1,3,5-norcaratriene

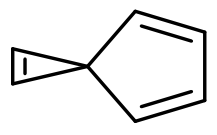

spiro[2.4]hepta-1,4,6-triene

1,3,5-cycloheptatriene
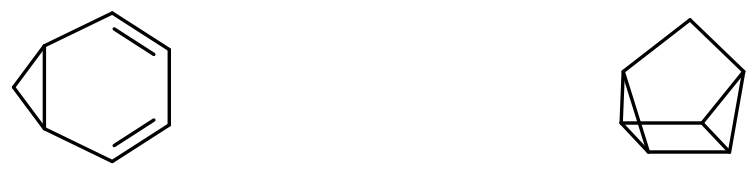

norcaradiene

quadricyclane

Figure S8: Set of molecules, part 8 


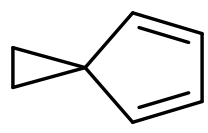

spiro[2.4]hepta-4,6-diene
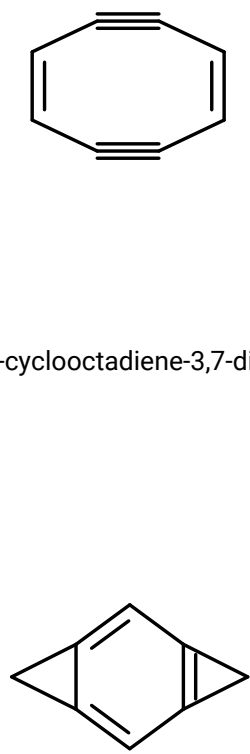

bis(cyclopropa)benzene
$\mathrm{HC}$

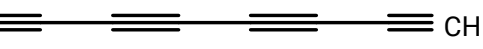

1,3,5,7-octatetrayne

$\mathrm{H}_{2} \mathrm{c}=\mathrm{c}=\mathrm{c}=\mathrm{c}=\mathrm{c}=\mathrm{c}=\mathrm{c}=\mathrm{CH}_{2}$

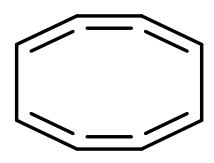

1,2,3,5,6,7-cyclooctahexaene

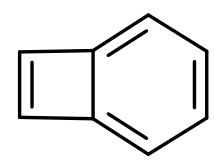

benzocyclobutadiene

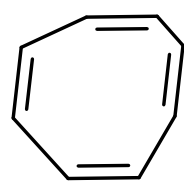

1,3,5,7-cyclooctatetraene

Figure S9: Set of molecules, part 9 


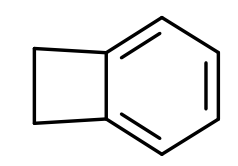

benzocyclobutene

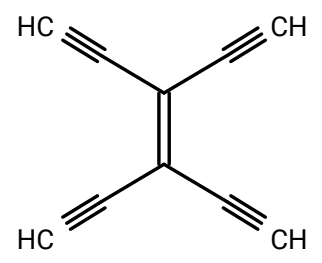

tetraethynylethene

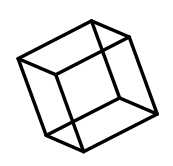

cubane

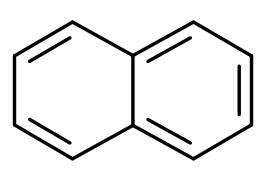

naphthalene

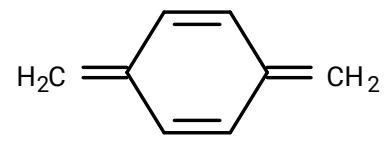

para-xylylene

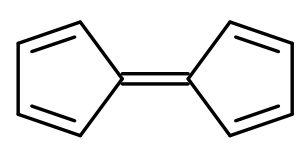

pentafulvalene

Figure S10: Set of molecules, part 10 
Table S2: CCSD valence-shell correlation contributions to the atomization energy at the complete basis set limit $(\mathrm{kcal} / \mathrm{mol})^{a}$

\begin{tabular}{llrrrrr}
\hline & Molecule & V5Z-F12 & V5Z-F12 & V5Z-F12rev2 & V5Z-F12rev2 & $\begin{array}{r}\text { V5Z-F12rev2 } \\
(1.2)\end{array}$ \\
& & $(1.4)$ & $(1.2)$ & $(1.4)$ & $(1.4) \Delta(\mathrm{F} 12 *)^{b}$ \\
\hline $\mathrm{CH}_{4}$ & methane & 84.545 & 84.577 & 84.595 & 84.609 & $\Delta_{\mathrm{C}}-0.047$ \\
$\mathrm{C}_{2} \mathrm{H}_{2}$ & acetylene & 94.893 & 94.925 & 94.919 & 94.941 & $2 \Delta_{\mathrm{C}}-0.113$ \\
$\mathrm{C}_{2} \mathrm{H}_{4}$ & ethylene & 119.093 & 119.134 & 119.146 & 119.166 & $2 \Delta_{\mathrm{C}}-0.100$ \\
$\mathrm{C}_{2} \mathrm{H}_{6}$ & ethane & 146.080 & 146.132 & 146.156 & 146.179 & $2 \Delta_{\mathrm{C}}-0.091$ \\
$\mathrm{C}_{3} \mathrm{H}_{4}$ & allene & 155.345 & 155.393 & 155.395 & 155.424 & $3 \Delta_{\mathrm{C}}-0.157$ \\
$\mathrm{C}_{3} \mathrm{H}_{4}$ & cyclopropene & 158.948 & 158.998 & 159.000 & 159.031 & $3 \Delta_{\mathrm{C}}-0.148$ \\
$\mathrm{C}_{3} \mathrm{H}_{4}$ & propyne & 155.907 & 155.958 & 155.958 & 155.990 & $3 \Delta_{\mathrm{C}}-0.154$ \\
$\mathrm{C}_{3} \mathrm{H}_{6}$ & cyclopropane & 183.932 & 183.991 & 184.008 & 184.038 & $3 \Delta_{\mathrm{C}}-0.136$ \\
$\mathrm{C}_{3} \mathrm{H}_{6}$ & propene & 181.392 & 181.451 & 181.467 & 181.497 & $3 \Delta_{\mathrm{C}}-0.144$ \\
$\mathrm{C}_{3} \mathrm{H}_{8}$ & propane & 208.656 & 208.726 & 208.756 & 208.788 & $3 \Delta_{\mathrm{C}}-0.137$ \\
$\mathrm{C}_{4} \mathrm{H}_{2}$ & 1,3-butadiyne & 168.084 & 168.134 & 168.110 & 168.150 & $4 \Delta_{\mathrm{C}}-0.227$ \\
$\mathrm{C}_{4} \mathrm{H}_{4}$ & bicyclo[1.1.0]but-1(3)-ene & 204.436 & 204.492 & 204.489 & 204.525 & $4 \Delta_{\mathrm{C}}-0.220$ \\
$\mathrm{C}_{4} \mathrm{H}_{4}$ & butatriene & 192.960 & 193.017 & 193.010 & 193.047 & $4 \Delta_{\mathrm{C}}-0.224$ \\
$\mathrm{C}_{4} \mathrm{H}_{4}$ & cyclobutadiene & 199.397 & 199.456 & 199.448 & 199.487 & $4 \Delta_{\mathrm{C}}-0.224$ \\
$\mathrm{C}_{4} \mathrm{H}_{4}$ & methylenecyclopropene & 191.794 & 191.852 & 191.846 & 191.884 & $4 \Delta_{\mathrm{C}}-0.193$ \\
$\mathrm{C}_{4} \mathrm{H}_{4}$ & tetrahedrane & 201.248 & 201.308 & 201.298 & 201.339 & $4 \Delta_{\mathrm{C}}-0.187$ \\
\hline
\end{tabular}

${ }^{a}$ Estimated from CCSD-F12b calculations (see Sec. 3). Geminal exponents are given in parenthesis.

${ }^{b}$ The last column lists the difference $\Delta\left(\mathrm{F} 12^{*}\right)$ between $\operatorname{CCSD}\left(\mathrm{F} 12^{*}\right)$ and CCSD-F12b. Unfortunately the program used (MOLPRO) lacks an open-shell implementation for $\operatorname{CCSD}\left(\mathrm{F} 12^{*}\right)$, hence the corresponding difference $\Delta_{\mathrm{C}}$ for the carbon atom can only be estimated using TURBOMOLE (version 7.1) and either a UHF-based CCSD scheme $\left(\Delta_{\mathrm{C}}=0.0474 \mathrm{kcal} / \mathrm{mol}\right)$ or an ROHF-based CCSD scheme $\left(\Delta_{\mathrm{C}}=0.0514 \mathrm{kcal} / \mathrm{mol}\right)$ with a semicanonicalization procedure different from that used by MOLPRO. The latter of the two values probably comes closest to the unavailable $\Delta_{\mathrm{C}}$ that is consistent with the MOLPRO implementation used for all CCSD and CCSD-F12b calculations. It affords shifts $\Delta\left(\mathrm{F} 12^{*}\right)$ in complete basis set estimates of correlation energy contributions to atomization energies (CCSD-F12b $\rightarrow$ CCSD-(F12*)) that range from $-0.021 \mathrm{kcal} / \mathrm{mol}$ (1,3-butadiyne) to $+0.019 \mathrm{kcal} / \mathrm{mol}$ (tetrahedrane). Note that these shifts are smaller than the differences observed between CCSD-F12b calculations using different versions of the V5Z-F12 basis set or different geminal exponents. 
Table S3: CCSD valence-shell correlation contributions to the atomization energy ( $\mathrm{kcal} / \mathrm{mol})$

\begin{tabular}{|c|c|c|c|c|c|c|c|c|c|}
\hline & Molecule & VTZ & VQZ & V5Z & V6Z & CVTZ & CVQZ & CV5Z & CV6Z \\
\hline $\mathrm{CH}_{4}$ & methane & 79.411 & 82.854 & 83.790 & 84.182 & 79.246 & 82.783 & 83.772 & 84.174 \\
\hline $\mathrm{C}_{2} \mathrm{H}_{2}$ & acetylene & 87.214 & 91.998 & 93.507 & 94.150 & 87.065 & 91.925 & 93.506 & 94.146 \\
\hline $\mathrm{C}_{2} \mathrm{H}_{4}$ & ethyl & 10.600 & 6.037 & 17.655 & 118.352 & 110.391 & 115.942 & 117.641 & 118.345 \\
\hline $\mathrm{C}_{2} \mathrm{H}_{6}$ & ethane & 136.393 & 142.716 & 144.545 & 145.317 & 136.069 & 142.581 & 144.512 & 145.303 \\
\hline $\mathrm{C}_{3} \mathrm{H}_{4}$ & allene & 43.331 & 150.962 & 153.227 & 154.213 & 143.107 & 150.820 & 153.220 & 154.208 \\
\hline $\mathrm{C}_{3} \mathrm{H}_{4}$ & cyclop & 146.458 & 154.277 & 156.735 & 157.773 & 146.123 & 154.141 & 156.717 & 157.764 \\
\hline $\mathrm{C}_{3} \mathrm{H}_{4}$ & propyne & 143.636 & 151.396 & 153.734 & 154.753 & 143.388 & 151.253 & 153.726 & 154.746 \\
\hline $\mathrm{C}_{3} \mathrm{H}_{6}$ & cyclopro1 & 170.374 & 178.929 & 181.600 & 182.725 & 169.946 & 178.780 & 181.569 & 182.711 \\
\hline $\mathrm{C}_{3} \mathrm{H}_{6}$ & propene & 168.208 & 176.642 & 179.151 & 180.230 & 167.866 & 176.483 & 179.124 & 180.218 \\
\hline $\mathrm{C}_{3} \mathrm{H}_{8}$ & propane & 194.354 & 203.617 & 206.335 & 207.485 & 193.884 & 203.418 & 206.287 & 207.465 \\
\hline $\mathrm{C}_{4} \mathrm{H}_{2}$ & 1,3-butadiyne & 153.532 & 162.530 & 165.338 & 166.571 & 153.211 & 162.357 & 165.344 & 166.566 \\
\hline $\mathrm{C}_{4} \mathrm{H}_{4}$ & bicyclo[1.1.0]but-1(3)-ene & 188.666 & 198.330 & 201.517 & 202.855 & 188.240 & 198.162 & 201.485 & 202.849 \\
\hline $\mathrm{C}_{4} \mathrm{H}_{4}$ & butatriene & 177.588 & 187.287 & 190.168 & 191.441 & 177.303 & 187.096 & 190.164 & 191.435 \\
\hline $\mathrm{C}_{4} \mathrm{H}_{4}$ & cyclobutadiene & 183.530 & 193.359 & 196.472 & 197.817 & 183.114 & 193.194 & 196.454 & 197.808 \\
\hline $\mathrm{C}_{4} \mathrm{H}_{4}$ & methylenecyclopropene & 175.655 & 185.720 & 188.866 & 190.213 & 175.316 & 185.560 & 188.859 & 190.206 \\
\hline $\mathrm{C}_{4} \mathrm{H}_{4}$ & tetrahedrane & 184.019 & 194.599 & 198.087 & 199.553 & 183.464 & 194.444 & 198.067 & 199.542 \\
\hline
\end{tabular}


Table S4: All-electron correlation contributions to the atomization energy (kcal/mol)

\begin{tabular}{|c|c|c|c|c|c|c|c|c|c|}
\hline & \multirow[b]{2}{*}{ Molecule } & \multicolumn{4}{|c|}{ CCSD(full) } & \multicolumn{4}{|c|}{$\operatorname{CCSD}(\mathrm{T})($ full $)$} \\
\hline & & CVTZ & CVQZ & CV5Z & CV6Z & CVTZ & CVQZ & CV5Z & CV6Z \\
\hline $\mathrm{CH}_{4}$ & methane & 80.258 & 83.948 & 84.984 & 85.401 & 82.868 & 86.753 & 87.839 & 88.275 \\
\hline $\mathrm{C}_{2} \mathrm{H}_{2}$ & acetylene & 88.771 & 93.982 & 95.671 & 96.346 & 96.683 & 102.330 & 104.150 & 104.874 \\
\hline $\mathrm{C}_{2} \mathrm{H}_{4}$ & ethylene & 112.176 & 118.018 & 119.811 & 120.546 & 119.018 & 125.280 & 127.198 & 127.980 \\
\hline $\mathrm{C}_{2} \mathrm{H}_{6}$ & ethane & 138.016 & 144.791 & 146.811 & 147.631 & 143.833 & 150.994 & 153.125 & 153.986 \\
\hline $\mathrm{C}_{3} \mathrm{H}_{4}$ & allene & 145.829 & 153.987 & 156.533 & 157.570 & 157.207 & 166.029 & 168.780 & 169.890 \\
\hline $\mathrm{C}_{3} \mathrm{H}_{4}$ & cyclopropene & 148.835 & 157.271 & 159.986 & 161.078 & 160.267 & 169.348 & 172.266 & 173.431 \\
\hline $\mathrm{C}_{3} \mathrm{H}_{4}$ & propyne & 146.141 & 154.476 & 157.100 & 158.170 & 157.336 & 166.313 & 169.133 & 170.273 \\
\hline $\mathrm{C}_{3} \mathrm{H}_{6}$ & cyclopropane & 172.874 & 182.104 & 185.030 & 186.216 & 182.814 & 192.669 & 195.788 & 197.044 \\
\hline $\mathrm{C}_{3} \mathrm{H}_{6}$ & propene & 170.650 & 179.677 & 182.455 & 183.595 & 180.938 & 190.594 & 193.562 & 194.771 \\
\hline $\mathrm{C}_{3} \mathrm{H}_{8}$ & propane & 196.783 & 206.691 & 209.689 & 210.910 & 206.030 & 216.534 & 219.708 & 220.993 \\
\hline $\mathrm{C}_{4} \mathrm{H}_{2}$ & 1,3-butadiyne & 156.741 & 166.544 & 169.743 & 171.033 & 174.547 & 185.252 & 188.727 & 190.120 \\
\hline $\mathrm{C}_{4} \mathrm{H}_{4}$ & bicyclo[1.1.0]but-1(3)-ene & 191.392 & 201.694 & 205.162 & 206.571 & 210.363 & 221.560 & 225.318 & 226.834 \\
\hline $\mathrm{C}_{4} \mathrm{H}_{4}$ & butatriene & 180.894 & 191.268 & 194.530 & 195.865 & 197.977 & 209.256 & 212.797 & 214.235 \\
\hline $\mathrm{C}_{4} \mathrm{H}_{4}$ & cyclobutadiene & 186.397 & 196.954 & 200.380 & 201.789 & 203.720 & 215.178 & 218.891 & 220.407 \\
\hline $\mathrm{C}_{4} \mathrm{H}_{4}$ & methylenecyclopropene & 178.962 & 189.768 & 193.256 & 194.665 & 194.816 & 206.485 & 210.248 & 211.756 \\
\hline $\mathrm{C}_{4} \mathrm{H}_{4}$ & tetrahedrane & 187.369 & 198.882 & 202.690 & 204.222 & 203.029 & 215.512 & 219.632 & 221.274 \\
\hline
\end{tabular}


Table S5: Effective extrapolation exponents for CCSD/cc-pVXZ valence shell correlation contributions to atomization energies ${ }^{a}$

\begin{tabular}{llrrrrrr}
\hline & Molecule & $(34) \rightarrow 5$ & $(34) \rightarrow 6$ & $(34) \rightarrow \infty$ & $(45) \rightarrow 6$ & $(45) \rightarrow \infty$ & $(56) \rightarrow \infty$ \\
\hline $\mathrm{CH}_{4}$ & methane & 4.02 & 3.88 & 3.77 & 3.26 & 3.42 & 3.57 \\
$\mathrm{C}_{2} \mathrm{H}_{2}$ & acetylene & 3.46 & 3.41 & 3.36 & 3.18 & 3.22 & 3.26 \\
$\mathrm{C}_{2} \mathrm{H}_{4}$ & ethylene & 3.68 & 3.58 & 3.50 & 3.12 & 3.26 & 3.39 \\
$\mathrm{C}_{2} \mathrm{H}_{6}$ & ethane & 3.79 & 3.68 & 3.61 & 3.22 & 3.37 & 3.51 \\
$\mathrm{C}_{3} \mathrm{H}_{4}$ & allene & 3.69 & 3.57 & 3.47 & 3.07 & 3.18 & 3.27 \\
$\mathrm{C}_{3} \mathrm{H}_{4}$ & cyclopropene & 3.47 & 3.43 & 3.38 & 3.22 & 3.26 & 3.30 \\
$\mathrm{C}_{3} \mathrm{H}_{4}$ & propyne & 3.63 & 3.53 & 3.44 & 3.07 & 3.19 & 3.30 \\
$\mathrm{C}_{3} \mathrm{H}_{6}$ & cyclopropane & 3.50 & 3.45 & 3.42 & 3.23 & 3.32 & 3.39 \\
$\mathrm{C}_{3} \mathrm{H}_{6}$ & propene & 3.68 & 3.58 & 3.50 & 3.13 & 3.26 & 3.38 \\
$\mathrm{C}_{3} \mathrm{H}_{8}$ & propane & 3.73 & 3.64 & 3.57 & 3.21 & 3.34 & 3.47 \\
$\mathrm{C}_{4} \mathrm{H}_{2}$ & 1,3-butadiyne & 3.50 & 3.41 & 3.32 & 3.03 & 3.10 & 3.17 \\
$\mathrm{C}_{4} \mathrm{H}_{4}$ & bicyclo[1.1.0]but-1(3)-ene & 3.29 & 3.28 & 3.27 & 3.25 & 3.24 & 3.23 \\
$\mathrm{C}_{4} \mathrm{H}_{4}$ & butatriene & 3.69 & 3.56 & 3.43 & 3.00 & 3.11 & 3.20 \\
$\mathrm{C}_{4} \mathrm{H}_{4}$ & cyclobutadiene & 3.44 & 3.38 & 3.33 & 3.11 & 3.18 & 3.24 \\
$\mathrm{C}_{4} \mathrm{H}_{4}$ & methylenecyclopropene & 3.49 & 3.43 & 3.37 & 3.15 & 3.20 & 3.24 \\
$\mathrm{C}_{4} \mathrm{H}_{4}$ & tetrahedrane & 3.29 & 3.28 & 3.28 & 3.24 & 3.27 & 3.29 \\
\hline
\end{tabular}

${ }^{a}$ CCSD-F12b/cc-pV5Z-F12rev2 with a geminal exponent of 1.4 is used as complete-basis-set reference. 
Table S6: Effective extrapolation exponents for CCSD/cc-pCVXZ valence shell correlation contributions to atomization energies ${ }^{a}$

\begin{tabular}{llrrrrrr}
\hline & Molecule & $(34) \rightarrow 5$ & $(34) \rightarrow 6$ & $(34) \rightarrow \infty$ & $(45) \rightarrow 6$ & $(45) \rightarrow \infty$ & $(56) \rightarrow \infty$ \\
\hline $\mathrm{CH}_{4}$ & methane & 3.91 & 3.82 & 3.75 & 3.40 & 3.50 & 3.59 \\
$\mathrm{C}_{2} \mathrm{H}_{2}$ & acetylene & 3.34 & 3.36 & 3.34 & 3.42 & 3.33 & 3.24 \\
$\mathrm{C}_{2} \mathrm{H}_{4}$ & ethylene & 3.57 & 3.52 & 3.48 & 3.31 & 3.35 & 3.40 \\
$\mathrm{C}_{2} \mathrm{H}_{6}$ & ethane & 3.69 & 3.63 & 3.59 & 3.37 & 3.45 & 3.53 \\
$\mathrm{C}_{3} \mathrm{H}_{4}$ & allene & 3.51 & 3.48 & 3.42 & 3.34 & 3.30 & 3.26 \\
$\mathrm{C}_{3} \mathrm{H}_{4}$ & cyclopropene & 3.39 & 3.39 & 3.37 & 3.41 & 3.35 & 3.30 \\
$\mathrm{C}_{3} \mathrm{H}_{4}$ & propyne & 3.47 & 3.45 & 3.40 & 3.33 & 3.31 & 3.28 \\
$\mathrm{C}_{3} \mathrm{H}_{6}$ & cyclopropane & 3.46 & 3.44 & 3.43 & 3.37 & 3.39 & 3.41 \\
$\mathrm{C}_{3} \mathrm{H}_{6}$ & propene & 3.57 & 3.52 & 3.48 & 3.31 & 3.35 & 3.39 \\
$\mathrm{C}_{3} \mathrm{H}_{8}$ & propane & 3.64 & 3.59 & 3.55 & 3.36 & 3.42 & 3.49 \\
$\mathrm{C}_{4} \mathrm{H}_{2}$ & 1,3-butadiyne & 3.33 & 3.34 & 3.29 & 3.37 & 3.25 & 3.14 \\
$\mathrm{C}_{4} \mathrm{H}_{4}$ & bicyclo[1.1.0]but-1(3)-ene & 3.23 & 3.26 & 3.27 & 3.36 & 3.31 & 3.27 \\
$\mathrm{C}_{4} \mathrm{H}_{4}$ & butatriene & 3.49 & 3.45 & 3.38 & 3.31 & 3.25 & 3.19 \\
$\mathrm{C}_{4} \mathrm{H}_{4}$ & cyclobutadiene & 3.36 & 3.35 & 3.32 & 3.30 & 3.27 & 3.24 \\
$\mathrm{C}_{4} \mathrm{H}_{4}$ & methylenecyclopropene & 3.38 & 3.38 & 3.35 & 3.38 & 3.30 & 3.23 \\
$\mathrm{C}_{4} \mathrm{H}_{4}$ & tetrahedrane & 3.29 & 3.31 & 3.31 & 3.40 & 3.34 & 3.29 \\
\hline
\end{tabular}

${ }^{a}$ CCSD-F12b/cc-pV5Z-F12rev2 with a geminal exponent of 1.4 is used as complete-basis-set reference. 
Table S7: Effective extrapolation exponents for CCSD(full)/cc-pCVXZ correlation contributions to atomization energies

\begin{tabular}{llrrr}
\hline & Molecule & $(34) \rightarrow 5$ & $(34) \rightarrow 6$ & $(45) \rightarrow 6$ \\
\hline $\mathrm{CH}_{4}$ & methane & 3.90 & 3.82 & 3.45 \\
$\mathrm{C}_{2} \mathrm{H}_{2}$ & acetylene & 3.36 & 3.38 & 3.49 \\
$\mathrm{C}_{2} \mathrm{H}_{4}$ & ethylene & 3.56 & 3.53 & 3.36 \\
$\mathrm{C}_{2} \mathrm{H}_{6}$ & ethane & 3.67 & 3.62 & 3.41 \\
$\mathrm{C}_{3} \mathrm{H}_{4}$ & allene & 3.50 & 3.48 & 3.39 \\
$\mathrm{C}_{3} \mathrm{H}_{4}$ & cyclopropene & 3.38 & 3.40 & 3.46 \\
$\mathrm{C}_{3} \mathrm{H}_{4}$ & propyne & 3.47 & 3.45 & 3.39 \\
$\mathrm{C}_{3} \mathrm{H}_{6}$ & cyclopropane & 3.44 & 3.44 & 3.42 \\
$\mathrm{C}_{3} \mathrm{H}_{6}$ & propene & 3.55 & 3.52 & 3.36 \\
$\mathrm{C}_{3} \mathrm{H}_{8}$ & propane & 3.62 & 3.58 & 3.40 \\
$\mathrm{C}_{4} \mathrm{H}_{2}$ & 1,3-butadiyne & 3.33 & 3.35 & 3.44 \\
$\mathrm{C}_{4} \mathrm{H}_{4}$ & bicyclo[1.1.0]but-1(3)-ene & 3.21 & 3.25 & 3.41 \\
$\mathrm{C}_{4} \mathrm{H}_{4}$ & butatriene & 3.47 & 3.45 & 3.37 \\
$\mathrm{C}_{4} \mathrm{H}_{4}$ & cyclobutadiene & 3.35 & 3.35 & 3.35 \\
$\mathrm{C}_{4} \mathrm{H}_{4}$ & methylenecyclopropene & 3.37 & 3.38 & 3.43 \\
$\mathrm{C}_{4} \mathrm{H}_{4}$ & tetrahedrane & 3.28 & 3.31 & 3.45 \\
\hline
\end{tabular}


Table S8: Effective extrapolation exponents for $C C S D(T)($ full $) / c c-p C V X Z$ correlation contributions to atomization energies.

\begin{tabular}{llrrr}
\hline & Molecule & $(34) \rightarrow 5$ & $(34) \rightarrow 6$ & $(45) \rightarrow 6$ \\
\hline $\mathrm{CH}_{4}$ & methane & 3.91 & 3.83 & 3.46 \\
$\mathrm{C}_{2} \mathrm{H}_{2}$ & acetylene & 3.38 & 3.40 & 3.51 \\
$\mathrm{C}_{2} \mathrm{H}_{4}$ & ethylene & 3.57 & 3.54 & 3.39 \\
$\mathrm{C}_{2} \mathrm{H}_{6}$ & ethane & 3.68 & 3.63 & 3.43 \\
$\mathrm{C}_{3} \mathrm{H}_{4}$ & allene & 3.50 & 3.49 & 3.44 \\
$\mathrm{C}_{3} \mathrm{H}_{4}$ & cyclopropene & 3.39 & 3.41 & 3.49 \\
$\mathrm{C}_{3} \mathrm{H}_{4}$ & propyne & 3.47 & 3.47 & 3.43 \\
$\mathrm{C}_{3} \mathrm{H}_{6}$ & cyclopropane & 3.45 & 3.45 & 3.45 \\
$\mathrm{C}_{3} \mathrm{H}_{6}$ & propene & 3.56 & 3.53 & 3.39 \\
$\mathrm{C}_{3} \mathrm{H}_{8}$ & propane & 3.62 & 3.59 & 3.42 \\
$\mathrm{C}_{4} \mathrm{H}_{2}$ & 1,3-butadiyne & 3.35 & 3.37 & 3.47 \\
$\mathrm{C}_{4} \mathrm{H}_{4}$ & bicyclo[1.1.0]but-1(3)-ene & 3.22 & 3.26 & 3.44 \\
$\mathrm{C}_{4} \mathrm{H}_{4}$ & butatriene & 3.48 & 3.46 & 3.41 \\
$\mathrm{C}_{4} \mathrm{H}_{4}$ & cyclobutadiene & 3.36 & 3.36 & 3.38 \\
$\mathrm{C}_{4} \mathrm{H}_{4}$ & methylenecyclopropene & 3.38 & 3.39 & 3.47 \\
$\mathrm{C}_{4} \mathrm{H}_{4}$ & tetrahedrane & 3.29 & 3.33 & 3.50 \\
\hline
\end{tabular}


Table S9: $\operatorname{CCSD}(\mathrm{T})$ atomization energies $(\mathrm{kcal} / \mathrm{mol})^{a}$

\begin{tabular}{|c|c|c|c|c|}
\hline & Molecule & REF(hc) & model & difference \\
\hline $\mathrm{CH}_{4}$ & methane & 420.376 & 420.376 & $-{ }^{b}$ \\
\hline $\mathrm{C}_{2} \mathrm{H}_{2}$ & acetylene & 405.242 & 405.242 & $-b$ \\
\hline $\mathrm{C}_{2} \mathrm{H}_{4}$ & ethylene & 563.980 & 563.980 & $-b$ \\
\hline $\mathrm{C}_{2} \mathrm{H}_{6}$ & ethane & 713.087 & 713.087 & $-b$ \\
\hline $\mathrm{C}_{3} \mathrm{H}_{4}$ & allene & 703.939 & 704.013 & 0.074 \\
\hline $\mathrm{C}_{3} \mathrm{H}_{4}$ & cyclopropene & 681.984 & 682.042 & 0.058 \\
\hline $\mathrm{C}_{3} \mathrm{H}_{4}$ & propyne & 705.346 & 705.353 & 0.007 \\
\hline $\mathrm{C}_{3} \mathrm{H}_{6}$ & cyclopropane & 854.146 & 854.167 & 0.021 \\
\hline $\mathrm{C}_{3} \mathrm{H}_{6}$ & propene & 861.482 & 861.501 & 0.019 \\
\hline $\mathrm{C}_{3} \mathrm{H}_{8}$ & propane & 1007.927 & 1007.943 & 0.016 \\
\hline $\mathrm{C}_{4} \mathrm{H}_{2}$ & 1,3-butadiyne & 696.808 & 696.827 & 0.019 \\
\hline $\mathrm{C}_{4} \mathrm{H}_{4}$ & bicyclo[1.1.0]but-1(3)-ene & 787.141 & 787.120 & -0.020 \\
\hline $\mathrm{C}_{4} \mathrm{H}_{4}$ & butatriene & 845.506 & 845.611 & 0.104 \\
\hline $\mathrm{C}_{4} \mathrm{H}_{4}$ & cyclobutadiene & 820.378 & 820.383 & 0.005 \\
\hline $\mathrm{C}_{4} \mathrm{H}_{4}$ & ethynylethene & 853.869 & 853.895 & 0.027 \\
\hline $\mathrm{C}_{4} \mathrm{H}_{4}$ & methylenecyclopropene & 830.255 & 830.354 & 0.099 \\
\hline $\mathrm{C}_{4} \mathrm{H}_{4}$ & tetrahedrane & 793.970 & 794.069 & 0.099 \\
\hline $\mathrm{C}_{4} \mathrm{H}_{6}$ & 1,2-butadiene & 1000.412 & 1000.508 & 0.096 \\
\hline $\mathrm{C}_{4} \mathrm{H}_{6}$ & 1,3-butadiene & 1013.120 & 1013.154 & 0.034 \\
\hline $\mathrm{C}_{4} \mathrm{H}_{6}$ & 2-butyne & 1004.475 & 1004.496 & 0.021 \\
\hline $\mathrm{C}_{4} \mathrm{H}_{6}$ & bicyclo[1.1.0]butane & 986.764 & 986.776 & 0.012 \\
\hline $\mathrm{C}_{4} \mathrm{H}_{6}$ & cyclobutene & 1001.874 & 1001.928 & 0.055 \\
\hline $\mathrm{C}_{4} \mathrm{H}_{6}$ & methylenecyclopropane & 993.543 & 993.617 & 0.074 \\
\hline $\mathrm{C}_{4} \mathrm{H}_{8}$ & cyclobutane & 1150.718 & 1150.727 & 0.009 \\
\hline $\mathrm{C}_{4} \mathrm{H}_{8}$ & isobutene & 1159.728 & 1159.788 & 0.060 \\
\hline $\mathrm{C}_{5} \mathrm{H}_{4}$ & 1,4-pentadiyne & 987.651 & 987.672 & 0.021 \\
\hline $\mathrm{C}_{5} \mathrm{H}_{4}$ & pentatetraene & 988.600 & 988.749 & 0.149 \\
\hline $\mathrm{C}_{5} \mathrm{H}_{4}$ & spiropentadiene & 941.718 & 941.822 & 0.104 \\
\hline $\mathrm{C}_{6} \mathrm{H}_{2}$ & 1,3,5-hexatriyne & 989.516 & 989.498 & -0.018 \\
\hline $\mathrm{C}_{6} \mathrm{H}_{4}$ & butalene & 1101.408 & 1101.519 & 0.112 \\
\hline $\mathrm{C}_{6} \mathrm{H}_{4}$ & hexapentaene & 1132.074 & 1132.268 & 0.194 \\
\hline $\mathrm{C}_{6} \mathrm{H}_{4}$ & triafulvalene & 1084.627 & 1084.793 & 0.166 \\
\hline $\mathrm{C}_{6} \mathrm{H}_{6}$ & benzene & 1369.589 & 1369.683 & 0.094 \\
\hline
\end{tabular}

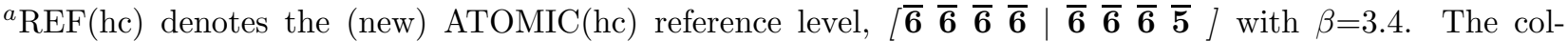
umn labeled "model" lists ATOMIC/A* values, corrected with bond terms $C_{A, e}^{\operatorname{CCSD}(\mathrm{T})\{H\}}[b]$ (Table 1) for the change in reference level toward $\operatorname{ATOMIC}(\mathrm{hc})$, reported energies are thus defined as $E_{A, e}^{\mathrm{CCSD}(\mathrm{T})\left\{\mathrm{A}^{*}\right\}}[M]+$ $\sum_{b \in M} C_{A, e}^{\mathrm{CCSD}(\mathrm{T})\{H\}}[b]$.

${ }^{b}$ The model recovers reference data for BSR prototypes (methane, ethane, ethylene, acetylene) by construction. These data are not included in Figure 1 or in the statistical analysis discussed in the text. 
Table S10: Higher order electron correlation contributions to atomization energies $(\mathrm{kcal} / \mathrm{mol} \text {, non-extrapolated })^{a}$

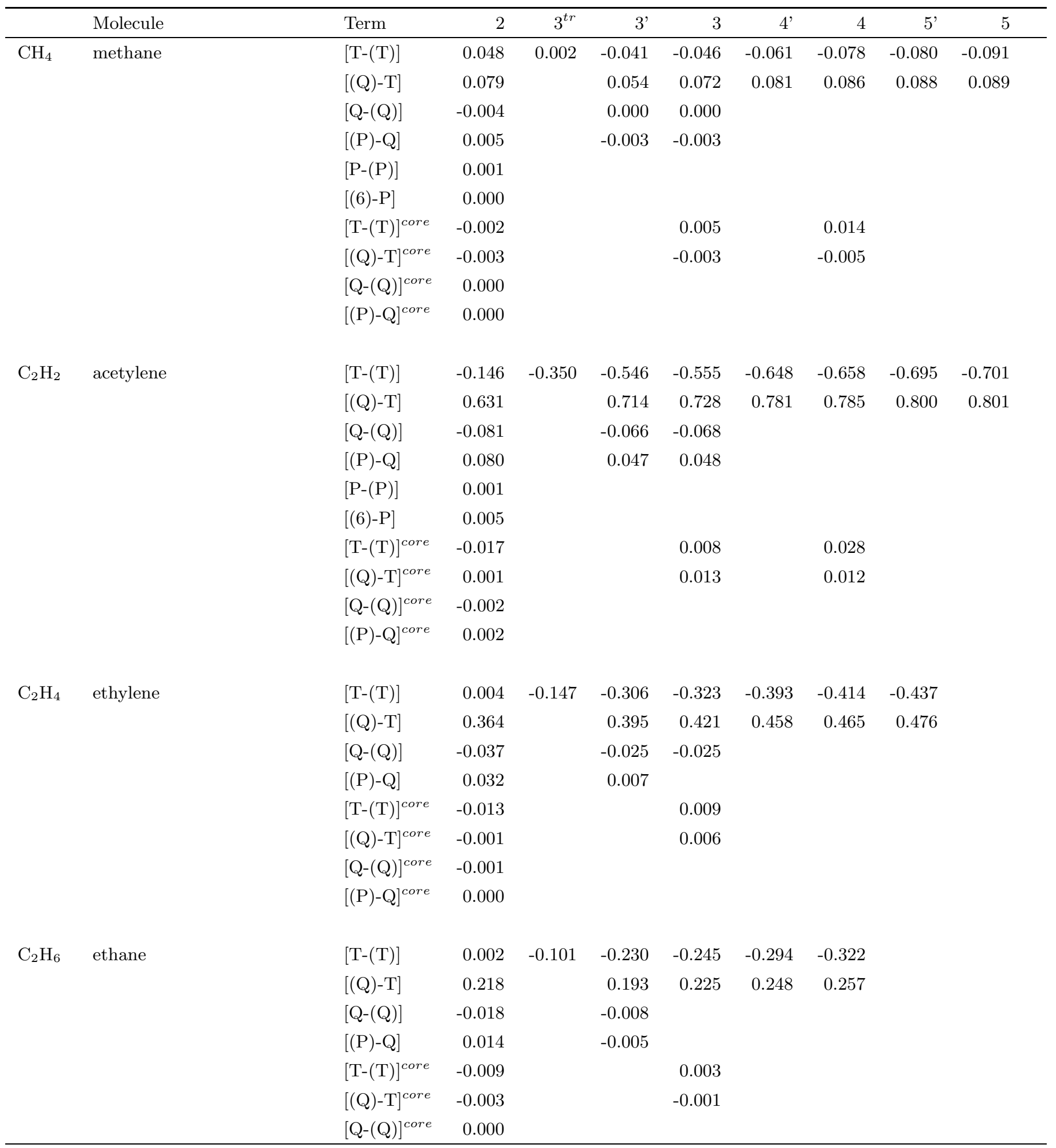


Table S10, contd.

\begin{tabular}{|c|c|c|c|c|c|c|c|c|c|c|}
\hline & Molecule & Term & 2 & $3^{t r}$ & $3^{\prime}$ & 3 & $4^{\prime}$ & 4 & 5 & 5 \\
\hline & & {$[(\mathrm{P})-\mathrm{Q}]^{\text {core }}$} & 0.000 & & & & & & & \\
\hline \multirow[t]{6}{*}{$\mathrm{C}_{3} \mathrm{H}_{4}$} & allene & {$[\mathrm{T}-(\mathrm{T})]$} & -0.070 & -0.315 & -0.594 & -0.618 & -0.744 & -0.768 & & \\
\hline & & {$[(\mathrm{Q})-\mathrm{T}]$} & 0.687 & & 0.790 & 0.820 & 0.892 & & & \\
\hline & & {$[\mathrm{Q}-(\mathrm{Q})]$} & -0.083 & & & & & & & \\
\hline & & {$[(\mathrm{P})-\mathrm{Q}]$} & 0.057 & & & & & & & \\
\hline & & {$[\mathrm{T}-(\mathrm{T})]^{\text {core }}$} & -0.021 & & & 0.012 & & & & \\
\hline & & {$[(\mathrm{Q})-\mathrm{T}]^{\text {core }}$} & 0.003 & & & 0.017 & & & & \\
\hline \multirow[t]{6}{*}{$\mathrm{C}_{3} \mathrm{H}_{4}$} & cyclopropene & {$[\mathrm{T}-(\mathrm{T})]$} & -0.287 & -0.538 & -0.836 & -0.860 & -0.991 & -1.014 & & \\
\hline & & {$[(\mathrm{Q})-\mathrm{T}]$} & 0.752 & & 0.837 & 0.867 & 0.938 & & & \\
\hline & & {$[\mathrm{Q}-(\mathrm{Q})]$} & -0.095 & & & & & & & \\
\hline & & {$[(\mathrm{P})-\mathrm{Q}]$} & 0.069 & & & & & & & \\
\hline & & {$[\mathrm{T}-(\mathrm{T})]^{c o r e}$} & -0.025 & & & 0.001 & & & & \\
\hline & & {$[(\mathrm{Q})-\mathrm{T}]^{\text {core }}$} & 0.004 & & & 0.019 & & & & \\
\hline \multirow[t]{5}{*}{$\mathrm{C}_{3} \mathrm{H}_{4}$} & propyne & {$[\mathrm{T}-(\mathrm{T})]$} & -0.240 & -0.492 & -0.773 & -0.791 & -0.915 & & & \\
\hline & & {$[(\mathrm{Q})-\mathrm{T}]$} & 0.798 & & 0.889 & 0.915 & 0.985 & & & \\
\hline & & {$[\mathrm{Q}-(\mathrm{Q})]$} & -0.112 & & & & & & & \\
\hline & & {$[\mathrm{T}-(\mathrm{T})]^{\text {core }}$} & -0.023 & & & 0.009 & & & & \\
\hline & & {$[(\mathrm{Q})-\mathrm{T}]^{\text {core }}$} & 0.001 & & & & & & & \\
\hline \multirow[t]{4}{*}{$\mathrm{C}_{3} \mathrm{H}_{6}$} & cyclopropane & {$[\mathrm{T}-(\mathrm{T})]$} & -0.204 & -0.392 & -0.649 & -0.677 & -0.782 & & & \\
\hline & & {$[(\mathrm{Q})-\mathrm{T}]$} & 0.497 & & 0.507 & 0.546 & 0.599 & & & \\
\hline & & {$[\mathrm{T}-(\mathrm{T})]^{\text {core }}$} & -0.021 & & & -0.003 & & & & \\
\hline & & {$[(\mathrm{Q})-\mathrm{T}]^{\text {core }}$} & 0.000 & & & & & & & \\
\hline \multirow[t]{4}{*}{$\mathrm{C}_{3} \mathrm{H}_{6}$} & propene & {$[\mathrm{T}-(\mathrm{T})]$} & -0.080 & -0.288 & -0.533 & -0.560 & & & & \\
\hline & & {$[(\mathrm{Q})-\mathrm{T}]$} & 0.538 & & 0.578 & & & & & \\
\hline & & {$[\mathrm{T}-(\mathrm{T})]^{\text {core }}$} & -0.020 & & & & & & & \\
\hline & & {$[(\mathrm{Q})-\mathrm{T}]^{\text {core }}$} & 0.000 & & & & & & & \\
\hline \multirow[t]{4}{*}{$\mathrm{C}_{3} \mathrm{H}_{8}$} & propane & {$[\mathrm{T}-(\mathrm{T})]$} & -0.066 & -0.227 & -0.444 & -0.470 & -0.554 & & & \\
\hline & & {$[(\mathrm{Q})-\mathrm{T}]$} & 0.371 & & 0.352 & & & & & \\
\hline & & {$[\mathrm{T}-(\mathrm{T})]^{\text {core }}$} & -0.017 & & & & & & & \\
\hline & & {$[(\mathrm{Q})-\mathrm{T}]^{\text {core }}$} & -0.003 & & & & & & & \\
\hline \multirow[t]{2}{*}{$\mathrm{C}_{4} \mathrm{H}_{2}$} & 1,3-butadiyne & {$[\mathrm{T}-(\mathrm{T})]$} & -0.587 & -0.997 & -1.460 & -1.471 & -1.681 & -1.692 & & \\
\hline & & {$[(\mathrm{Q})-\mathrm{T}]$} & 1.632 & & 1.898 & 1.912 & 2.043 & 2.047 & & \\
\hline
\end{tabular}


Table S10, contd.

\begin{tabular}{|c|c|c|c|c|c|c|c|c|c|c|}
\hline & Molecule & Term & 2 & $3^{t r}$ & $3^{\prime}$ & 3 & $4^{\prime}$ & 4 & $5^{\prime}$ & 5 \\
\hline & & {$[\mathrm{Q}-(\mathrm{Q})]$} & -0.248 & & & & & & & \\
\hline & & {$[(\mathrm{P})-\mathrm{Q}]$} & 0.183 & & & & & & & \\
\hline & & {$[(\mathrm{Q})-\mathrm{T}]^{\text {core }}$} & 0.009 & & & 0.037 & & & & \\
\hline \multirow{4}{*}{$\mathrm{C}_{4} \mathrm{H}_{4}$} & & {$[(\mathrm{Q})-\mathrm{T}]$} & 1.763 & & 1.984 & & & & & \\
\hline & & {$[\mathrm{Q}-(\mathrm{Q})]$} & -0.296 & & & & & & & \\
\hline & & {$[\mathrm{T}-(\mathrm{T})]^{\text {core }}$} & -0.037 & & & & & & & \\
\hline & & {$[(\mathrm{Q})-\mathrm{T}]^{\text {core }}$} & 0.019 & & & & & & & \\
\hline \multirow{3}{*}{$\mathrm{C}_{4} \mathrm{H}_{4}$} & & {$[\mathrm{Q}-(\mathrm{Q})]$} & -0.173 & & & & & & & \\
\hline & & {$[\mathrm{T}-(\mathrm{T})]^{\text {core }}$} & -0.030 & & & & & & & \\
\hline & & {$[(\mathrm{Q})-\mathrm{T}]^{\text {core }}$} & 0.011 & & & & & & & \\
\hline \multirow[t]{5}{*}{$\mathrm{C}_{4} \mathrm{H}_{4}$} & cyclobutadiene & {$[\mathrm{T}-(\mathrm{T})]$} & -0.150 & -0.550 & -0.996 & -1.025 & -1.231 & -1.256 & & \\
\hline & & {$[(\mathrm{Q})-\mathrm{T}]$} & 1.331 & & 1.554 & 1.590 & 1.705 & & & \\
\hline & & {$[\mathrm{Q}-(\mathrm{Q})]$} & -0.188 & & & & & & & \\
\hline & & {$[\mathrm{T}-(\mathrm{T})]^{\text {core }}$} & -0.035 & & & & & & & \\
\hline & & {$[(\mathrm{Q})-\mathrm{T}]^{\text {core }}$} & 0.015 & & & & & & & \\
\hline \multirow[t]{5}{*}{$\mathrm{C}_{4} \mathrm{H}_{4}$} & methylenecyclopropene & {$[\mathrm{T}-(\mathrm{T})]$} & -0.422 & -0.757 & -1.179 & -1.204 & & & & \\
\hline & & {$[(\mathrm{Q})-\mathrm{T}]$} & 1.060 & & 1.217 & & & & & \\
\hline & & {$[\mathrm{Q}-(\mathrm{Q})]$} & -0.132 & & & & & & & \\
\hline & & {$[\mathrm{T}-(\mathrm{T})]^{\text {core }}$} & -0.034 & & & & & & & \\
\hline & & {$[(\mathrm{Q})-\mathrm{T}]^{\text {core }}$} & 0.009 & & & & & & & \\
\hline \multirow[t]{4}{*}{$\mathrm{C}_{4} \mathrm{H}_{4}$} & tetrahedrane & {$[\mathrm{T}-(\mathrm{T})]$} & -0.692 & -1.014 & -1.490 & -1.516 & -1.719 & & & \\
\hline & & {$[(\mathrm{Q})-\mathrm{T}]$} & 1.026 & & 1.167 & 1.198 & & & & \\
\hline & & {$[\mathrm{T}-(\mathrm{T})]^{\text {core }}$} & -0.035 & & & & & & & \\
\hline & & {$[(\mathrm{Q})-\mathrm{T}]^{\text {core }}$} & 0.006 & & & & & & & \\
\hline
\end{tabular}


Table S10, contd.

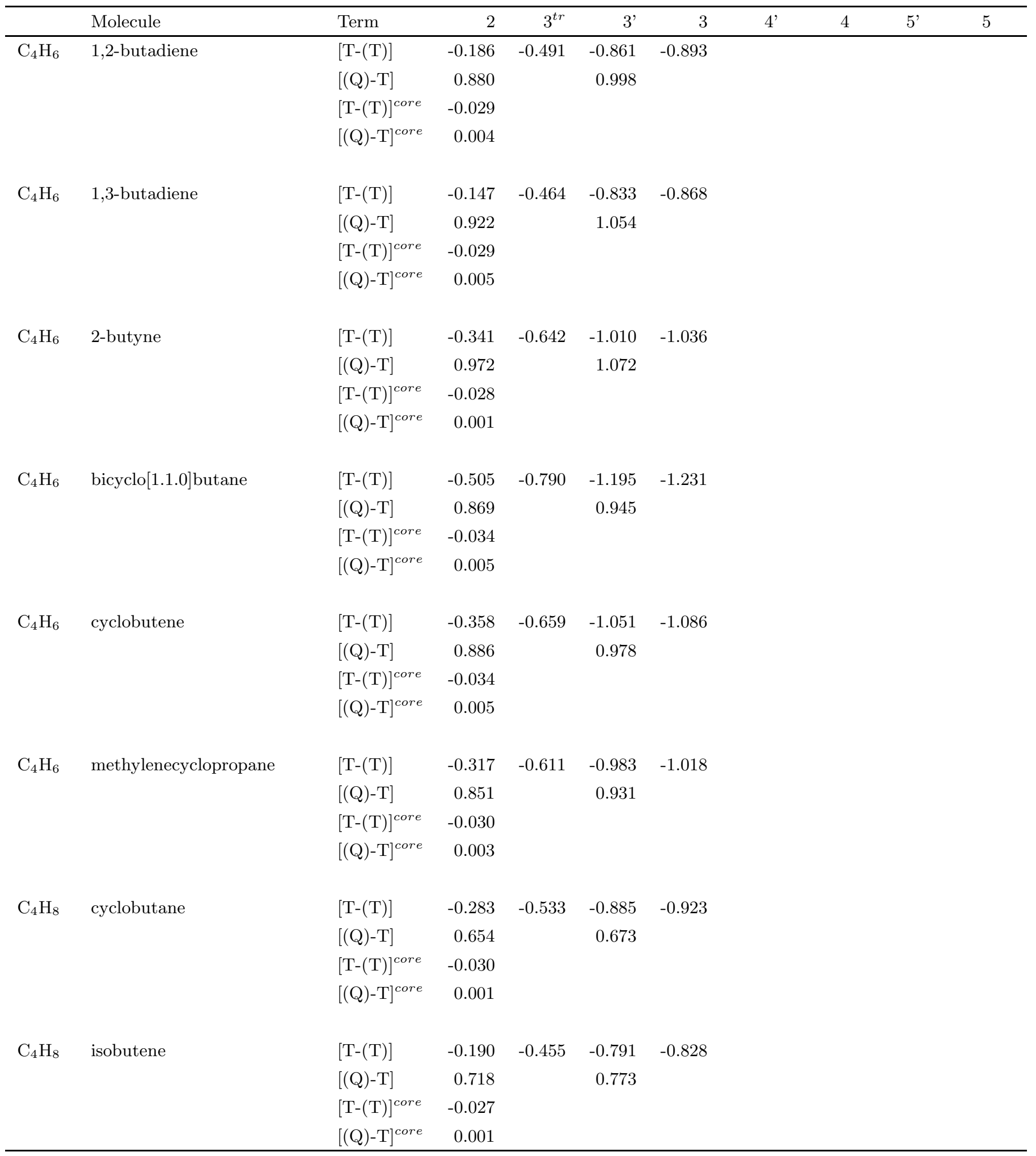


Table S10, contd.

\begin{tabular}{|c|c|c|c|c|c|c|c|c|c|c|}
\hline & Molecule & Term & 2 & $3^{t r}$ & $3^{\prime}$ & 3 & $4^{\prime}$ & 4 & $5^{\prime}$ & 5 \\
\hline $\mathrm{C}_{4} \mathrm{H}_{10}$ & isobutane & {$[\mathrm{T}-(\mathrm{T})]$} & -0.160 & -0.378 & & & & & & \\
\hline \multirow[t]{3}{*}{$\mathrm{C}_{4} \mathrm{H}_{10}$} & $n$-butane & {$[\mathrm{T}-(\mathrm{T})]$} & -0.140 & -0.359 & -0.666 & -0.702 & & & & \\
\hline & & {$[\mathrm{T}-(\mathrm{T})]^{\text {core }}$} & -0.025 & & & & & & & \\
\hline & & {$[(\mathrm{Q})-\mathrm{T}]^{\text {core }}$} & -0.002 & & & & & & & \\
\hline \multirow[t]{2}{*}{$\mathrm{C}_{5} \mathrm{H}_{4}$} & 1,4-pentadiyne & {$[\mathrm{T}-(\mathrm{T})]$} & -0.604 & -1.069 & -1.602 & & & & & \\
\hline & & {$[(\mathrm{Q})-\mathrm{T}]$} & 1.592 & & 1.815 & & & & & \\
\hline \multirow[t]{4}{*}{$\mathrm{C}_{5} \mathrm{H}_{4}$} & pentatetraene & {$[\mathrm{T}-(\mathrm{T})]$} & -0.316 & -0.775 & -1.322 & -1.349 & & & & \\
\hline & & {$[(\mathrm{Q})-\mathrm{T}]$} & 1.766 & & 2.127 & & & & & \\
\hline & & {$[\mathrm{T}-(\mathrm{T})]^{\text {core }}$} & -0.039 & & & & & & & \\
\hline & & {$[(\mathrm{Q})-\mathrm{T}]^{\text {core }}$} & 0.017 & & & & & & & \\
\hline \multirow[t]{3}{*}{$\mathrm{C}_{5} \mathrm{H}_{4}$} & spiropentadiene & {$[\mathrm{T}-(\mathrm{T})]$} & -0.791 & -1.250 & -1.820 & -1.848 & & & & \\
\hline & & {$[(\mathrm{Q})-\mathrm{T}]$} & 1.544 & & 1.777 & & & & & \\
\hline & & {$[\mathrm{T}-(\mathrm{T})]^{\text {core }}$} & -0.049 & & & & & & & \\
\hline $\mathrm{C}_{5} \mathrm{H}_{6}$ & & {$[(\mathrm{Q})-\mathrm{T}]$} & 1.309 & & 1.499 & & & & & \\
\hline \multirow[t]{2}{*}{$\mathrm{C}_{5} \mathrm{H}_{6}$} & 3-methylenecyclobutene & {$[\mathrm{T}-(\mathrm{T})]$} & -0.501 & -0.905 & & & & & & \\
\hline & & {$[(\mathrm{Q})-\mathrm{T}]$} & 1.295 & & & & & & & \\
\hline \multirow[t]{2}{*}{$\mathrm{C}_{5} \mathrm{H}_{6}$} & bicyclo[2.1.0]pent-2-ene & {$[\mathrm{T}-(\mathrm{T})]$} & -0.699 & -1.102 & & & & & & \\
\hline & & {$[(\mathrm{Q})-\mathrm{T}]$} & 1.317 & & & & & & & \\
\hline \multirow[t]{2}{*}{$\mathrm{C}_{5} \mathrm{H}_{6}$} & cyclopropylacetylene & {$[\mathrm{T}-(\mathrm{T})]$} & -0.602 & -1.000 & & & & & & \\
\hline & & {$[(\mathrm{Q})-\mathrm{T}]$} & 1.303 & & & & & & & \\
\hline
\end{tabular}


Table S10, contd.

\begin{tabular}{|c|c|c|c|c|c|c|c|c|c|c|}
\hline & Molecule & Term & 2 & $3^{t r}$ & $3^{\prime}$ & 3 & $4^{\prime}$ & 4 & $5^{\prime}$ & 5 \\
\hline \multirow[t]{2}{*}{$\mathrm{C}_{5} \mathrm{H}_{8}$} & 1,4-pentadiene & {$[\mathrm{T}-(\mathrm{T})]$} & -0.281 & -0.655 & & & & & & \\
\hline & & {$[(\mathrm{Q})-\mathrm{T}]$} & 1.054 & & & & & & & \\
\hline \multirow[t]{2}{*}{$\mathrm{C}_{5} \mathrm{H}_{8}$} & isoprene & {$[\mathrm{T}-(\mathrm{T})]$} & -0.281 & -0.657 & & & & & & \\
\hline & & {$[(\mathrm{Q})-\mathrm{T}]$} & 1.119 & & & & & & & \\
\hline \multirow[t]{2}{*}{$\mathrm{C}_{5} \mathrm{H}_{8}$} & spiropentane & {$[\mathrm{T}-(\mathrm{T})]$} & -0.562 & -0.892 & -1.611 & -1.411 & & & & \\
\hline & & {$[(\mathrm{Q})-\mathrm{T}]$} & 0.962 & & 1.423 & & & & & \\
\hline \multirow[t]{2}{*}{$\mathrm{C}_{5} \mathrm{H}_{10}$} & cyclopentane & {$[\mathrm{T}-(\mathrm{T})]$} & -0.380 & -0.678 & & & & & & \\
\hline & & {$[(\mathrm{Q})-\mathrm{T}]$} & 0.791 & & & & & & & \\
\hline \multirow[t]{2}{*}{$\mathrm{C}_{5} \mathrm{H}_{12}$} & neopentane & {$[\mathrm{T}-(\mathrm{T})]$} & -0.284 & -0.562 & & & & & & \\
\hline & & {$[(\mathrm{Q})-\mathrm{T}]$} & 0.715 & & & & & & & \\
\hline \multirow[t]{2}{*}{$\mathrm{C}_{5} \mathrm{H}_{12}$} & $n$-pentane & {$[\mathrm{T}-(\mathrm{T})]$} & -0.216 & -0.492 & & & & & & \\
\hline & & {$[(\mathrm{Q})-\mathrm{T}]$} & 0.684 & & & & & & & \\
\hline \multirow[t]{4}{*}{$\mathrm{C}_{6} \mathrm{H}_{2}$} & 1,3,5-hexatriyne & {$[\mathrm{T}-(\mathrm{T})]$} & -1.130 & -1.750 & -2.492 & -2.503 & & & & \\
\hline & & {$[(\mathrm{Q})-\mathrm{T}]$} & 2.792 & & 3.268 & & & & & \\
\hline & & {$[\mathrm{T}-(\mathrm{T})]^{\text {core }}$} & -0.057 & & & & & & & \\
\hline & & {$[(\mathrm{Q})-\mathrm{T}]^{\text {core }}$} & 0.018 & & & & & & & \\
\hline \multirow[t]{4}{*}{$\mathrm{C}_{6} \mathrm{H}_{4}$} & butalene & {$[\mathrm{T}-(\mathrm{T})]$} & -1.477 & -2.078 & -2.885 & & & & & \\
\hline & & {$[(\mathrm{Q})-\mathrm{T}]$} & 2.556 & & 3.037 & & & & & \\
\hline & & {$[\mathrm{T}-(\mathrm{T})]^{\text {core }}$} & -0.066 & & & & & & & \\
\hline & & {$[(\mathrm{Q})-\mathrm{T}]^{\text {core }}$} & 0.036 & & & & & & & \\
\hline \multirow[t]{2}{*}{$\mathrm{C}_{6} \mathrm{H}_{4}$} & cis-3-hexene-1,5-diyne & {$[\mathrm{T}-(\mathrm{T})]$} & -0.764 & -1.344 & & & & & & \\
\hline & & {$[(\mathrm{Q})-\mathrm{T}]$} & 2.142 & & & & & & & \\
\hline \multirow[t]{4}{*}{$\mathrm{C}_{6} \mathrm{H}_{4}$} & hexapentaene & {$[\mathrm{T}-(\mathrm{T})]$} & -0.381 & -0.956 & -1.647 & -1.675 & & & & \\
\hline & & {$[(\mathrm{Q})-\mathrm{T}]$} & 2.458 & & 2.984 & & & & & \\
\hline & & {$[\mathrm{T}-(\mathrm{T})]^{\text {core }}$} & -0.045 & & & & & & & \\
\hline & & {$[(\mathrm{Q})-\mathrm{T}]^{\text {core }}$} & 0.028 & & & & & & & \\
\hline \multirow[t]{2}{*}{$\mathrm{C}_{6} \mathrm{H}_{4}$} & ortho-benzyne & {$[\mathrm{T}-(\mathrm{T})]$} & -1.361 & -1.960 & -2.731 & -2.766 & & & & \\
\hline & & {$[(\mathrm{Q})-\mathrm{T}]$} & 2.681 & & 3.169 & & & & & \\
\hline
\end{tabular}


Table S10, contd.

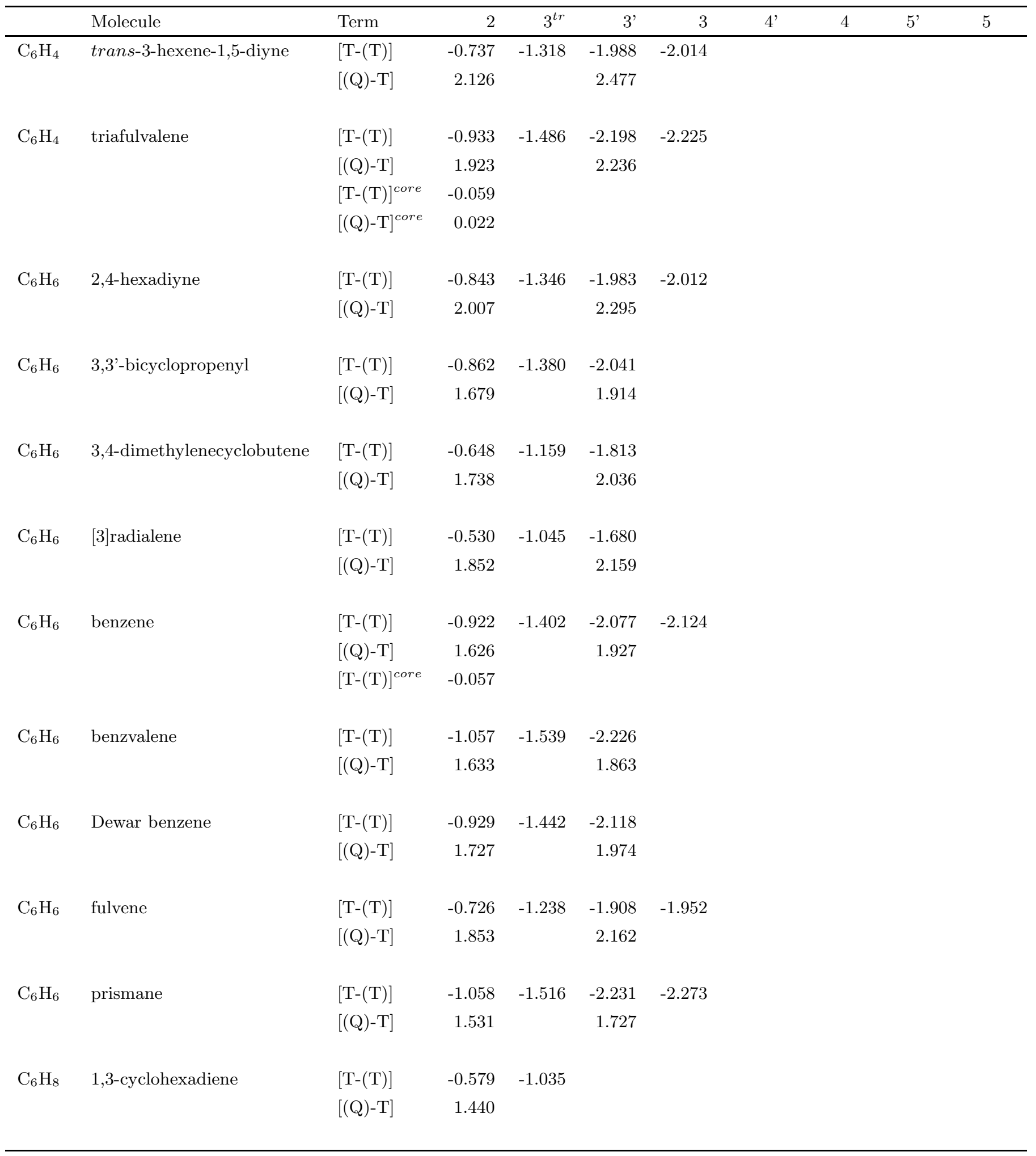


Table S10, contd.

\begin{tabular}{|c|c|c|c|c|c|c|c|c|c|c|}
\hline & Molecule & Term & 2 & $3^{t r}$ & 3 & 3 & $44^{\prime}$ & 4 & 5 & 5 \\
\hline \multirow[t]{2}{*}{$\mathrm{C}_{6} \mathrm{H}_{8}$} & 1,4-cyclohexadiene & {$[\mathrm{T}-(\mathrm{T})]$} & -0.560 & -1.003 & -1.593 & -1.648 & & & & \\
\hline & & {$[(\mathrm{Q})-\mathrm{T}]$} & 1.339 & & 1.505 & & & & & \\
\hline \multirow[t]{2}{*}{$\mathrm{C}_{6} \mathrm{H}_{8}$} & trans-1,3,5-hexatriene & {$[\mathrm{T}-(\mathrm{T})]$} & -0.312 & -0.797 & & & & & & \\
\hline & & {$[(\mathrm{Q})-\mathrm{T}]$} & 1.542 & & & & & & & \\
\hline \multirow[t]{2}{*}{$\mathrm{C}_{6} \mathrm{H}_{10}$} & cyclohexene & {$[\mathrm{T}-(\mathrm{T})]$} & -0.514 & -0.912 & & & & & & \\
\hline & & {$[(\mathrm{Q})-\mathrm{T}]$} & 1.140 & & & & & & & \\
\hline \multirow[t]{2}{*}{$\mathrm{C}_{6} \mathrm{H}_{12}$} & cyclohexane & {$[\mathrm{T}-(\mathrm{T})]$} & -0.459 & -0.806 & & & & & & \\
\hline & & {$[(\mathrm{Q})-\mathrm{T}]$} & 0.935 & & & & & & & \\
\hline \multirow[t]{2}{*}{$\mathrm{C}_{7} \mathrm{H}_{4}$} & 3,3-diethynylcyclopropene & {$[\mathrm{T}-(\mathrm{T})]$} & -1.214 & -1.889 & & & & & & \\
\hline & & {$[(\mathrm{Q})-\mathrm{T}]$} & 2.469 & & & & & & & \\
\hline \multirow[t]{2}{*}{$\mathrm{C}_{7} \mathrm{H}_{4}$} & heptahexaene & {$[\mathrm{T}-(\mathrm{T})]$} & -0.592 & -1.277 & & & & & & \\
\hline & & {$[(\mathrm{Q})-\mathrm{T}]$} & 3.171 & & & & & & & \\
\hline \multirow[t]{2}{*}{$\mathrm{C}_{7} \mathrm{H}_{6}$} & 1,1-diethynylcyclopropane & {$[\mathrm{T}-(\mathrm{T})]$} & -1.081 & -1.694 & & & & & & \\
\hline & & {$[(\mathrm{Q})-\mathrm{T}]$} & 2.179 & & & & & & & \\
\hline \multirow[t]{2}{*}{$\mathrm{C}_{7} \mathrm{H}_{6}$} & 1,3,5-norcaratriene & {$[\mathrm{T}-(\mathrm{T})]$} & -1.372 & -1.963 & & & & & & \\
\hline & & {$[(\mathrm{Q})-\mathrm{T}]$} & 2.139 & & & & & & & \\
\hline \multirow[t]{2}{*}{$\mathrm{C}_{7} \mathrm{H}_{6}$} & fulvenallene & {$[\mathrm{T}-(\mathrm{T})]$} & -0.930 & -1.543 & & & & & & \\
\hline & & {$[(\mathrm{Q})-\mathrm{T}]$} & 2.247 & & & & & & & \\
\hline \multirow[t]{2}{*}{$\mathrm{C}_{7} \mathrm{H}_{6}$} & spiro[2.4]hepta-1,4,6-triene & {$[\mathrm{T}-(\mathrm{T})]$} & -1.181 & -1.795 & & & & & & \\
\hline & & {$[(\mathrm{Q})-\mathrm{T}]$} & 2.197 & & & & & & & \\
\hline \multirow[t]{2}{*}{$\mathrm{C}_{7} \mathrm{H}_{8}$} & 1,3,5-cycloheptatriene & {$[\mathrm{T}-(\mathrm{T})]$} & -0.926 & -1.504 & & & & & & \\
\hline & & {$[(\mathrm{Q})-\mathrm{T}]$} & 2.028 & & & & & & & \\
\hline \multirow[t]{2}{*}{$\mathrm{C}_{7} \mathrm{H}_{8}$} & 2,5-norbornadiene & {$[\mathrm{T}-(\mathrm{T})]$} & -1.100 & -1.661 & & & & & & \\
\hline & & {$[(\mathrm{Q})-\mathrm{T}]$} & 1.900 & & & & & & & \\
\hline \multirow[t]{2}{*}{$\mathrm{C}_{7} \mathrm{H}_{8}$} & norcaradiene & {$[\mathrm{T}-(\mathrm{T})]$} & -1.008 & -1.558 & & & & & & \\
\hline & & {$[(\mathrm{Q})-\mathrm{T}]$} & 1.908 & & & & & & & \\
\hline
\end{tabular}


Table S10, contd.

\begin{tabular}{|c|c|c|c|c|c|c|c|c|c|c|}
\hline & Molecule & Term & 2 & $3^{t r}$ & 3 & 3 & $4 '$ & 4 & 5 & 5 \\
\hline \multirow[t]{2}{*}{$\mathrm{C}_{7} \mathrm{H}_{8}$} & quadricyclane & {$[\mathrm{T}-(\mathrm{T})]$} & -1.235 & -1.730 & & & & & & \\
\hline & & {$[(\mathrm{Q})-\mathrm{T}]$} & 1.633 & & & & & & & \\
\hline \multirow[t]{2}{*}{$\mathrm{C}_{7} \mathrm{H}_{8}$} & spiro[2.4] hepta-4,6-diene & {$[\mathrm{T}-(\mathrm{T})]$} & -1.047 & -1.602 & & & & & & \\
\hline & & {$[(\mathrm{Q})-\mathrm{T}]$} & 1.899 & & & & & & & \\
\hline \multirow[t]{2}{*}{$\mathrm{C}_{8} \mathrm{H}_{2}$} & 1,3,5,7-octatetrayne & {$[\mathrm{T}-(\mathrm{T})]$} & -1.731 & -2.564 & & & & & & \\
\hline & & {$[(\mathrm{Q})-\mathrm{T}]$} & 4.057 & & & & & & & \\
\hline \multirow[t]{2}{*}{$\mathrm{C}_{8} \mathrm{H}_{4}$} & 1,2,3,5,6,7-cyclooctahexaene & {$[\mathrm{T}-(\mathrm{T})]$} & -0.776 & -1.639 & & & & & & \\
\hline & & {$[(\mathrm{Q})-\mathrm{T}]$} & 3.773 & & & & & & & \\
\hline \multirow[t]{2}{*}{$\mathrm{C}_{8} \mathrm{H}_{4}$} & 1,5-cyclooctadiene-3,7-diyne & {$[\mathrm{T}-(\mathrm{T})]$} & -1.438 & -2.252 & & & & & & \\
\hline & & {$[(\mathrm{Q})-\mathrm{T}]$} & 3.592 & & & & & & & \\
\hline \multirow[t]{2}{*}{$\mathrm{C}_{8} \mathrm{H}_{4}$} & octaheptaene & {$[\mathrm{T}-(\mathrm{T})]$} & -0.645 & -1.451 & & & & & & \\
\hline & & {$[(\mathrm{Q})-\mathrm{T}]$} & 3.984 & & & & & & & \\
\hline \multirow[t]{2}{*}{$\mathrm{C}_{8} \mathrm{H}_{6}$} & benzocyclobutadiene & {$[\mathrm{T}-(\mathrm{T})]$} & -1.388 & -2.092 & & & & & & \\
\hline & & {$[(\mathrm{Q})-\mathrm{T}]$} & 2.653 & & & & & & & \\
\hline \multirow[t]{2}{*}{$\mathrm{C}_{8} \mathrm{H}_{6}$} & bis(cyclopropa)benzene & {$[\mathrm{T}-(\mathrm{T})]$} & -1.860 & -2.564 & & & & & & \\
\hline & & {$[(\mathrm{Q})-\mathrm{T}]$} & 2.669 & & & & & & & \\
\hline \multirow[t]{2}{*}{$\mathrm{C}_{8} \mathrm{H}_{6}$} & pentalene & {$[\mathrm{T}-(\mathrm{T})]$} & -1.308 & -2.027 & & & & & & \\
\hline & & {$[(\mathrm{Q})-\mathrm{T}]$} & 2.959 & & & & & & & \\
\hline \multirow[t]{2}{*}{$\mathrm{C}_{8} \mathrm{H}_{8}$} & 1,3,5,7-cyclooctatetraene & {$[\mathrm{T}-(\mathrm{T})]$} & -0.946 & -1.627 & & & & & & \\
\hline & & {$[(\mathrm{Q})-\mathrm{T}]$} & 2.299 & & & & & & & \\
\hline \multirow[t]{2}{*}{$\mathrm{C}_{8} \mathrm{H}_{8}$} & benzocyclobutene & {$[\mathrm{T}-(\mathrm{T})]$} & -1.420 & -2.052 & & & & & & \\
\hline & & {$[(\mathrm{Q})-\mathrm{T}]$} & 2.199 & & & & & & & \\
\hline \multirow[t]{2}{*}{$\mathrm{C}_{8} \mathrm{H}_{8}$} & cubane & {$[\mathrm{T}-(\mathrm{T})]$} & -1.382 & -1.972 & & & & & & \\
\hline & & {$[(\mathrm{Q})-\mathrm{T}]$} & 2.002 & & & & & & & \\
\hline \multirow[t]{2}{*}{$\mathrm{C}_{8} \mathrm{H}_{8}$} & para-xylylene & {$[\mathrm{T}-(\mathrm{T})]$} & -0.735 & -1.410 & & & & & & \\
\hline & & {$[(\mathrm{Q})-\mathrm{T}]$} & 2.557 & & & & & & & \\
\hline
\end{tabular}


Table S10, contd.

\begin{tabular}{|c|c|c|c|c|c|c|c|c|c|c|}
\hline & Molecule & Term & 2 & $3^{t r}$ & 3 & 3 & 41 & 4 & 5 & 5 \\
\hline \multirow[t]{2}{*}{$\mathrm{C}_{10} \mathrm{H}_{4}$} & tetraethynylethene & {$[\mathrm{T}-(\mathrm{T})]$} & -1.794 & -2.819 & & & & & & \\
\hline & & {$[(\mathrm{Q})-\mathrm{T}]$} & 4.178 & & & & & & & \\
\hline \multirow[t]{2}{*}{$\mathrm{C}_{10} \mathrm{H}_{8}$} & naphthalene & {$[\mathrm{T}-(\mathrm{T})]$} & -1.996 & -2.820 & & & & & & \\
\hline & & {$[(\mathrm{Q})-\mathrm{T}]$} & 3.154 & & & & & & & \\
\hline \multirow[t]{2}{*}{$\mathrm{C}_{10} \mathrm{H}_{8}$} & pentafulvalene & {$[\mathrm{T}-(\mathrm{T})]$} & -1.623 & -2.504 & & & & & & \\
\hline & & {$[(\mathrm{Q})-\mathrm{T}]$} & 3.546 & & & & & & & \\
\hline
\end{tabular}

${ }^{a}$ Basis sets are defined by their cardinal number as given in the header row (notation as in Sec 3). See text for further details and for definitions of terms. 
Table S11: Higher order electron correlation contributions to atomization energies (kcal/mol, CBS-extrapolated $)^{a}$

\begin{tabular}{|c|c|c|c|c|c|c|c|c|c|}
\hline & Molecule & Term & $\left(2,3^{t r}\right)$ & $\overline{\mathbf{3}^{\prime}}$ & $\overline{\mathbf{3}}$ & $\overline{\overline{4^{\prime}}}$ & $\overline{4}$ & $\overline{5^{\prime}}$ & $\overline{5}$ \\
\hline \multirow[t]{6}{*}{$\mathrm{CH}_{4}$} & methane & {$[\mathrm{T}-(\mathrm{T})]$} & -0.043 & -0.078 & -0.086 & -0.072 & -0.102 & -0.082 & -0.103 \\
\hline & & {$[(\mathrm{Q})-\mathrm{T}]$} & 0.098 & 0.044 & 0.070 & 0.087 & 0.096 & 0.091 & 0.093 \\
\hline & & {$[\mathrm{Q}-(\mathrm{Q})]$} & & 0.002 & 0.002 & & & & \\
\hline & & {$[(\mathrm{P})-\mathrm{Q}]$} & & -0.006 & -0.007 & & & & \\
\hline & & {$[\mathrm{T}-(\mathrm{T})]^{\text {core }}$} & & & 0.007 & & 0.020 & & \\
\hline & & {$[(\mathrm{Q})-\mathrm{T}]^{\text {core }}$} & & & -0.003 & & -0.006 & & \\
\hline \multirow[t]{6}{*}{$\mathrm{C}_{2} \mathrm{H}_{2}$} & acetylene & {$[\mathrm{T}-(\mathrm{T})]$} & -0.553 & -0.715 & -0.728 & -0.715 & -0.734 & -0.733 & -0.746 \\
\hline & & {$[(\mathrm{Q})-\mathrm{T}]$} & 0.788 & 0.750 & 0.769 & 0.820 & 0.827 & 0.816 & 0.817 \\
\hline & & {$[\mathrm{Q}-(\mathrm{Q})]$} & & -0.060 & -0.062 & & & & \\
\hline & & {$[(\mathrm{P})-\mathrm{Q}]$} & & 0.033 & 0.034 & & & & \\
\hline & & {$[\mathrm{T}-(\mathrm{T})]^{\text {core }}$} & & & 0.018 & & 0.043 & & \\
\hline & & {$[(\mathrm{Q})-\mathrm{T}]^{\text {core }}$} & & & 0.018 & & 0.012 & & \\
\hline \multirow[t]{6}{*}{$\mathrm{C}_{2} \mathrm{H}_{4}$} & ethylene & {$[\mathrm{T}-(\mathrm{T})]$} & -0.298 & -0.436 & -0.461 & -0.444 & -0.480 & -0.461 & \\
\hline & & {$[(\mathrm{Q})-\mathrm{T}]$} & 0.455 & 0.409 & 0.445 & 0.484 & 0.497 & 0.488 & \\
\hline & & {$[\mathrm{Q}-(\mathrm{Q})]$} & & -0.019 & -0.020 & & & & \\
\hline & & {$[(\mathrm{P})-\mathrm{Q}]$} & & -0.003 & & & & & \\
\hline & & {$[\mathrm{T}-(\mathrm{T})]^{\text {core }}$} & & & 0.018 & & & & \\
\hline & & {$[(\mathrm{Q})-\mathrm{T}]^{\text {core }}$} & & & 0.009 & & & & \\
\hline \multirow[t]{6}{*}{$\mathrm{C}_{2} \mathrm{H}_{6}$} & ethane & {$[\mathrm{T}-(\mathrm{T})]$} & -0.204 & -0.328 & -0.350 & -0.330 & -0.379 & & \\
\hline & & {$[(\mathrm{Q})-\mathrm{T}]$} & 0.272 & 0.182 & 0.227 & 0.265 & 0.281 & & \\
\hline & & {$[\mathrm{Q}-(\mathrm{Q})]$} & & -0.004 & & & & & \\
\hline & & {$[(\mathrm{P})-\mathrm{Q}]$} & & -0.014 & & & & & \\
\hline & & {$[\mathrm{T}-(\mathrm{T})]^{\text {core }}$} & & & 0.008 & & & & \\
\hline & & {$[(\mathrm{Q})-\mathrm{T}]^{\text {core }}$} & & & 0.000 & & & & \\
\hline \multirow[t]{4}{*}{$\mathrm{C}_{3} \mathrm{H}_{4}$} & allene & {$[\mathrm{T}-(\mathrm{T})]$} & -0.560 & -0.815 & -0.848 & -0.837 & -0.877 & & \\
\hline & & {$[(\mathrm{Q})-\mathrm{T}]$} & 0.858 & 0.834 & 0.877 & 0.944 & & & \\
\hline & & {$[\mathrm{T}-(\mathrm{T})]^{\text {core }}$} & & & 0.027 & & & & \\
\hline & & {$[(\mathrm{Q})-\mathrm{T}]^{\text {core }}$} & & & 0.024 & & & & \\
\hline \multirow[t]{4}{*}{$\mathrm{C}_{3} \mathrm{H}_{4}$} & cyclopropene & {$[\mathrm{T}-(\mathrm{T})]$} & -0.789 & -1.068 & -1.101 & -1.087 & -1.126 & & \\
\hline & & {$[(\mathrm{Q})-\mathrm{T}]$} & 0.940 & 0.874 & 0.916 & 0.990 & & & \\
\hline & & {$[\mathrm{T}-(\mathrm{T})]^{\text {core }}$} & & & 0.012 & & & & \\
\hline & & {$[(\mathrm{Q})-\mathrm{T}]^{\text {core }}$} & & & 0.025 & & & & \\
\hline
\end{tabular}


Table S11, contd.

\begin{tabular}{|c|c|c|c|c|c|c|c|c|c|}
\hline & Molecule & Term & $\left(2,3^{t r}\right)$ & $\overline{3^{\prime}}$ & $\overline{3}$ & $\overline{\overline{4^{\prime}}}$ & $\overline{4}$ & $\overline{5^{\prime}}$ & $\overline{5}$ \\
\hline \multirow[t]{3}{*}{$\mathrm{C}_{3} \mathrm{H}_{4}$} & propyne & {$[\mathrm{T}-(\mathrm{T})]$} & -0.744 & -0.997 & -1.023 & -1.006 & & & \\
\hline & & {$[(\mathrm{Q})-\mathrm{T}]$} & 0.997 & 0.927 & 0.965 & 1.037 & & & \\
\hline & & {$[\mathrm{T}-(\mathrm{T})]^{\text {core }}$} & & & 0.022 & & & & \\
\hline \multirow[t]{3}{*}{$\mathrm{C}_{3} \mathrm{H}_{6}$} & cyclopropane & {$[\mathrm{T}-(\mathrm{T})]$} & -0.580 & -0.837 & -0.876 & -0.858 & & & \\
\hline & & {$[(\mathrm{Q})-\mathrm{T}]$} & 0.622 & 0.511 & 0.567 & 0.637 & & & \\
\hline & & {$[\mathrm{T}-(\mathrm{T})]^{\text {core }}$} & & & 0.005 & & & & \\
\hline \multirow[t]{2}{*}{$\mathrm{C}_{3} \mathrm{H}_{6}$} & propene & {$[\mathrm{T}-(\mathrm{T})]$} & -0.496 & -0.723 & -0.761 & & & & \\
\hline & & {$[(\mathrm{Q})-\mathrm{T}]$} & 0.672 & 0.594 & & & & & \\
\hline \multirow[t]{2}{*}{$\mathrm{C}_{3} \mathrm{H}_{8}$} & propane & {$[\mathrm{T}-(\mathrm{T})]$} & -0.387 & -0.603 & -0.640 & -0.616 & & & \\
\hline & & {$[(\mathrm{Q})-\mathrm{T}]$} & 0.464 & 0.344 & & & & & \\
\hline \multirow[t]{4}{*}{$\mathrm{C}_{4} \mathrm{H}_{2}$} & 1,3-butadiyne & {$[\mathrm{T}-(\mathrm{T})]$} & -1.407 & -1.828 & -1.844 & -1.834 & -1.854 & & \\
\hline & & {$[(\mathrm{Q})-\mathrm{T}]$} & 2.040 & 2.010 & 2.030 & 2.139 & 2.146 & & \\
\hline & & {$[\mathrm{T}-(\mathrm{T})]^{\text {core }}$} & & & 0.039 & & & & \\
\hline & & {$[(\mathrm{Q})-\mathrm{T}]^{\text {core }}$} & & & 0.049 & & & & \\
\hline \multirow[t]{2}{*}{$\mathrm{C}_{4} \mathrm{H}_{4}$} & bicyclo[1.1.0]but-1(3)-ene & {$[\mathrm{T}-(\mathrm{T})]$} & -1.426 & -1.856 & -1.898 & & & & \\
\hline & & {$[(\mathrm{Q})-\mathrm{T}]$} & 2.204 & 2.078 & & & & & \\
\hline \multirow[t]{2}{*}{$\mathrm{C}_{4} \mathrm{H}_{4}$} & butatriene & {$[\mathrm{T}-(\mathrm{T})]$} & -0.854 & -1.229 & -1.266 & -1.265 & & & \\
\hline & & {$[(\mathrm{Q})-\mathrm{T}]$} & 1.512 & 1.545 & 1.592 & 1.687 & & & \\
\hline \multirow[t]{2}{*}{$\mathrm{C}_{4} \mathrm{H}_{4}$} & cyclobutadiene & {$[\mathrm{T}-(\mathrm{T})]$} & -0.949 & -1.353 & -1.394 & -1.381 & -1.424 & & \\
\hline & & {$[(\mathrm{Q})-\mathrm{T}]$} & 1.664 & 1.647 & 1.699 & 1.789 & & & \\
\hline \multirow[t]{2}{*}{$\mathrm{C}_{4} \mathrm{H}_{4}$} & ethynylethene & {$[\mathrm{T}-(\mathrm{T})]$} & -1.077 & -1.448 & -1.482 & & & & \\
\hline & & {$[(\mathrm{Q})-\mathrm{T}]$} & 1.513 & 1.472 & & & & & \\
\hline \multirow[t]{2}{*}{$\mathrm{C}_{4} \mathrm{H}_{4}$} & methylenecyclopropene & {$[\mathrm{T}-(\mathrm{T})]$} & -1.093 & -1.497 & -1.534 & & & & \\
\hline & & {$[(\mathrm{Q})-\mathrm{T}]$} & 1.326 & 1.282 & & & & & \\
\hline \multirow[t]{2}{*}{$\mathrm{C}_{4} \mathrm{H}_{4}$} & tetrahedrane & {$[\mathrm{T}-(\mathrm{T})]$} & -1.336 & -1.826 & -1.863 & -1.867 & & & \\
\hline & & {$[(\mathrm{Q})-\mathrm{T}]$} & 1.282 & 1.226 & 1.271 & & & & \\
\hline \multirow[t]{2}{*}{$\mathrm{C}_{4} \mathrm{H}_{6}$} & 1,2-butadiene & {$[\mathrm{T}-(\mathrm{T})]$} & -0.797 & -1.145 & -1.190 & & & & \\
\hline & & {$[(\mathrm{Q})-\mathrm{T}]$} & 1.100 & 1.047 & & & & & \\
\hline
\end{tabular}


Table S11, contd.

\begin{tabular}{|c|c|c|c|c|c|c|c|c|c|}
\hline & Molecule & Term & $\left(2,3^{\text {tr }}\right)$ & $\overline{\mathbf{3}^{\prime}}$ & $\overline{3}$ & $\overline{\overline{4^{\prime}}}$ & $\overline{4}$ & $\overline{\overline{5^{\prime}}}$ & $\overline{5}$ \\
\hline \multirow[t]{2}{*}{$\mathrm{C}_{4} \mathrm{H}_{6}$} & 1,3-butadiene & {$[\mathrm{T}-(\mathrm{T})]$} & -0.781 & -1.123 & -1.172 & & & & \\
\hline & & {$[(\mathrm{Q})-\mathrm{T}]$} & 1.153 & 1.109 & & & & & \\
\hline \multirow[t]{2}{*}{$\mathrm{C}_{4} \mathrm{H}_{6}$} & 2-butyne & {$[\mathrm{T}-(\mathrm{T})]$} & -0.944 & -1.292 & -1.329 & & & & \\
\hline & & {$[(\mathrm{Q})-\mathrm{T}]$} & 1.215 & 1.114 & & & & & \\
\hline \multirow[t]{2}{*}{$\mathrm{C}_{4} \mathrm{H}_{6}$} & bicyclo[1.1.0]butane & {$[\mathrm{T}-(\mathrm{T})]$} & -1.075 & -1.486 & -1.537 & & & & \\
\hline & & {$[(\mathrm{Q})-\mathrm{T}]$} & 1.087 & 0.977 & & & & & \\
\hline \multirow[t]{2}{*}{$\mathrm{C}_{4} \mathrm{H}_{6}$} & cyclobutene & {$[\mathrm{T}-(\mathrm{T})]$} & -0.961 & -1.343 & -1.393 & & & & \\
\hline & & {$[(\mathrm{Q})-\mathrm{T}]$} & 1.108 & 1.016 & & & & & \\
\hline \multirow[t]{2}{*}{$\mathrm{C}_{4} \mathrm{H}_{6}$} & methylenecyclopropane & {$[\mathrm{T}-(\mathrm{T})]$} & -0.904 & -1.264 & -1.313 & & & & \\
\hline & & {$[(\mathrm{Q})-\mathrm{T}]$} & 1.063 & 0.965 & & & & & \\
\hline \multirow[t]{2}{*}{$\mathrm{C}_{4} \mathrm{H}_{8}$} & cyclobutane & {$[\mathrm{T}-(\mathrm{T})]$} & -0.782 & -1.139 & -1.192 & & & & \\
\hline & & {$[(\mathrm{Q})-\mathrm{T}]$} & 0.817 & 0.681 & & & & & \\
\hline \multirow[t]{2}{*}{$\mathrm{C}_{4} \mathrm{H}_{8}$} & isobutene & {$[\mathrm{T}-(\mathrm{T})]$} & -0.721 & -1.045 & -1.096 & & & & \\
\hline & & {$[(\mathrm{Q})-\mathrm{T}]$} & 0.898 & 0.797 & & & & & \\
\hline \multirow[t]{2}{*}{$\mathrm{C}_{4} \mathrm{H}_{10}$} & isobutane & {$[\mathrm{T}-(\mathrm{T})]$} & -0.597 & & & & & & \\
\hline & & {$[(\mathrm{Q})-\mathrm{T}]$} & 0.671 & & & & & & \\
\hline \multirow[t]{2}{*}{$\mathrm{C}_{4} \mathrm{H}_{10}$} & $n$-butane & {$[\mathrm{T}-(\mathrm{T})]$} & -0.577 & -0.888 & -0.938 & & & & \\
\hline & & {$[(\mathrm{Q})-\mathrm{T}]$} & 0.658 & 0.511 & & & & & \\
\hline \multirow[t]{2}{*}{$\mathrm{C}_{5} \mathrm{H}_{4}$} & 1,4-pentadiyne & {$[\mathrm{T}-(\mathrm{T})]$} & -1.534 & -2.023 & & & & & \\
\hline & & {$[(\mathrm{Q})-\mathrm{T}]$} & 1.991 & 1.908 & & & & & \\
\hline \multirow[t]{2}{*}{$\mathrm{C}_{5} \mathrm{H}_{4}$} & pentatetraene & {$[\mathrm{T}-(\mathrm{T})]$} & -1.233 & -1.746 & -1.784 & & & & \\
\hline & & {$[(\mathrm{Q})-\mathrm{T}]$} & 2.208 & 2.279 & & & & & \\
\hline \multirow[t]{2}{*}{$\mathrm{C}_{5} \mathrm{H}_{4}$} & spiropentadiene & {$[\mathrm{T}-(\mathrm{T})]$} & -1.710 & -2.254 & -2.293 & & & & \\
\hline & & {$[(\mathrm{Q})-\mathrm{T}]$} & 1.931 & 1.876 & & & & & \\
\hline \multirow[t]{2}{*}{$\mathrm{C}_{5} \mathrm{H}_{6}$} & [1.1.1]propellane & {$[\mathrm{T}-(\mathrm{T})]$} & -1.840 & -2.416 & -2.472 & & & & \\
\hline & & {$[(\mathrm{Q})-\mathrm{T}]$} & 1.954 & 1.844 & & & & & \\
\hline
\end{tabular}


Table S11, contd.

\begin{tabular}{|c|c|c|c|c|c|c|c|c|c|}
\hline & Molecule & Term & $\left(2,3^{t r}\right)$ & $\overline{\overline{3^{\prime}}}$ & $\overline{3}$ & $\overline{\overline{4^{\prime}}}$ & $\overline{4}$ & $\overline{\overline{5^{\prime}}}$ & $\overline{\overline{5}}$ \\
\hline \multirow[t]{2}{*}{$\mathrm{C}_{5} \mathrm{H}_{6}$} & 1,3-cyclopentadiene & {$[\mathrm{T}-(\mathrm{T})]$} & -1.360 & -1.868 & -1.930 & & & & \\
\hline & & {$[(\mathrm{Q})-\mathrm{T}]$} & 1.636 & 1.580 & & & & & \\
\hline \multirow[t]{2}{*}{$\mathrm{C}_{5} \mathrm{H}_{6}$} & 3-methylenecyclobutene & {$[\mathrm{T}-(\mathrm{T})]$} & -1.310 & & & & & & \\
\hline & & {$[(\mathrm{Q})-\mathrm{T}]$} & 1.618 & & & & & & \\
\hline \multirow[t]{2}{*}{$\mathrm{C}_{5} \mathrm{H}_{6}$} & bicyclo[2.1.0]pent-2-ene & {$[\mathrm{T}-(\mathrm{T})]$} & -1.504 & & & & & & \\
\hline & & {$[(\mathrm{Q})-\mathrm{T}]$} & 1.646 & & & & & & \\
\hline \multirow[t]{2}{*}{$\mathrm{C}_{5} \mathrm{H}_{6}$} & cyclopropylacetylene & {$[\mathrm{T}-(\mathrm{T})]$} & -1.397 & & & & & & \\
\hline & & {$[(\mathrm{Q})-\mathrm{T}]$} & 1.628 & & & & & & \\
\hline \multirow[t]{2}{*}{$\mathrm{C}_{5} \mathrm{H}_{8}$} & 1,4-pentadiene & {$[\mathrm{T}-(\mathrm{T})]$} & -1.029 & & & & & & \\
\hline & & {$[(\mathrm{Q})-\mathrm{T}]$} & 1.317 & & & & & & \\
\hline \multirow[t]{2}{*}{$\mathrm{C}_{5} \mathrm{H}_{8}$} & isoprene & {$[\mathrm{T}-(\mathrm{T})]$} & -1.032 & & & & & & \\
\hline & & {$[(\mathrm{Q})-\mathrm{T}]$} & 1.398 & & & & & & \\
\hline \multirow[t]{2}{*}{$\mathrm{C}_{5} \mathrm{H}_{8}$} & spiropentane & {$[\mathrm{T}-(\mathrm{T})]$} & -1.221 & -2.052 & -1.768 & & & & \\
\hline & & {$[(\mathrm{Q})-\mathrm{T}]$} & 1.203 & 1.617 & & & & & \\
\hline \multirow[t]{2}{*}{$\mathrm{C}_{5} \mathrm{H}_{10}$} & cyclopentane & {$[\mathrm{T}-(\mathrm{T})]$} & -0.976 & & & & & & \\
\hline & & {$[(\mathrm{Q})-\mathrm{T}]$} & 0.988 & & & & & & \\
\hline \multirow[t]{2}{*}{$\mathrm{C}_{5} \mathrm{H}_{12}$} & neopentane & {$[\mathrm{T}-(\mathrm{T})]$} & -0.840 & & & & & & \\
\hline & & {$[(\mathrm{Q})-\mathrm{T}]$} & 0.893 & & & & & & \\
\hline \multirow[t]{2}{*}{$\mathrm{C}_{5} \mathrm{H}_{12}$} & $n$-pentane & {$[\mathrm{T}-(\mathrm{T})]$} & -0.769 & & & & & & \\
\hline & & {$[(\mathrm{Q})-\mathrm{T}]$} & 0.855 & & & & & & \\
\hline \multirow[t]{2}{*}{$\mathrm{C}_{6} \mathrm{H}_{2}$} & 1,3,5-hexatriyne & {$[\mathrm{T}-(\mathrm{T})]$} & -2.371 & -3.066 & -3.082 & & & & \\
\hline & & {$[(\mathrm{Q})-\mathrm{T}]$} & 3.490 & 3.469 & & & & & \\
\hline \multirow[t]{2}{*}{$\mathrm{C}_{6} \mathrm{H}_{4}$} & butalene & {$[\mathrm{T}-(\mathrm{T})]$} & -2.679 & -3.478 & & & & & \\
\hline & & {$[(\mathrm{Q})-\mathrm{T}]$} & 3.195 & 3.239 & & & & & \\
\hline \multirow[t]{2}{*}{$\mathrm{C}_{6} \mathrm{H}_{4}$} & cis-3-hexene-1,5-diyne & {$[\mathrm{T}-(\mathrm{T})]$} & -1.925 & & & & & & \\
\hline & & {$[(\mathrm{Q})-\mathrm{T}]$} & 2.678 & & & & & & \\
\hline
\end{tabular}


Table S11, contd.

\begin{tabular}{|c|c|c|c|c|c|c|c|c|c|}
\hline & Molecule & Term & $\left(2,3^{t r}\right)$ & $\overline{\mathbf{3}^{\prime}}$ & $\overline{3}$ & $\overline{\overline{4^{\prime}}}$ & $\overline{4}$ & $\overline{\overline{5^{\prime}}}$ & $\overline{5}$ \\
\hline \multirow[t]{2}{*}{$\mathrm{C}_{6} \mathrm{H}_{4}$} & hexapentaene & {$[\mathrm{T}-(\mathrm{T})]$} & -1.531 & -2.179 & -2.220 & & & & \\
\hline & & {$[(\mathrm{Q})-\mathrm{T}]$} & 3.072 & 3.205 & & & & & \\
\hline \multirow[t]{2}{*}{$\mathrm{C}_{6} \mathrm{H}_{4}$} & ortho-benzyne & {$[\mathrm{T}-(\mathrm{T})]$} & -2.559 & -3.307 & -3.357 & & & & \\
\hline & & {$[(\mathrm{Q})-\mathrm{T}]$} & 3.352 & 3.374 & & & & & \\
\hline \multirow[t]{2}{*}{$\mathrm{C}_{6} \mathrm{H}_{4}$} & trans-3-hexene-1,5-diyne & {$[\mathrm{T}-(\mathrm{T})]$} & -1.898 & -2.514 & -2.552 & & & & \\
\hline & & {$[(\mathrm{Q})-\mathrm{T}]$} & 2.658 & 2.625 & & & & & \\
\hline \multirow[t]{2}{*}{$\mathrm{C}_{6} \mathrm{H}_{4}$} & triafulvalene & {$[\mathrm{T}-(\mathrm{T})]$} & -2.038 & -2.731 & -2.768 & & & & \\
\hline & & {$[(\mathrm{Q})-\mathrm{T}]$} & 2.403 & 2.368 & & & & & \\
\hline \multirow[t]{2}{*}{$\mathrm{C}_{6} \mathrm{H}_{6}$} & 2,4-hexadiyne & {$[\mathrm{T}-(\mathrm{T})]$} & -1.850 & -2.463 & -2.505 & & & & \\
\hline & & {$[(\mathrm{Q})-\mathrm{T}]$} & 2.509 & 2.417 & & & & & \\
\hline \multirow[t]{2}{*}{$\mathrm{C}_{6} \mathrm{H}_{6}$} & 3,3'-bicyclopropenyl & {$[\mathrm{T}-(\mathrm{T})]$} & -1.898 & -2.537 & & & & & \\
\hline & & {$[(\mathrm{Q})-\mathrm{T}]$} & 2.099 & 2.013 & & & & & \\
\hline \multirow[t]{2}{*}{$\mathrm{C}_{6} \mathrm{H}_{6}$} & 3,4-dimethylenecyclobutene & {$[\mathrm{T}-(\mathrm{T})]$} & -1.670 & -2.304 & & & & & \\
\hline & & {$[(\mathrm{Q})-\mathrm{T}]$} & 2.173 & 2.162 & & & & & \\
\hline \multirow[t]{2}{*}{$\mathrm{C}_{6} \mathrm{H}_{6}$} & [3] radialene & {$[\mathrm{T}-(\mathrm{T})]$} & -1.559 & -2.164 & & & & & \\
\hline & & {$[(\mathrm{Q})-\mathrm{T}]$} & 2.315 & 2.289 & & & & & \\
\hline \multirow[t]{2}{*}{$\mathrm{C}_{6} \mathrm{H}_{6}$} & benzene & {$[\mathrm{T}-(\mathrm{T})]$} & -1.881 & -2.564 & -2.630 & & & & \\
\hline & & {$[(\mathrm{Q})-\mathrm{T}]$} & 2.033 & 2.054 & & & & & \\
\hline \multirow[t]{2}{*}{$\mathrm{C}_{6} \mathrm{H}_{6}$} & benzvalene & {$[\mathrm{T}-(\mathrm{T})]$} & -2.021 & -2.719 & & & & & \\
\hline & & {$[(\mathrm{Q})-\mathrm{T}]$} & 2.042 & 1.959 & & & & & \\
\hline \multirow[t]{2}{*}{$\mathrm{C}_{6} \mathrm{H}_{6}$} & Dewar benzene & {$[\mathrm{T}-(\mathrm{T})]$} & -1.956 & -2.619 & & & & & \\
\hline & & {$[(\mathrm{Q})-\mathrm{T}]$} & 2.159 & 2.078 & & & & & \\
\hline \multirow[t]{2}{*}{$\mathrm{C}_{6} \mathrm{H}_{6}$} & fulvene & {$[\mathrm{T}-(\mathrm{T})]$} & -1.749 & -2.405 & -2.467 & & & & \\
\hline & & {$[(\mathrm{Q})-\mathrm{T}]$} & 2.317 & 2.292 & & & & & \\
\hline \multirow[t]{2}{*}{$\mathrm{C}_{6} \mathrm{H}_{6}$} & prismane & {$[\mathrm{T}-(\mathrm{T})]$} & -1.973 & -2.725 & -2.785 & & & & \\
\hline & & {$[(\mathrm{Q})-\mathrm{T}]$} & 1.914 & 1.809 & & & & & \\
\hline
\end{tabular}


Table S11, contd.

\begin{tabular}{|c|c|c|c|c|c|c|c|c|c|}
\hline & Molecule & Term & $\left(2,3^{\text {tr }}\right)$ & $\overline{\mathbf{3}^{\prime}}$ & $\overline{3}$ & $\overline{\overline{4^{\prime}}}$ & $\overline{4}$ & $\overline{\overline{5}^{\prime}}$ & $\overline{5}$ \\
\hline \multirow[t]{2}{*}{$\mathrm{C}_{6} \mathrm{H}_{8}$} & 1,3-cyclohexadiene & {$[\mathrm{T}-(\mathrm{T})]$} & -1.492 & & & & & & \\
\hline & & {$[(\mathrm{Q})-\mathrm{T}]$} & 1.800 & & & & & & \\
\hline \multirow[t]{2}{*}{$\mathrm{C}_{6} \mathrm{H}_{8}$} & 1,4-cyclohexadiene & {$[\mathrm{T}-(\mathrm{T})]$} & -1.447 & -2.028 & -2.106 & & & & \\
\hline & & {$[(\mathrm{Q})-\mathrm{T}]$} & 1.674 & 1.575 & & & & & \\
\hline \multirow[t]{2}{*}{$\mathrm{C}_{6} \mathrm{H}_{8}$} & trans-1,3,5-hexatriene & {$[\mathrm{T}-(\mathrm{T})]$} & -1.283 & & & & & & \\
\hline & & {$[(\mathrm{Q})-\mathrm{T}]$} & 1.927 & & & & & & \\
\hline \multirow[t]{2}{*}{$\mathrm{C}_{6} \mathrm{H}_{10}$} & cyclohexene & {$[\mathrm{T}-(\mathrm{T})]$} & -1.311 & & & & & & \\
\hline & & {$[(\mathrm{Q})-\mathrm{T}]$} & 1.425 & & & & & & \\
\hline \multirow[t]{2}{*}{$\mathrm{C}_{6} \mathrm{H}_{12}$} & cyclohexane & {$[\mathrm{T}-(\mathrm{T})]$} & -1.153 & & & & & & \\
\hline & & {$[(\mathrm{Q})-\mathrm{T}]$} & 1.169 & & & & & & \\
\hline \multirow[t]{2}{*}{$\mathrm{C}_{7} \mathrm{H}_{4}$} & 3,3-diethynylcyclopropene & {$[\mathrm{T}-(\mathrm{T})]$} & -2.565 & & & & & & \\
\hline & & {$[(\mathrm{Q})-\mathrm{T}]$} & 3.086 & & & & & & \\
\hline \multirow[t]{2}{*}{$\mathrm{C}_{7} \mathrm{H}_{4}$} & heptahexaene & {$[\mathrm{T}-(\mathrm{T})]$} & -1.962 & & & & & & \\
\hline & & {$[(\mathrm{Q})-\mathrm{T}]$} & 3.963 & & & & & & \\
\hline \multirow[t]{2}{*}{$\mathrm{C}_{7} \mathrm{H}_{6}$} & 1,1-diethynylcyclopropane & {$[\mathrm{T}-(\mathrm{T})]$} & -2.306 & & & & & & \\
\hline & & {$[(\mathrm{Q})-\mathrm{T}]$} & 2.724 & & & & & & \\
\hline \multirow[t]{2}{*}{$\mathrm{C}_{7} \mathrm{H}_{6}$} & 1,3,5-norcaratriene & {$[\mathrm{T}-(\mathrm{T})]$} & -2.554 & & & & & & \\
\hline & & {$[(\mathrm{Q})-\mathrm{T}]$} & 2.674 & & & & & & \\
\hline \multirow[t]{2}{*}{$\mathrm{C}_{7} \mathrm{H}_{6}$} & fulvenallene & {$[\mathrm{T}-(\mathrm{T})]$} & -2.156 & & & & & & \\
\hline & & {$[(\mathrm{Q})-\mathrm{T}]$} & 2.808 & & & & & & \\
\hline \multirow[t]{2}{*}{$\mathrm{C}_{7} \mathrm{H}_{6}$} & spiro[2.4]hepta-1,4,6-triene & {$[\mathrm{T}-(\mathrm{T})]$} & -2.408 & & & & & & \\
\hline & & {$[(\mathrm{Q})-\mathrm{T}]$} & 2.746 & & & & & & \\
\hline \multirow[t]{2}{*}{$\mathrm{C}_{7} \mathrm{H}_{8}$} & 1,3,5-cycloheptatriene & {$[\mathrm{T}-(\mathrm{T})]$} & -2.082 & & & & & & \\
\hline & & {$[(\mathrm{Q})-\mathrm{T}]$} & 2.536 & & & & & & \\
\hline \multirow[t]{2}{*}{$\mathrm{C}_{7} \mathrm{H}_{8}$} & 2,5-norbornadiene & {$[\mathrm{T}-(\mathrm{T})]$} & -2.223 & & & & & & \\
\hline & & {$[(\mathrm{Q})-\mathrm{T}]$} & 2.375 & & & & & & \\
\hline
\end{tabular}


Table S11, contd.

\begin{tabular}{|c|c|c|c|c|c|c|c|c|c|}
\hline & Molecule & Term & $\left(2,3^{\text {tr }}\right)$ & $\overline{\mathbf{3}^{\prime}}$ & $\overline{3}$ & $\overline{\overline{4^{\prime}}}$ & $\overline{4}$ & $\overline{\overline{5^{\prime}}}$ & $\overline{5}$ \\
\hline \multirow[t]{2}{*}{$\mathrm{C}_{7} \mathrm{H}_{8}$} & norcaradiene & {$[\mathrm{T}-(\mathrm{T})]$} & -2.107 & & & & & & \\
\hline & & {$[(\mathrm{Q})-\mathrm{T}]$} & 2.385 & & & & & & \\
\hline \multirow[t]{2}{*}{$\mathrm{C}_{7} \mathrm{H}_{8}$} & quadricyclane & {$[\mathrm{T}-(\mathrm{T})]$} & -2.225 & & & & & & \\
\hline & & {$[(\mathrm{Q})-\mathrm{T}]$} & 2.042 & & & & & & \\
\hline \multirow[t]{2}{*}{$\mathrm{C}_{7} \mathrm{H}_{8}$} & spiro[2.4]hepta-4,6-diene & {$[\mathrm{T}-(\mathrm{T})]$} & -2.158 & & & & & & \\
\hline & & {$[(\mathrm{Q})-\mathrm{T}]$} & 2.374 & & & & & & \\
\hline \multirow[t]{2}{*}{$\mathrm{C}_{8} \mathrm{H}_{2}$} & 1,3,5,7-octatetrayne & {$[\mathrm{T}-(\mathrm{T})]$} & -3.397 & & & & & & \\
\hline & & {$[(\mathrm{Q})-\mathrm{T}]$} & 5.071 & & & & & & \\
\hline \multirow[t]{2}{*}{$\mathrm{C}_{8} \mathrm{H}_{4}$} & 1,2,3,5,6,7-cyclooctahexaene & {$[\mathrm{T}-(\mathrm{T})]$} & -2.502 & & & & & & \\
\hline & & {$[(\mathrm{Q})-\mathrm{T}]$} & 4.717 & & & & & & \\
\hline \multirow[t]{2}{*}{$\mathrm{C}_{8} \mathrm{H}_{4}$} & 1,5-cyclooctadiene-3,7-diyne & {$[\mathrm{T}-(\mathrm{T})]$} & -3.066 & & & & & & \\
\hline & & {$[(\mathrm{Q})-\mathrm{T}]$} & 4.490 & & & & & & \\
\hline \multirow[t]{2}{*}{$\mathrm{C}_{8} \mathrm{H}_{4}$} & octaheptaene & {$[\mathrm{T}-(\mathrm{T})]$} & -2.257 & & & & & & \\
\hline & & {$[(\mathrm{Q})-\mathrm{T}]$} & 4.980 & & & & & & \\
\hline \multirow[t]{2}{*}{$\mathrm{C}_{8} \mathrm{H}_{6}$} & benzocyclobutadiene & {$[\mathrm{T}-(\mathrm{T})]$} & -2.795 & & & & & & \\
\hline & & {$[(\mathrm{Q})-\mathrm{T}]$} & 3.316 & & & & & & \\
\hline \multirow[t]{2}{*}{$\mathrm{C}_{8} \mathrm{H}_{6}$} & bis(cyclopropa)benzene & {$[\mathrm{T}-(\mathrm{T})]$} & -3.268 & & & & & & \\
\hline & & {$[(\mathrm{Q})-\mathrm{T}]$} & 3.336 & & & & & & \\
\hline \multirow[t]{2}{*}{$\mathrm{C}_{8} \mathrm{H}_{6}$} & pentalene & {$[\mathrm{T}-(\mathrm{T})]$} & -2.746 & & & & & & \\
\hline & & {$[(\mathrm{Q})-\mathrm{T}]$} & 3.698 & & & & & & \\
\hline \multirow[t]{2}{*}{$\mathrm{C}_{8} \mathrm{H}_{8}$} & 1,3,5,7-cyclooctatetraene & {$[\mathrm{T}-(\mathrm{T})]$} & -2.307 & & & & & & \\
\hline & & {$[(\mathrm{Q})-\mathrm{T}]$} & 2.873 & & & & & & \\
\hline \multirow[t]{2}{*}{$\mathrm{C}_{8} \mathrm{H}_{8}$} & benzocyclobutene & {$[\mathrm{T}-(\mathrm{T})]$} & -2.684 & & & & & & \\
\hline & & {$[(\mathrm{Q})-\mathrm{T}]$} & 2.749 & & & & & & \\
\hline \multirow[t]{2}{*}{$\mathrm{C}_{8} \mathrm{H}_{8}$} & cubane & {$[\mathrm{T}-(\mathrm{T})]$} & -2.562 & & & & & & \\
\hline & & {$[(\mathrm{Q})-\mathrm{T}]$} & 2.502 & & & & & & \\
\hline
\end{tabular}


Table S11, contd.

\begin{tabular}{|c|c|c|c|c|c|c|c|c|c|}
\hline & Molecule & Term & $\left(2,3^{t r}\right)$ & $\overline{\overline{3^{\prime}}}$ & $\overline{3}$ & $\overline{\overline{4^{\prime}}}$ & $\overline{4}$ & $\overline{\overline{5}^{\prime}}$ & $\overline{5}$ \\
\hline \multirow[t]{2}{*}{$\mathrm{C}_{8} \mathrm{H}_{8}$} & para-xylylene & {$[\mathrm{T}-(\mathrm{T})]$} & -2.085 & & & & & & \\
\hline & & {$[(\mathrm{Q})-\mathrm{T}]$} & 3.197 & & & & & & \\
\hline \multirow[t]{2}{*}{$\mathrm{C}_{10} \mathrm{H}_{4}$} & tetraethynylethene & {$[\mathrm{T}-(\mathrm{T})]$} & -3.844 & & & & & & \\
\hline & & {$[(\mathrm{Q})-\mathrm{T}]$} & 5.223 & & & & & & \\
\hline \multirow[t]{2}{*}{$\mathrm{C}_{10} \mathrm{H}_{8}$} & naphthalene & {$[\mathrm{T}-(\mathrm{T})]$} & -3.643 & & & & & & \\
\hline & & {$[(\mathrm{Q})-\mathrm{T}]$} & 3.942 & & & & & & \\
\hline \multirow[t]{2}{*}{$\mathrm{C}_{10} \mathrm{H}_{8}$} & pentafulvalene & {$[\mathrm{T}-(\mathrm{T})]$} & -3.385 & & & & & & \\
\hline & & {$[(\mathrm{Q})-\mathrm{T}]$} & 4.432 & & & & & & \\
\hline
\end{tabular}

${ }^{a}$ Basis sets are defined by their cardinal number as given in the header row (notation as in Sec 3). See text for further details and for definitions of terms. 
Table S12: Higher order electron correlation contributions to bond separation energies $(\mathrm{kcal} / \mathrm{mol} \text {, non-extrapolated })^{a}$

\begin{tabular}{|c|c|c|c|c|c|c|c|c|}
\hline & Molecule & Term & 2 & $3^{t r}$ & $3 \prime$ & 3 & $4^{\prime}$ & 4 \\
\hline \multirow[t]{6}{*}{$\mathrm{C}_{3} \mathrm{H}_{4}$} & allene & {$[\mathrm{T}-(\mathrm{T})]$} & -0.029 & -0.018 & -0.024 & -0.018 & -0.019 & -0.018 \\
\hline & & {$[(\mathrm{Q})-\mathrm{T}]$} & 0.037 & & 0.054 & 0.051 & 0.058 & \\
\hline & & {$[\mathrm{Q}-(\mathrm{Q})]$} & -0.014 & & & & & \\
\hline & & {$[(\mathrm{P})-\mathrm{Q}]$} & -0.002 & & & & & \\
\hline & & {$[\mathrm{T}-(\mathrm{T})]^{\text {core }}$} & 0.001 & & & -0.001 & & \\
\hline & & {$[(\mathrm{Q})-\mathrm{T}]^{\text {core }}$} & 0.002 & & & 0.002 & & \\
\hline \multirow[t]{6}{*}{$\mathrm{C}_{3} \mathrm{H}_{4}$} & cyclopropene & {$[\mathrm{T}-(\mathrm{T})]$} & -0.149 & -0.181 & -0.193 & -0.184 & -0.193 & -0.190 \\
\hline & & {$[(\mathrm{Q})-\mathrm{T}]$} & 0.188 & & 0.220 & 0.214 & 0.227 & \\
\hline & & {$[\mathrm{Q}-(\mathrm{Q})]$} & -0.035 & & & & & \\
\hline & & {$[(\mathrm{P})-\mathrm{Q}]$} & 0.025 & & & & & \\
\hline & & {$[\mathrm{T}-(\mathrm{T})]^{\text {core }}$} & -0.001 & & & 0.000 & & \\
\hline & & {$[(\mathrm{Q})-\mathrm{T}]^{\text {core }}$} & 0.002 & & & 0.005 & & \\
\hline \multirow[t]{5}{*}{$\mathrm{C}_{3} \mathrm{H}_{4}$} & propyne & {$[\mathrm{T}-(\mathrm{T})]$} & -0.048 & -0.039 & -0.037 & -0.036 & -0.034 & \\
\hline & & {$[(\mathrm{Q})-\mathrm{T}]$} & 0.028 & & 0.036 & 0.035 & 0.037 & \\
\hline & & {$[\mathrm{Q}-(\mathrm{Q})]$} & -0.017 & & & & & \\
\hline & & {$[\mathrm{T}-(\mathrm{T})]^{\text {core }}$} & 0.002 & & & 0.002 & & \\
\hline & & {$[(\mathrm{Q})-\mathrm{T}]^{\text {core }}$} & 0.000 & & & & & \\
\hline \multirow[t]{4}{*}{$\mathrm{C}_{3} \mathrm{H}_{6}$} & cyclopropane & {$[\mathrm{T}-(\mathrm{T})]$} & -0.065 & -0.082 & -0.082 & -0.079 & -0.082 & \\
\hline & & {$[(\mathrm{Q})-\mathrm{T}]$} & 0.080 & & 0.092 & 0.089 & 0.097 & \\
\hline & & {$[\mathrm{T}-(\mathrm{T})]^{\text {core }}$} & 0.001 & & & 0.002 & & \\
\hline & & {$[(\mathrm{Q})-\mathrm{T}]^{\text {core }}$} & 0.000 & & & & & \\
\hline \multirow[t]{4}{*}{$\mathrm{C}_{3} \mathrm{H}_{6}$} & propene & {$[\mathrm{T}-(\mathrm{T})]$} & -0.038 & -0.038 & -0.038 & -0.037 & & \\
\hline & & {$[(\mathrm{Q})-\mathrm{T}]$} & 0.035 & & 0.044 & & & \\
\hline & & {$[\mathrm{T}-(\mathrm{T})]^{\text {core }}$} & 0.000 & & & & & \\
\hline & & {$[(\mathrm{Q})-\mathrm{T}]^{\text {core }}$} & 0.001 & & & & & \\
\hline \multirow[t]{4}{*}{$\mathrm{C}_{3} \mathrm{H}_{8}$} & propane & {$[\mathrm{T}-(\mathrm{T})]$} & -0.021 & -0.022 & -0.025 & -0.025 & -0.027 & \\
\hline & & {$[(\mathrm{Q})-\mathrm{T}]$} & 0.014 & & 0.021 & & & \\
\hline & & {$[\mathrm{T}-(\mathrm{T})]^{\text {core }}$} & 0.000 & & & & & \\
\hline & & {$[(\mathrm{Q})-\mathrm{T}]^{\text {core }}$} & 0.000 & & & & & \\
\hline \multirow[t]{2}{*}{$\mathrm{C}_{4} \mathrm{H}_{2}$} & 1,3-butadiyne & {$[\mathrm{T}-(\mathrm{T})]$} & -0.200 & -0.192 & -0.220 & -0.207 & -0.213 & -0.210 \\
\hline & & {$[(\mathrm{Q})-\mathrm{T}]$} & 0.311 & & 0.385 & 0.376 & 0.394 & 0.392 \\
\hline
\end{tabular}


Table S12, contd.

\begin{tabular}{|c|c|c|c|c|c|c|c|c|}
\hline & Molecule & Term & 2 & $3^{t r}$ & 3 & 3 & $4^{\prime}$ & 4 \\
\hline & & {$[\mathrm{Q}-(\mathrm{Q})]$} & -0.076 & & & & & \\
\hline & & {$[(\mathrm{P})-\mathrm{Q}]$} & 0.020 & & & & & \\
\hline & & {$[\mathrm{T}-(\mathrm{T})]^{\text {core }}$} & 0.002 & & & 0.007 & & \\
\hline & & {$[(\mathrm{Q})-\mathrm{T}]^{\text {core }}$} & 0.004 & & & 0.005 & & \\
\hline \multirow[t]{5}{*}{$\mathrm{C}_{4} \mathrm{H}_{4}$} & bicyclo[1.1.0]but-1(3)-ene & {$[\mathrm{T}-(\mathrm{T})]$} & -0.405 & -0.489 & -0.527 & -0.510 & & \\
\hline & & {$[(\mathrm{Q})-\mathrm{T}]$} & 1.001 & & 1.145 & & & \\
\hline & & {$[\mathrm{Q}-(\mathrm{Q})]$} & -0.214 & & & & & \\
\hline & & {$[\mathrm{T}-(\mathrm{T})]^{\text {core }}$} & -0.001 & & & & & \\
\hline & & {$[(\mathrm{Q})-\mathrm{T}]^{\text {core }}$} & 0.015 & & & & & \\
\hline \multirow[t]{5}{*}{$\mathrm{C}_{4} \mathrm{H}_{4}$} & butatriene & {$[\mathrm{T}-(\mathrm{T})]$} & -0.059 & -0.053 & -0.073 & -0.057 & -0.068 & \\
\hline & & {$[(\mathrm{Q})-\mathrm{T}]$} & 0.275 & & 0.368 & 0.360 & 0.387 & \\
\hline & & {$[\mathrm{Q}-(\mathrm{Q})]$} & -0.072 & & & & & \\
\hline & & {$[\mathrm{T}-(\mathrm{T})]^{\text {core }}$} & 0.003 & & & & & \\
\hline & & {$[(\mathrm{Q})-\mathrm{T}]^{\text {core }}$} & 0.008 & & & & & \\
\hline \multirow[t]{5}{*}{$\mathrm{C}_{4} \mathrm{H}_{4}$} & cyclobutadiene & {$[\mathrm{T}-(\mathrm{T})]$} & 0.032 & -0.043 & -0.088 & -0.073 & -0.100 & -0.097 \\
\hline & & {$[(\mathrm{Q})-\mathrm{T}]$} & 0.483 & & 0.595 & 0.587 & 0.616 & \\
\hline & & {$[\mathrm{Q}-(\mathrm{Q})]$} & -0.096 & & & & & \\
\hline & & {$[\mathrm{T}-(\mathrm{T})]^{\text {core }}$} & 0.000 & & & & & \\
\hline & & {$[(\mathrm{Q})-\mathrm{T}]^{\text {core }}$} & 0.011 & & & & & \\
\hline \multirow[t]{4}{*}{$\mathrm{C}_{4} \mathrm{H}_{4}$} & ethynylethene & {$[\mathrm{T}-(\mathrm{T})]$} & -0.111 & -0.110 & -0.122 & -0.114 & & \\
\hline & & {$[(\mathrm{Q})-\mathrm{T}]$} & 0.155 & & 0.201 & & & \\
\hline & & {$[\mathrm{T}-(\mathrm{T})]^{\text {core }}$} & 0.002 & & & & & \\
\hline & & {$[(\mathrm{Q})-\mathrm{T}]^{\text {core }}$} & 0.004 & & & & & \\
\hline \multirow[t]{5}{*}{$\mathrm{C}_{4} \mathrm{H}_{4}$} & methylenecyclopropene & {$[\mathrm{T}-(\mathrm{T})]$} & -0.240 & -0.251 & -0.271 & -0.252 & & \\
\hline & & {$[(\mathrm{Q})-\mathrm{T}]$} & 0.212 & & 0.258 & & & \\
\hline & & {$[\mathrm{Q}-(\mathrm{Q})]$} & -0.040 & & & & & \\
\hline & & {$[\mathrm{T}-(\mathrm{T})]^{\text {core }}$} & 0.000 & & & & & \\
\hline & & {$[(\mathrm{Q})-\mathrm{T}]^{\text {core }}$} & 0.005 & & & & & \\
\hline \multirow[t]{4}{*}{$\mathrm{C}_{4} \mathrm{H}_{4}$} & tetrahedrane & {$[\mathrm{T}-(\mathrm{T})]$} & -0.316 & -0.388 & -0.436 & -0.412 & -0.442 & \\
\hline & & {$[(\mathrm{Q})-\mathrm{T}]$} & 0.349 & & 0.446 & 0.429 & & \\
\hline & & {$[\mathrm{T}-(\mathrm{T})]^{\text {core }}$} & 0.003 & & & & & \\
\hline & & {$[(\mathrm{Q})-\mathrm{T}]^{\text {core }}$} & 0.001 & & & & & \\
\hline
\end{tabular}


Table S12, contd.

\begin{tabular}{|c|c|c|c|c|c|c|c|c|}
\hline & Molecule & Term & 2 & $3^{t r}$ & $3 \prime$ & 3 & $44^{\prime}$ & 4 \\
\hline \multirow[t]{4}{*}{$\mathrm{C}_{4} \mathrm{H}_{6}$} & 1,2-butadiene & {$[\mathrm{T}-(\mathrm{T})]$} & -0.099 & -0.091 & -0.101 & -0.094 & & \\
\hline & & {$[(\mathrm{Q})-\mathrm{T}]$} & 0.092 & & 0.123 & & & \\
\hline & & {$[\mathrm{T}-(\mathrm{T})]^{\text {core }}$} & 0.001 & & & & & \\
\hline & & {$[(\mathrm{Q})-\mathrm{T}]^{\text {core }}$} & 0.003 & & & & & \\
\hline \multirow[t]{4}{*}{$\mathrm{C}_{4} \mathrm{H}_{6}$} & 1,3-butadiene & {$[\mathrm{T}-(\mathrm{T})]$} & -0.060 & -0.063 & -0.074 & -0.069 & & \\
\hline & & {$[(\mathrm{Q})-\mathrm{T}]$} & 0.134 & & 0.179 & & & \\
\hline & & {$[\mathrm{T}-(\mathrm{T})]^{\text {core }}$} & 0.000 & & & & & \\
\hline & & {$[(\mathrm{Q})-\mathrm{T}]^{\text {core }}$} & 0.004 & & & & & \\
\hline \multirow[t]{4}{*}{$\mathrm{C}_{4} \mathrm{H}_{6}$} & 2-butyne & {$[\mathrm{T}-(\mathrm{T})]$} & -0.102 & -0.086 & -0.086 & -0.082 & & \\
\hline & & {$[(\mathrm{Q})-\mathrm{T}]$} & 0.063 & & 0.081 & & & \\
\hline & & {$[\mathrm{T}-(\mathrm{T})]^{\text {core }}$} & 0.003 & & & & & \\
\hline & & {$[(\mathrm{Q})-\mathrm{T}]^{\text {core }}$} & 0.000 & & & & & \\
\hline \multirow[t]{4}{*}{$\mathrm{C}_{4} \mathrm{H}_{6}$} & bicyclo[1.1.0]butane & {$[\mathrm{T}-(\mathrm{T})]$} & -0.224 & -0.270 & -0.290 & -0.280 & & \\
\hline & & {$[(\mathrm{Q})-\mathrm{T}]$} & 0.253 & & 0.309 & & & \\
\hline & & {$[\mathrm{T}-(\mathrm{T})]^{\text {core }}$} & -0.001 & & & & & \\
\hline & & {$[(\mathrm{Q})-\mathrm{T}]^{\text {core }}$} & 0.002 & & & & & \\
\hline \multirow[t]{4}{*}{$\mathrm{C}_{4} \mathrm{H}_{6}$} & cyclobutene & {$[\mathrm{T}-(\mathrm{T})]$} & -0.174 & -0.199 & -0.219 & -0.212 & & \\
\hline & & {$[(\mathrm{Q})-\mathrm{T}]$} & 0.184 & & 0.222 & & & \\
\hline & & {$[\mathrm{T}-(\mathrm{T})]^{\text {core }}$} & -0.003 & & & & & \\
\hline & & {$[(\mathrm{Q})-\mathrm{T}]^{\text {core }}$} & 0.003 & & & & & \\
\hline \multirow[t]{4}{*}{$\mathrm{C}_{4} \mathrm{H}_{6}$} & methylenecyclopropane & {$[\mathrm{T}-(\mathrm{T})]$} & -0.133 & -0.151 & -0.151 & -0.143 & & \\
\hline & & {$[(\mathrm{Q})-\mathrm{T}]$} & 0.149 & & 0.176 & & & \\
\hline & & {$[\mathrm{T}-(\mathrm{T})]^{\text {core }}$} & 0.001 & & & & & \\
\hline & & {$[(\mathrm{Q})-\mathrm{T}]^{\text {core }}$} & 0.002 & & & & & \\
\hline \multirow[t]{4}{*}{$\mathrm{C}_{4} \mathrm{H}_{8}$} & cyclobutane & {$[\mathrm{T}-(\mathrm{T})]$} & -0.097 & -0.119 & -0.128 & -0.126 & & \\
\hline & & {$[(\mathrm{Q})-\mathrm{T}]$} & 0.098 & & 0.120 & & & \\
\hline & & {$[\mathrm{T}-(\mathrm{T})]^{\text {core }}$} & -0.001 & & & & & \\
\hline & & {$[(\mathrm{Q})-\mathrm{T}]^{\text {core }}$} & 0.001 & & & & & \\
\hline \multirow[t]{4}{*}{$\mathrm{C}_{4} \mathrm{H}_{8}$} & isobutene & {$[\mathrm{T}-(\mathrm{T})]$} & -0.101 & -0.101 & -0.107 & -0.106 & & \\
\hline & & {$[(\mathrm{Q})-\mathrm{T}]$} & 0.076 & & 0.101 & & & \\
\hline & & {$[\mathrm{T}-(\mathrm{T})]^{\text {core }}$} & 0.000 & & & & & \\
\hline & & {$[(\mathrm{Q})-\mathrm{T}]^{\text {core }}$} & 0.002 & & & & & \\
\hline
\end{tabular}


Table S12, contd.

\begin{tabular}{|c|c|c|c|c|c|c|c|c|}
\hline & Molecule & Term & 2 & $3^{t r}$ & 3, & 3 & $4^{\prime}$ & 4 \\
\hline \multirow[t]{2}{*}{$\mathrm{C}_{4} \mathrm{H}_{10}$} & isobutane & {$[\mathrm{T}-(\mathrm{T})]$} & -0.068 & -0.070 & & & & \\
\hline & & {$[(\mathrm{Q})-\mathrm{T}]$} & 0.041 & & & & & \\
\hline \multirow[t]{4}{*}{$\mathrm{C}_{4} \mathrm{H}_{10}$} & $n$-butane & {$[\mathrm{T}-(\mathrm{T})]$} & -0.049 & -0.051 & -0.058 & -0.058 & & \\
\hline & & {$[(\mathrm{Q})-\mathrm{T}]$} & 0.031 & & 0.047 & & & \\
\hline & & {$[\mathrm{T}-(\mathrm{T})]^{\text {core }}$} & -0.001 & & & & & \\
\hline & & {$[(\mathrm{Q})-\mathrm{T}]^{\text {core }}$} & 0.001 & & & & & \\
\hline \multirow[t]{4}{*}{$\mathrm{C}_{5} \mathrm{H}_{4}$} & 1,4-pentadiyne & {$[\mathrm{T}-(\mathrm{T})]$} & -0.171 & -0.160 & -0.172 & & & \\
\hline & & {$[(\mathrm{Q})-\mathrm{T}]$} & 0.132 & & 0.163 & & & \\
\hline & & {$[\mathrm{T}-(\mathrm{T})]^{\text {core }}$} & 0.003 & & & & & \\
\hline & & {$[(\mathrm{Q})-\mathrm{T}]^{\text {core }}$} & 0.003 & & & & & \\
\hline \multirow[t]{4}{*}{$\mathrm{C}_{5} \mathrm{H}_{4}$} & pentatetraene & {$[\mathrm{T}-(\mathrm{T})]$} & -0.187 & -0.178 & -0.222 & -0.196 & & \\
\hline & & {$[(\mathrm{Q})-\mathrm{T}]$} & 0.547 & & 0.709 & & & \\
\hline & & {$[\mathrm{T}-(\mathrm{T})]^{\text {core }}$} & 0.004 & & & & & \\
\hline & & {$[(\mathrm{Q})-\mathrm{T}]^{\text {core }}$} & 0.012 & & & & & \\
\hline \multirow[t]{4}{*}{$\mathrm{C}_{5} \mathrm{H}_{4}$} & spiropentadiene & {$[\mathrm{T}-(\mathrm{T})]$} & -0.468 & -0.535 & -0.575 & -0.543 & & \\
\hline & & {$[(\mathrm{Q})-\mathrm{T}]$} & 0.496 & & 0.597 & & & \\
\hline & & {$[\mathrm{T}-(\mathrm{T})]^{\text {core }}$} & -0.003 & & & & & \\
\hline & & {$[(\mathrm{Q})-\mathrm{T}]^{\text {core }}$} & 0.011 & & & & & \\
\hline \multirow[t]{2}{*}{$\mathrm{C}_{5} \mathrm{H}_{6}$} & [1.1.1]propellane & {$[\mathrm{T}-(\mathrm{T})]$} & -0.621 & -0.712 & -0.766 & -0.746 & & \\
\hline & & {$[(\mathrm{Q})-\mathrm{T}]$} & 0.748 & & 0.902 & & & \\
\hline \multirow[t]{2}{*}{$\mathrm{C}_{5} \mathrm{H}_{6}$} & 1,3-cyclopentadiene & {$[\mathrm{T}-(\mathrm{T})]$} & -0.326 & -0.348 & -0.382 & -0.370 & & \\
\hline & & {$[(\mathrm{Q})-\mathrm{T}]$} & 0.321 & & 0.403 & & & \\
\hline \multirow[t]{2}{*}{$\mathrm{C}_{5} \mathrm{H}_{6}$} & 3-methylenecyclobutene & {$[\mathrm{T}-(\mathrm{T})]$} & -0.272 & -0.296 & & & & \\
\hline & & {$[(\mathrm{Q})-\mathrm{T}]$} & 0.307 & & & & & \\
\hline \multirow[t]{2}{*}{$\mathrm{C}_{5} \mathrm{H}_{6}$} & bicyclo[2.1.0]pent-2-ene & {$[\mathrm{T}-(\mathrm{T})]$} & -0.374 & -0.432 & & & & \\
\hline & & {$[(\mathrm{Q})-\mathrm{T}]$} & 0.415 & & & & & \\
\hline \multirow[t]{2}{*}{$\mathrm{C}_{5} \mathrm{H}_{6}$} & cyclopropylacetylene & {$[\mathrm{T}-(\mathrm{T})]$} & -0.222 & -0.234 & & & & \\
\hline & & {$[(\mathrm{Q})-\mathrm{T}]$} & 0.195 & & & & & \\
\hline
\end{tabular}


Table S12, contd.

\begin{tabular}{|c|c|c|c|c|c|c|c|c|}
\hline & Molecule & Term & 2 & $3^{t r}$ & 3, & 3 & $4^{\prime}$ & 4 \\
\hline \multirow[t]{2}{*}{$\mathrm{C}_{5} \mathrm{H}_{8}$} & 1,4-pentadiene & {$[\mathrm{T}-(\mathrm{T})]$} & -0.147 & -0.151 & & & & \\
\hline & & {$[(\mathrm{Q})-\mathrm{T}]$} & 0.127 & & & & & \\
\hline \multirow[t]{2}{*}{$\mathrm{C}_{5} \mathrm{H}_{8}$} & isoprene & {$[\mathrm{T}-(\mathrm{T})]$} & -0.148 & -0.153 & & & & \\
\hline & & {$[(\mathrm{Q})-\mathrm{T}]$} & 0.191 & & & & & \\
\hline \multirow[t]{2}{*}{$\mathrm{C}_{5} \mathrm{H}_{8}$} & spiropentane & {$[\mathrm{T}-(\mathrm{T})]$} & -0.235 & -0.269 & -0.516 & -0.261 & & \\
\hline & & {$[(\mathrm{Q})-\mathrm{T}]$} & 0.207 & & 0.648 & & & \\
\hline \multirow[t]{2}{*}{$\mathrm{C}_{5} \mathrm{H}_{10}$} & cyclopentane & {$[\mathrm{T}-(\mathrm{T})]$} & -0.148 & -0.161 & & & & \\
\hline & & {$[(\mathrm{Q})-\mathrm{T}]$} & 0.096 & & & & & \\
\hline \multirow[t]{2}{*}{$\mathrm{C}_{5} \mathrm{H}_{12}$} & neopentane & {$[\mathrm{T}-(\mathrm{T})]$} & -0.146 & -0.151 & & & & \\
\hline & & {$[(\mathrm{Q})-\mathrm{T}]$} & 0.080 & & & & & \\
\hline \multirow[t]{2}{*}{$\mathrm{C}_{5} \mathrm{H}_{12}$} & $n$-pentane & {$[\mathrm{T}-(\mathrm{T})]$} & -0.078 & -0.081 & & & & \\
\hline & & {$[(\mathrm{Q})-\mathrm{T}]$} & 0.049 & & & & & \\
\hline \multirow[t]{4}{*}{$\mathrm{C}_{6} \mathrm{H}_{2}$} & 1,3,5-hexatriyne & {$[\mathrm{T}-(\mathrm{T})]$} & -0.502 & -0.490 & -0.557 & -0.531 & & \\
\hline & & {$[(\mathrm{Q})-\mathrm{T}]$} & 0.779 & & 0.957 & & & \\
\hline & & {$[\mathrm{T}-(\mathrm{T})]^{\text {core }}$} & 0.005 & & & & & \\
\hline & & {$[(\mathrm{Q})-\mathrm{T}]^{\text {core }}$} & 0.009 & & & & & \\
\hline \multirow[t]{4}{*}{$\mathrm{C}_{6} \mathrm{H}_{4}$} & butalene & {$[\mathrm{T}-(\mathrm{T})]$} & -1.109 & -1.213 & -1.375 & & & \\
\hline & & {$[(\mathrm{Q})-\mathrm{T}]$} & 1.223 & & 1.515 & & & \\
\hline & & {$[\mathrm{T}-(\mathrm{T})]^{\text {core }}$} & -0.009 & & & & & \\
\hline & & {$[(\mathrm{Q})-\mathrm{T}]^{\text {core }}$} & 0.027 & & & & & \\
\hline \multirow[t]{2}{*}{$\mathrm{C}_{6} \mathrm{H}_{4}$} & cis-3-hexene-1,5-diyne & {$[\mathrm{T}-(\mathrm{T})]$} & -0.286 & -0.286 & & & & \\
\hline & & {$[(\mathrm{Q})-\mathrm{T}]$} & 0.396 & & & & & \\
\hline \multirow[t]{4}{*}{$\mathrm{C}_{6} \mathrm{H}_{4}$} & hexapentaene & {$[\mathrm{T}-(\mathrm{T})]$} & -0.207 & -0.210 & -0.282 & -0.244 & & \\
\hline & & {$[(\mathrm{Q})-\mathrm{T}]$} & 0.953 & & 1.224 & & & \\
\hline & & {$[\mathrm{T}-(\mathrm{T})]^{\text {core }}$} & 0.008 & & & & & \\
\hline & & {$[(\mathrm{Q})-\mathrm{T}]^{\text {core }}$} & 0.020 & & & & & \\
\hline \multirow[t]{2}{*}{$\mathrm{C}_{6} \mathrm{H}_{4}$} & ortho-benzyne & {$[\mathrm{T}-(\mathrm{T})]$} & -0.939 & -0.998 & -1.128 & -1.105 & & \\
\hline & & {$[(\mathrm{Q})-\mathrm{T}]$} & 1.142 & & 1.412 & & & \\
\hline
\end{tabular}


Table S12, contd.

\begin{tabular}{|c|c|c|c|c|c|c|c|c|}
\hline & Molecule & Term & 2 & $3^{t r}$ & 3 & 3 & $4^{\prime}$ & 4 \\
\hline \multirow[t]{2}{*}{$\mathrm{C}_{6} \mathrm{H}_{4}$} & trans-3-hexene-1,5-diyne & {$[\mathrm{T}-(\mathrm{T})]$} & -0.260 & -0.259 & -0.293 & -0.274 & & \\
\hline & & {$[(\mathrm{Q})-\mathrm{T}]$} & 0.380 & & 0.485 & & & \\
\hline \multirow[t]{4}{*}{$\mathrm{C}_{6} \mathrm{H}_{4}$} & triafulvalene & {$[\mathrm{T}-(\mathrm{T})]$} & -0.566 & -0.621 & -0.688 & -0.643 & & \\
\hline & & {$[(\mathrm{Q})-\mathrm{T}]$} & 0.589 & & 0.714 & & & \\
\hline & & {$[\mathrm{T}-(\mathrm{T})]^{\text {core }}$} & -0.002 & & & & & \\
\hline & & {$[(\mathrm{Q})-\mathrm{T}]^{\text {core }}$} & 0.013 & & & & & \\
\hline \multirow[t]{2}{*}{$\mathrm{C}_{6} \mathrm{H}_{6}$} & 2,4-hexadiyne & {$[\mathrm{T}-(\mathrm{T})]$} & -0.363 & -0.334 & -0.364 & -0.350 & & \\
\hline & & {$[(\mathrm{Q})-\mathrm{T}]$} & 0.407 & & 0.506 & & & \\
\hline \multirow[t]{2}{*}{$\mathrm{C}_{6} \mathrm{H}_{6}$} & 3,3'-bicyclopropenyl & {$[\mathrm{T}-(\mathrm{T})]$} & -0.493 & -0.561 & -0.606 & & & \\
\hline & & {$[(\mathrm{Q})-\mathrm{T}]$} & 0.492 & & 0.595 & & & \\
\hline \multirow[t]{2}{*}{$\mathrm{C}_{6} \mathrm{H}_{6}$} & 3,4-dimethylenecyclobutene & {$[\mathrm{T}-(\mathrm{T})]$} & -0.376 & -0.400 & -0.451 & & & \\
\hline & & {$[(\mathrm{Q})-\mathrm{T}]$} & 0.465 & & 0.598 & & & \\
\hline \multirow[t]{2}{*}{$\mathrm{C}_{6} \mathrm{H}_{6}$} & [3]radialene & {$[\mathrm{T}-(\mathrm{T})]$} & -0.257 & -0.285 & -0.318 & & & \\
\hline & & {$[(\mathrm{Q})-\mathrm{T}]$} & 0.579 & & 0.722 & & & \\
\hline \multirow[t]{3}{*}{$\mathrm{C}_{6} \mathrm{H}_{6}$} & benzene & {$[\mathrm{T}-(\mathrm{T})]$} & -0.649 & -0.642 & -0.715 & -0.695 & & \\
\hline & & {$[(\mathrm{Q})-\mathrm{T}]$} & 0.353 & & 0.489 & & & \\
\hline & & {$[\mathrm{T}-(\mathrm{T})]^{\text {core }}$} & -0.005 & & & & & \\
\hline \multirow[t]{2}{*}{$\mathrm{C}_{6} \mathrm{H}_{6}$} & benzvalene & {$[\mathrm{T}-(\mathrm{T})]$} & -0.590 & -0.660 & -0.719 & & & \\
\hline & & {$[(\mathrm{Q})-\mathrm{T}]$} & 0.532 & & 0.663 & & & \\
\hline \multirow[t]{2}{*}{$\mathrm{C}_{6} \mathrm{H}_{6}$} & Dewar benzene & {$[\mathrm{T}-(\mathrm{T})]$} & -0.559 & -0.623 & -0.683 & & & \\
\hline & & {$[(\mathrm{Q})-\mathrm{T}]$} & 0.540 & & 0.655 & & & \\
\hline \multirow[t]{2}{*}{$\mathrm{C}_{6} \mathrm{H}_{6}$} & fulvene & {$[\mathrm{T}-(\mathrm{T})]$} & -0.454 & -0.479 & -0.546 & -0.523 & & \\
\hline & & {$[(\mathrm{Q})-\mathrm{T}]$} & 0.581 & & 0.724 & & & \\
\hline \multirow[t]{2}{*}{$\mathrm{C}_{6} \mathrm{H}_{6}$} & prismane & {$[\mathrm{T}-(\mathrm{T})]$} & -0.495 & -0.577 & -0.651 & -0.618 & & \\
\hline & & {$[(\mathrm{Q})-\mathrm{T}]$} & 0.516 & & 0.646 & & & \\
\hline \multirow[t]{2}{*}{$\mathrm{C}_{6} \mathrm{H}_{8}$} & 1,3-cyclohexadiene & {$[\mathrm{T}-(\mathrm{T})]$} & -0.304 & -0.322 & & & & \\
\hline & & {$[(\mathrm{Q})-\mathrm{T}]$} & 0.313 & & & & & \\
\hline
\end{tabular}


Table S12, contd.

\begin{tabular}{|c|c|c|c|c|c|c|c|c|}
\hline & Molecule & Term & 2 & $3^{t r}$ & 3 & 3 & 4 & 4 \\
\hline \multirow[t]{2}{*}{$\mathrm{C}_{6} \mathrm{H}_{8}$} & 1,4-cyclohexadiene & {$[\mathrm{T}-(\mathrm{T})]$} & -0.285 & -0.290 & -0.306 & -0.297 & & \\
\hline & & {$[(\mathrm{Q})-\mathrm{T}]$} & 0.212 & & 0.270 & & & \\
\hline \multirow[t]{2}{*}{$\mathrm{C}_{6} \mathrm{H}_{8}$} & trans-1,3,5-hexatriene & {$[\mathrm{T}-(\mathrm{T})]$} & -0.134 & -0.144 & & & & \\
\hline & & {$[(\mathrm{Q})-\mathrm{T}]$} & 0.329 & & & & & \\
\hline \multirow[t]{2}{*}{$\mathrm{C}_{6} \mathrm{H}_{10}$} & cyclohexene & {$[\mathrm{T}-(\mathrm{T})]$} & -0.237 & -0.246 & & & & \\
\hline & & {$[(\mathrm{Q})-\mathrm{T}]$} & 0.160 & & & & & \\
\hline \multirow[t]{2}{*}{$\mathrm{C}_{6} \mathrm{H}_{12}$} & cyclohexane & {$[\mathrm{T}-(\mathrm{T})]$} & -0.180 & -0.185 & & & & \\
\hline & & {$[(\mathrm{Q})-\mathrm{T}]$} & 0.101 & & & & & \\
\hline \multirow[t]{2}{*}{$\mathrm{C}_{7} \mathrm{H}_{4}$} & 3,3-diethynylcyclopropene & {$[\mathrm{T}-(\mathrm{T})]$} & -0.595 & -0.622 & & & & \\
\hline & & {$[(\mathrm{Q})-\mathrm{T}]$} & 0.523 & & & & & \\
\hline \multirow[t]{2}{*}{$\mathrm{C}_{7} \mathrm{H}_{4}$} & heptahexaene & {$[\mathrm{T}-(\mathrm{T})]$} & -0.373 & -0.381 & & & & \\
\hline & & {$[(\mathrm{Q})-\mathrm{T}]$} & 1.380 & & & & & \\
\hline \multirow[t]{2}{*}{$\mathrm{C}_{7} \mathrm{H}_{6}$} & 1,1-diethynylcyclopropane & {$[\mathrm{T}-(\mathrm{T})]$} & -0.461 & -0.472 & & & & \\
\hline & & {$[(\mathrm{Q})-\mathrm{T}]$} & 0.380 & & & & & \\
\hline \multirow[t]{2}{*}{$\mathrm{C}_{7} \mathrm{H}_{6}$} & 1,3,5-norcaratriene & {$[\mathrm{T}-(\mathrm{T})]$} & -0.958 & -0.995 & & & & \\
\hline & & {$[(\mathrm{Q})-\mathrm{T}]$} & 0.667 & & & & & \\
\hline \multirow[t]{2}{*}{$\mathrm{C}_{7} \mathrm{H}_{6}$} & fulvenallene & {$[\mathrm{T}-(\mathrm{T})]$} & -0.613 & -0.634 & & & & \\
\hline & & {$[(\mathrm{Q})-\mathrm{T}]$} & 0.688 & & & & & \\
\hline \multirow[t]{2}{*}{$\mathrm{C}_{7} \mathrm{H}_{6}$} & spiro[2.4]hepta-1,4,6-triene & {$[\mathrm{T}-(\mathrm{T})]$} & -0.767 & -0.826 & & & & \\
\hline & & {$[(\mathrm{Q})-\mathrm{T}]$} & 0.725 & & & & & \\
\hline \multirow[t]{2}{*}{$\mathrm{C}_{7} \mathrm{H}_{8}$} & 1,3,5-cycloheptatriene & {$[\mathrm{T}-(\mathrm{T})]$} & -0.606 & -0.641 & & & & \\
\hline & & {$[(\mathrm{Q})-\mathrm{T}]$} & 0.617 & & & & & \\
\hline \multirow[t]{2}{*}{$\mathrm{C}_{7} \mathrm{H}_{8}$} & 2,5-norbornadiene & {$[\mathrm{T}-(\mathrm{T})]$} & -0.684 & -0.739 & & & & \\
\hline & & {$[(\mathrm{Q})-\mathrm{T}]$} & 0.574 & & & & & \\
\hline \multirow[t]{2}{*}{$\mathrm{C}_{7} \mathrm{H}_{8}$} & norcaradiene & {$[\mathrm{T}-(\mathrm{T})]$} & -0.592 & -0.635 & & & & \\
\hline & & {$[(\mathrm{Q})-\mathrm{T}]$} & 0.582 & & & & & \\
\hline
\end{tabular}


Table S12, contd.

\begin{tabular}{|c|c|c|c|c|c|c|c|c|}
\hline & Molecule & Term & 2 & $3^{t r}$ & 3 & 3 & $44^{\prime}$ & 4 \\
\hline \multirow[t]{2}{*}{$\mathrm{C}_{7} \mathrm{H}_{8}$} & quadricyclane & {$[\mathrm{T}-(\mathrm{T})]$} & -0.625 & -0.688 & & & & \\
\hline & & {$[(\mathrm{Q})-\mathrm{T}]$} & 0.479 & & & & & \\
\hline \multirow[t]{2}{*}{$\mathrm{C}_{7} \mathrm{H}_{8}$} & spiro[2.4]hepta-4,6-diene & {$[\mathrm{T}-(\mathrm{T})]$} & -0.631 & -0.680 & & & & \\
\hline & & {$[(\mathrm{Q})-\mathrm{T}]$} & 0.573 & & & & & \\
\hline \multirow[t]{2}{*}{$\mathrm{C}_{8} \mathrm{H}_{2}$} & 1,3,5,7-octatetrayne & {$[\mathrm{T}-(\mathrm{T})]$} & -0.863 & -0.848 & & & & \\
\hline & & {$[(\mathrm{Q})-\mathrm{T}]$} & 1.353 & & & & & \\
\hline \multirow[t]{2}{*}{$\mathrm{C}_{8} \mathrm{H}_{4}$} & 1,2,3,5,6,7-cyclooctahexaene & {$[\mathrm{T}-(\mathrm{T})]$} & -0.417 & -0.534 & & & & \\
\hline & & {$[(\mathrm{Q})-\mathrm{T}]$} & 1.783 & & & & & \\
\hline \multirow[t]{2}{*}{$\mathrm{C}_{8} \mathrm{H}_{4}$} & 1,5-cyclooctadiene-3,7-diyne & {$[\mathrm{T}-(\mathrm{T})]$} & -0.775 & -0.835 & & & & \\
\hline & & {$[(\mathrm{Q})-\mathrm{T}]$} & 1.361 & & & & & \\
\hline \multirow[t]{2}{*}{$\mathrm{C}_{8} \mathrm{H}_{4}$} & octaheptaene & {$[\mathrm{T}-(\mathrm{T})]$} & -0.382 & -0.406 & & & & \\
\hline & & {$[(\mathrm{Q})-\mathrm{T}]$} & 1.908 & & & & & \\
\hline \multirow[t]{2}{*}{$\mathrm{C}_{8} \mathrm{H}_{6}$} & benzocyclobutadiene & {$[\mathrm{T}-(\mathrm{T})]$} & -0.930 & -0.974 & & & & \\
\hline & & {$[(\mathrm{Q})-\mathrm{T}]$} & 0.895 & & & & & \\
\hline \multirow[t]{2}{*}{$\mathrm{C}_{8} \mathrm{H}_{6}$} & bis(cyclopropa)benzene & {$[\mathrm{T}-(\mathrm{T})]$} & -1.305 & -1.386 & & & & \\
\hline & & {$[(\mathrm{Q})-\mathrm{T}]$} & 0.997 & & & & & \\
\hline \multirow[t]{2}{*}{$\mathrm{C}_{8} \mathrm{H}_{6}$} & pentalene & {$[\mathrm{T}-(\mathrm{T})]$} & -0.849 & -0.908 & & & & \\
\hline & & {$[(\mathrm{Q})-\mathrm{T}]$} & 1.201 & & & & & \\
\hline \multirow[t]{2}{*}{$\mathrm{C}_{8} \mathrm{H}_{8}$} & 1,3,5,7-cyclooctatetraene & {$[\mathrm{T}-(\mathrm{T})]$} & -0.582 & -0.614 & & & & \\
\hline & & {$[(\mathrm{Q})-\mathrm{T}]$} & 0.601 & & & & & \\
\hline \multirow[t]{2}{*}{$\mathrm{C}_{8} \mathrm{H}_{8}$} & benzocyclobutene & {$[\mathrm{T}-(\mathrm{T})]$} & -0.960 & -0.980 & & & & \\
\hline & & {$[(\mathrm{Q})-\mathrm{T}]$} & 0.588 & & & & & \\
\hline \multirow[t]{2}{*}{$\mathrm{C}_{8} \mathrm{H}_{8}$} & cubane & {$[\mathrm{T}-(\mathrm{T})]$} & -0.631 & -0.721 & & & & \\
\hline & & {$[(\mathrm{Q})-\mathrm{T}]$} & 0.648 & & & & & \\
\hline \multirow[t]{2}{*}{$\mathrm{C}_{8} \mathrm{H}_{8}$} & para-xylylene & {$[\mathrm{T}-(\mathrm{T})]$} & -0.372 & -0.398 & & & & \\
\hline & & {$[(\mathrm{Q})-\mathrm{T}]$} & 0.860 & & & & & \\
\hline
\end{tabular}


Table S12, contd.

\begin{tabular}{|c|c|c|c|c|c|c|c|c|}
\hline & Molecule & Term & 2 & $3^{t r}$ & $3{ }^{\prime}$ & 3 & 4 ' & 4 \\
\hline \multirow[t]{2}{*}{$\mathrm{C}_{10} \mathrm{H}_{4}$} & tetraethynylethene & {$[\mathrm{T}-(\mathrm{T})]$} & -0.835 & -0.850 & & & & \\
\hline & & {$[(\mathrm{Q})-\mathrm{T}]$} & 1.050 & & & & & \\
\hline \multirow[t]{2}{*}{$\mathrm{C}_{10} \mathrm{H}_{8}$} & naphthalene & {$[\mathrm{T}-(\mathrm{T})]$} & -1.447 & -1.448 & & & & \\
\hline & & {$[(\mathrm{Q})-\mathrm{T}]$} & 0.972 & & & & & \\
\hline \multirow[t]{2}{*}{$\mathrm{C}_{10} \mathrm{H}_{8}$} & pentafulvalene & {$[\mathrm{T}-(\mathrm{T})]$} & -1.074 & -1.133 & & & & \\
\hline & & {$[(\mathrm{Q})-\mathrm{T}]$} & 1.364 & & & & & \\
\hline
\end{tabular}

${ }^{a}$ Basis sets are defined by their cardinal number as given in the header row (notation as in Sec 3). See text for further details and for definitions of terms. Note that bond separation energy contributions are zero by definition for all BSR prototypes (methane, ethane, ethylene, acetylene) and so not listed here. 
Table S13: Higher order electron correlation contributions to bond separation energies $(\mathrm{kcal} / \mathrm{mol} \text {, CBS-extrapolated })^{a}$

\begin{tabular}{|c|c|c|c|c|c|c|c|}
\hline & Molecule & Term & $\left(2,3^{t r}\right)$ & $\overline{3^{\prime}}$ & $\overline{3}$ & $\overline{\overline{4^{\prime}}}$ & $\overline{4}$ \\
\hline \multirow[t]{4}{*}{$\mathrm{C}_{3} \mathrm{H}_{4}$} & allene & {$[\mathrm{T}-(\mathrm{T})]$} & -0.007 & -0.021 & -0.013 & -0.020 & -0.019 \\
\hline & & {$[(\mathrm{Q})-\mathrm{T}]$} & 0.047 & 0.061 & 0.056 & 0.063 & \\
\hline & & {$[\mathrm{T}-(\mathrm{T})]^{\text {core }}$} & & & -0.001 & & \\
\hline & & {$[(\mathrm{Q})-\mathrm{T}]^{\text {core }}$} & & & 0.002 & & \\
\hline \multirow[t]{4}{*}{$\mathrm{C}_{3} \mathrm{H}_{4}$} & cyclopropene & {$[\mathrm{T}-(\mathrm{T})]$} & -0.213 & -0.212 & -0.199 & -0.199 & -0.195 \\
\hline & & {$[(\mathrm{Q})-\mathrm{T}]$} & 0.235 & 0.233 & 0.225 & 0.236 & \\
\hline & & {$[\mathrm{T}-(\mathrm{T})]^{\text {core }}$} & & & 0.000 & & \\
\hline & & {$[(\mathrm{Q})-\mathrm{T}]^{\text {core }}$} & & & 0.007 & & \\
\hline \multirow[t]{3}{*}{$\mathrm{C}_{3} \mathrm{H}_{4}$} & propyne & {$[\mathrm{T}-(\mathrm{T})]$} & -0.031 & -0.033 & -0.031 & -0.033 & \\
\hline & & {$[(\mathrm{Q})-\mathrm{T}]$} & 0.035 & 0.039 & 0.038 & 0.038 & \\
\hline & & {$[\mathrm{T}-(\mathrm{T})]^{\text {core }}$} & & & 0.003 & & \\
\hline \multirow[t]{3}{*}{$\mathrm{C}_{3} \mathrm{H}_{6}$} & cyclopropane & {$[\mathrm{T}-(\mathrm{T})]$} & -0.099 & -0.089 & -0.085 & -0.085 & \\
\hline & & {$[(\mathrm{Q})-\mathrm{T}]$} & 0.100 & 0.097 & 0.093 & 0.102 & \\
\hline & & {$[\mathrm{T}-(\mathrm{T})]^{\text {core }}$} & & & 0.002 & & \\
\hline \multirow[t]{2}{*}{$\mathrm{C}_{3} \mathrm{H}_{6}$} & propene & {$[\mathrm{T}-(\mathrm{T})]$} & -0.038 & -0.038 & -0.037 & & \\
\hline & & {$[(\mathrm{Q})-\mathrm{T}]$} & 0.043 & 0.048 & & & \\
\hline \multirow[t]{2}{*}{$\mathrm{C}_{3} \mathrm{H}_{8}$} & propane & {$[\mathrm{T}-(\mathrm{T})]$} & -0.023 & -0.027 & -0.026 & -0.029 & \\
\hline & & {$[(\mathrm{Q})-\mathrm{T}]$} & 0.018 & 0.024 & & & \\
\hline \multirow[t]{4}{*}{$\mathrm{C}_{4} \mathrm{H}_{2}$} & 1,3-butadiyne & {$[\mathrm{T}-(\mathrm{T})]$} & -0.183 & -0.228 & -0.210 & -0.218 & -0.212 \\
\hline & & {$[(\mathrm{Q})-\mathrm{T}]$} & 0.388 & 0.417 & 0.404 & 0.407 & 0.404 \\
\hline & & {$[\mathrm{T}-(\mathrm{T})]^{\text {core }}$} & & & 0.010 & & \\
\hline & & {$[(\mathrm{Q})-\mathrm{T}]^{\text {core }}$} & & & 0.006 & & \\
\hline \multirow[t]{2}{*}{$\mathrm{C}_{4} \mathrm{H}_{4}$} & bicyclo[1.1.0]but-1(3)-ene & {$[\mathrm{T}-(\mathrm{T})]$} & -0.573 & -0.579 & -0.554 & & \\
\hline & & {$[(\mathrm{Q})-\mathrm{T}]$} & 1.251 & 1.206 & & & \\
\hline \multirow[t]{2}{*}{$\mathrm{C}_{4} \mathrm{H}_{4}$} & butatriene & {$[\mathrm{T}-(\mathrm{T})]$} & -0.046 & -0.078 & -0.056 & -0.076 & \\
\hline & & {$[(\mathrm{Q})-\mathrm{T}]$} & 0.344 & 0.407 & 0.396 & 0.407 & \\
\hline \multirow[t]{2}{*}{$\mathrm{C}_{4} \mathrm{H}_{4}$} & cyclobutadiene & {$[\mathrm{T}-(\mathrm{T})]$} & -0.119 & -0.139 & -0.117 & -0.120 & -0.114 \\
\hline & & {$[(\mathrm{Q})-\mathrm{T}]$} & 0.603 & 0.642 & 0.632 & 0.638 & \\
\hline
\end{tabular}


Table S13, contd.

\begin{tabular}{|c|c|c|c|c|c|c|c|}
\hline & Molecule & Term & $\left(2,3^{t r}\right)$ & $\overline{3^{\prime}}$ & $\overline{3}$ & $\overline{4^{\prime}}$ & $\overline{4}$ \\
\hline \multirow[t]{2}{*}{$\mathrm{C}_{4} \mathrm{H}_{4}$} & ethynylethene & {$[\mathrm{T}-(\mathrm{T})]$} & -0.108 & -0.126 & -0.116 & & \\
\hline & & {$[(\mathrm{Q})-\mathrm{T}]$} & 0.194 & 0.220 & & & \\
\hline \multirow[t]{2}{*}{$\mathrm{C}_{4} \mathrm{H}_{4}$} & methylenecyclopropene & {$[\mathrm{T}-(\mathrm{T})]$} & -0.263 & -0.284 & -0.257 & & \\
\hline & & {$[(\mathrm{Q})-\mathrm{T}]$} & 0.265 & 0.277 & & & \\
\hline \multirow[t]{2}{*}{$\mathrm{C}_{4} \mathrm{H}_{4}$} & tetrahedrane & {$[\mathrm{T}-(\mathrm{T})]$} & -0.460 & -0.487 & -0.452 & -0.464 & \\
\hline & & {$[(\mathrm{Q})-\mathrm{T}]$} & 0.436 & 0.487 & 0.463 & & \\
\hline \multirow[t]{2}{*}{$\mathrm{C}_{4} \mathrm{H}_{6}$} & 1,2-butadiene & {$[\mathrm{T}-(\mathrm{T})]$} & -0.083 & -0.102 & -0.091 & & \\
\hline & & {$[(\mathrm{Q})-\mathrm{T}]$} & 0.114 & 0.136 & & & \\
\hline \multirow[t]{2}{*}{$\mathrm{C}_{4} \mathrm{H}_{6}$} & 1,3-butadiene & {$[\mathrm{T}-(\mathrm{T})]$} & -0.067 & -0.080 & -0.073 & & \\
\hline & & {$[(\mathrm{Q})-\mathrm{T}]$} & 0.167 & 0.198 & & & \\
\hline \multirow[t]{2}{*}{$\mathrm{C}_{4} \mathrm{H}_{6}$} & 2-butyne & {$[\mathrm{T}-(\mathrm{T})]$} & -0.070 & -0.079 & -0.074 & & \\
\hline & & {$[(\mathrm{Q})-\mathrm{T}]$} & 0.079 & 0.089 & & & \\
\hline \multirow[t]{2}{*}{$\mathrm{C}_{4} \mathrm{H}_{6}$} & bicyclo[1.1.0]butane & {$[\mathrm{T}-(\mathrm{T})]$} & -0.316 & -0.317 & -0.304 & & \\
\hline & & {$[(\mathrm{Q})-\mathrm{T}]$} & 0.316 & 0.332 & & & \\
\hline \multirow[t]{2}{*}{$\mathrm{C}_{4} \mathrm{H}_{6}$} & cyclobutene & {$[\mathrm{T}-(\mathrm{T})]$} & -0.225 & -0.238 & -0.228 & & \\
\hline & & {$[(\mathrm{Q})-\mathrm{T}]$} & 0.230 & 0.238 & & & \\
\hline \multirow[t]{2}{*}{$\mathrm{C}_{4} \mathrm{H}_{6}$} & methylenecyclopropane & {$[\mathrm{T}-(\mathrm{T})]$} & -0.168 & -0.159 & -0.147 & & \\
\hline & & {$[(\mathrm{Q})-\mathrm{T}]$} & 0.186 & 0.187 & & & \\
\hline \multirow[t]{2}{*}{$\mathrm{C}_{4} \mathrm{H}_{8}$} & cyclobutane & {$[\mathrm{T}-(\mathrm{T})]$} & -0.141 & -0.141 & -0.138 & & \\
\hline & & {$[(\mathrm{Q})-\mathrm{T}]$} & 0.122 & 0.130 & & & \\
\hline \multirow[t]{2}{*}{$\mathrm{C}_{4} \mathrm{H}_{8}$} & isobutene & {$[\mathrm{T}-(\mathrm{T})]$} & -0.102 & -0.110 & -0.108 & & \\
\hline & & {$[(\mathrm{Q})-\mathrm{T}]$} & 0.095 & 0.112 & & & \\
\hline \multirow[t]{2}{*}{$\mathrm{C}_{4} \mathrm{H}_{10}$} & isobutane & {$[\mathrm{T}-(\mathrm{T})]$} & -0.072 & & & & \\
\hline & & {$[(\mathrm{Q})-\mathrm{T}]$} & 0.052 & & & & \\
\hline \multirow[t]{2}{*}{$\mathrm{C}_{4} \mathrm{H}_{10}$} & $n$-butane & {$[\mathrm{T}-(\mathrm{T})]$} & -0.053 & -0.061 & -0.061 & & \\
\hline & & {$[(\mathrm{Q})-\mathrm{T}]$} & 0.039 & 0.053 & & & \\
\hline
\end{tabular}


Table S13, contd.

\begin{tabular}{|c|c|c|c|c|c|c|c|}
\hline & Molecule & Term & $\left(2,3^{t r}\right)$ & $\overline{3^{\prime}}$ & $\overline{3}$ & $\overline{\overline{4^{\prime}}}$ & $\overline{4}$ \\
\hline \multirow[t]{2}{*}{$\mathrm{C}_{5} \mathrm{H}_{4}$} & 1,4-pentadiyne & {$[\mathrm{T}-(\mathrm{T})]$} & -0.149 & -0.173 & & & \\
\hline & & {$[(\mathrm{Q})-\mathrm{T}]$} & 0.165 & 0.177 & & & \\
\hline \multirow[t]{2}{*}{$\mathrm{C}_{5} \mathrm{H}_{4}$} & pentatetraene & {$[\mathrm{T}-(\mathrm{T})]$} & -0.170 & -0.237 & -0.199 & & \\
\hline & & {$[(\mathrm{Q})-\mathrm{T}]$} & 0.683 & 0.777 & & & \\
\hline \multirow[t]{2}{*}{$\mathrm{C}_{5} \mathrm{H}_{4}$} & spiropentadiene & {$[\mathrm{T}-(\mathrm{T})]$} & -0.602 & -0.620 & -0.575 & & \\
\hline & & {$[(\mathrm{Q})-\mathrm{T}]$} & 0.621 & 0.639 & & & \\
\hline \multirow[t]{2}{*}{$\mathrm{C}_{5} \mathrm{H}_{6}$} & [1.1.1]propellane & {$[\mathrm{T}-(\mathrm{T})]$} & -0.804 & -0.828 & -0.798 & & \\
\hline & & {$[(\mathrm{Q})-\mathrm{T}]$} & 0.935 & 0.967 & & & \\
\hline \multirow[t]{2}{*}{$\mathrm{C}_{5} \mathrm{H}_{6}$} & 1,3-cyclopentadiene & {$[\mathrm{T}-(\mathrm{T})]$} & -0.369 & -0.405 & -0.389 & & \\
\hline & & {$[(\mathrm{Q})-\mathrm{T}]$} & 0.401 & 0.437 & & & \\
\hline \multirow[t]{2}{*}{$\mathrm{C}_{5} \mathrm{H}_{6}$} & 3-methylenecyclobutene & {$[\mathrm{T}-(\mathrm{T})]$} & -0.319 & & & & \\
\hline & & {$[(\mathrm{Q})-\mathrm{T}]$} & 0.384 & & & & \\
\hline \multirow[t]{2}{*}{$\mathrm{C}_{5} \mathrm{H}_{6}$} & bicyclo[2.1.0]pent-2-ene & {$[\mathrm{T}-(\mathrm{T})]$} & -0.491 & & & & \\
\hline & & {$[(\mathrm{Q})-\mathrm{T}]$} & 0.518 & & & & \\
\hline \multirow[t]{2}{*}{$\mathrm{C}_{5} \mathrm{H}_{6}$} & cyclopropylacetylene & {$[\mathrm{T}-(\mathrm{T})]$} & -0.246 & & & & \\
\hline & & {$[(\mathrm{Q})-\mathrm{T}]$} & 0.243 & & & & \\
\hline \multirow[t]{2}{*}{$\mathrm{C}_{5} \mathrm{H}_{8}$} & 1,4-pentadiene & {$[\mathrm{T}-(\mathrm{T})]$} & -0.155 & & & & \\
\hline & & {$[(\mathrm{Q})-\mathrm{T}]$} & 0.158 & & & & \\
\hline \multirow[t]{2}{*}{$\mathrm{C}_{5} \mathrm{H}_{8}$} & isoprene & {$[\mathrm{T}-(\mathrm{T})]$} & -0.159 & & & & \\
\hline & & {$[(\mathrm{Q})-\mathrm{T}]$} & 0.239 & & & & \\
\hline \multirow[t]{2}{*}{$\mathrm{C}_{5} \mathrm{H}_{8}$} & spiropentane & {$[\mathrm{T}-(\mathrm{T})]$} & -0.302 & -0.635 & -0.272 & & \\
\hline & & {$[(\mathrm{Q})-\mathrm{T}]$} & 0.258 & 0.834 & & & \\
\hline \multirow[t]{2}{*}{$\mathrm{C}_{5} \mathrm{H}_{10}$} & cyclopentane & {$[\mathrm{T}-(\mathrm{T})]$} & -0.175 & & & & \\
\hline & & {$[(\mathrm{Q})-\mathrm{T}]$} & 0.120 & & & & \\
\hline \multirow[t]{2}{*}{$\mathrm{C}_{5} \mathrm{H}_{12}$} & neopentane & {$[\mathrm{T}-(\mathrm{T})]$} & -0.155 & & & & \\
\hline & & {$[(\mathrm{Q})-\mathrm{T}]$} & 0.100 & & & & \\
\hline
\end{tabular}


Table S13, contd.

\begin{tabular}{|c|c|c|c|c|c|c|c|}
\hline & Molecule & Term & $\left(2,3^{t r}\right)$ & $\overline{3^{\prime}}$ & $\overline{3}$ & $\overline{4^{\prime}}$ & $\overline{4}$ \\
\hline \multirow[t]{2}{*}{$\mathrm{C}_{5} \mathrm{H}_{12}$} & $n$-pentane & {$[\mathrm{T}-(\mathrm{T})]$} & -0.084 & & & & \\
\hline & & {$[(\mathrm{Q})-\mathrm{T}]$} & 0.061 & & & & \\
\hline \multirow[t]{2}{*}{$\mathrm{C}_{6} \mathrm{H}_{2}$} & 1,3,5-hexatriyne & {$[\mathrm{T}-(\mathrm{T})]$} & -0.477 & -0.580 & -0.543 & & \\
\hline & & {$[(\mathrm{Q})-\mathrm{T}]$} & 0.974 & 1.032 & & & \\
\hline \multirow[t]{2}{*}{$\mathrm{C}_{6} \mathrm{H}_{4}$} & butalene & {$[\mathrm{T}-(\mathrm{T})]$} & -1.316 & -1.486 & & & \\
\hline & & {$[(\mathrm{Q})-\mathrm{T}]$} & 1.528 & 1.638 & & & \\
\hline \multirow[t]{2}{*}{$\mathrm{C}_{6} \mathrm{H}_{4}$} & cis-3-hexene-1,5-diyne & {$[\mathrm{T}-(\mathrm{T})]$} & -0.286 & & & & \\
\hline & & {$[(\mathrm{Q})-\mathrm{T}]$} & 0.495 & & & & \\
\hline \multirow[t]{2}{*}{$\mathrm{C}_{6} \mathrm{H}_{4}$} & hexapentaene & {$[\mathrm{T}-(\mathrm{T})]$} & -0.214 & -0.313 & -0.260 & & \\
\hline & & {$[(\mathrm{Q})-\mathrm{T}]$} & 1.191 & 1.339 & & & \\
\hline \multirow[t]{2}{*}{$\mathrm{C}_{6} \mathrm{H}_{4}$} & ortho-benzyne & {$[\mathrm{T}-(\mathrm{T})]$} & -1.058 & -1.208 & -1.175 & & \\
\hline & & {$[(\mathrm{Q})-\mathrm{T}]$} & 1.427 & 1.526 & & & \\
\hline \multirow[t]{2}{*}{$\mathrm{C}_{6} \mathrm{H}_{4}$} & trans-3-hexene-1,5-diyne & {$[\mathrm{T}-(\mathrm{T})]$} & -0.259 & -0.307 & -0.280 & & \\
\hline & & {$[(\mathrm{Q})-\mathrm{T}]$} & 0.476 & 0.529 & & & \\
\hline \multirow[t]{2}{*}{$\mathrm{C}_{6} \mathrm{H}_{4}$} & triafulvalene & {$[\mathrm{T}-(\mathrm{T})]$} & -0.675 & -0.739 & -0.675 & & \\
\hline & & {$[(\mathrm{Q})-\mathrm{T}]$} & 0.737 & 0.767 & & & \\
\hline \multirow[t]{2}{*}{$\mathrm{C}_{6} \mathrm{H}_{6}$} & 2,4-hexadiyne & {$[\mathrm{T}-(\mathrm{T})]$} & -0.305 & -0.364 & -0.344 & & \\
\hline & & {$[(\mathrm{Q})-\mathrm{T}]$} & 0.509 & 0.548 & & & \\
\hline \multirow[t]{2}{*}{$\mathrm{C}_{6} \mathrm{H}_{6}$} & 3,3'-bicyclopropenyl & {$[\mathrm{T}-(\mathrm{T})]$} & -0.630 & -0.654 & & & \\
\hline & & {$[(\mathrm{Q})-\mathrm{T}]$} & 0.615 & 0.638 & & & \\
\hline \multirow[t]{2}{*}{$\mathrm{C}_{6} \mathrm{H}_{6}$} & 3,4-dimethylenecyclobutene & {$[\mathrm{T}-(\mathrm{T})]$} & -0.424 & -0.483 & & & \\
\hline & & {$[(\mathrm{Q})-\mathrm{T}]$} & 0.581 & 0.655 & & & \\
\hline \multirow[t]{2}{*}{$\mathrm{C}_{6} \mathrm{H}_{6}$} & [3]radialene & {$[\mathrm{T}-(\mathrm{T})]$} & -0.313 & -0.343 & & & \\
\hline & & {$[(\mathrm{Q})-\mathrm{T}]$} & 0.724 & 0.782 & & & \\
\hline \multirow[t]{2}{*}{$\mathrm{C}_{6} \mathrm{H}_{6}$} & benzene & {$[\mathrm{T}-(\mathrm{T})]$} & -0.636 & -0.743 & -0.714 & & \\
\hline & & {$[(\mathrm{Q})-\mathrm{T}]$} & 0.441 & 0.546 & & & \\
\hline
\end{tabular}


Table S13, contd.

\begin{tabular}{|c|c|c|c|c|c|c|c|}
\hline & Molecule & Term & $\left(2,3^{t r}\right)$ & $\overline{\overline{3^{\prime}}}$ & $\overline{3}$ & $\overline{\overline{4^{\prime}}}$ & $\overline{4}$ \\
\hline \multirow[t]{2}{*}{$\mathrm{C}_{6} \mathrm{H}_{6}$} & benzvalene & {$[\mathrm{T}-(\mathrm{T})]$} & -0.730 & -0.773 & & & \\
\hline & & {$[(\mathrm{Q})-\mathrm{T}]$} & 0.665 & 0.718 & & & \\
\hline \multirow[t]{2}{*}{$\mathrm{C}_{6} \mathrm{H}_{6}$} & Dewar benzene & {$[\mathrm{T}-(\mathrm{T})]$} & -0.687 & -0.735 & & & \\
\hline & & {$[(\mathrm{Q})-\mathrm{T}]$} & 0.675 & 0.704 & & & \\
\hline \multirow[t]{2}{*}{$\mathrm{C}_{6} \mathrm{H}_{6}$} & fulvene & {$[\mathrm{T}-(\mathrm{T})]$} & -0.504 & -0.584 & -0.552 & & \\
\hline & & {$[(\mathrm{Q})-\mathrm{T}]$} & 0.726 & 0.785 & & & \\
\hline \multirow[t]{2}{*}{$\mathrm{C}_{6} \mathrm{H}_{6}$} & prismane & {$[\mathrm{T}-(\mathrm{T})]$} & -0.660 & -0.716 & -0.669 & & \\
\hline & & {$[(\mathrm{Q})-\mathrm{T}]$} & 0.645 & 0.700 & & & \\
\hline \multirow[t]{2}{*}{$\mathrm{C}_{6} \mathrm{H}_{8}$} & 1,3-cyclohexadiene & {$[\mathrm{T}-(\mathrm{T})]$} & -0.340 & & & & \\
\hline & & {$[(\mathrm{Q})-\mathrm{T}]$} & 0.391 & & & & \\
\hline \multirow[t]{2}{*}{$\mathrm{C}_{6} \mathrm{H}_{8}$} & 1,4-cyclohexadiene & {$[\mathrm{T}-(\mathrm{T})]$} & -0.295 & -0.315 & -0.302 & & \\
\hline & & {$[(\mathrm{Q})-\mathrm{T}]$} & 0.265 & 0.294 & & & \\
\hline \multirow[t]{2}{*}{$\mathrm{C}_{6} \mathrm{H}_{8}$} & trans-1,3,5-hexatriene & {$[\mathrm{T}-(\mathrm{T})]$} & -0.154 & & & & \\
\hline & & {$[(\mathrm{Q})-\mathrm{T}]$} & 0.411 & & & & \\
\hline \multirow[t]{2}{*}{$\mathrm{C}_{6} \mathrm{H}_{10}$} & cyclohexene & {$[\mathrm{T}-(\mathrm{T})]$} & -0.254 & & & & \\
\hline & & {$[(\mathrm{Q})-\mathrm{T}]$} & 0.200 & & & & \\
\hline \multirow[t]{2}{*}{$\mathrm{C}_{6} \mathrm{H}_{12}$} & cyclohexane & {$[\mathrm{T}-(\mathrm{T})]$} & -0.191 & & & & \\
\hline & & {$[(\mathrm{Q})-\mathrm{T}]$} & 0.127 & & & & \\
\hline \multirow[t]{2}{*}{$\mathrm{C}_{7} \mathrm{H}_{4}$} & 3,3-diethynylcyclopropene & {$[\mathrm{T}-(\mathrm{T})]$} & -0.648 & & & & \\
\hline & & {$[(\mathrm{Q})-\mathrm{T}]$} & 0.654 & & & & \\
\hline \multirow[t]{2}{*}{$\mathrm{C}_{7} \mathrm{H}_{4}$} & heptahexaene & {$[\mathrm{T}-(\mathrm{T})]$} & -0.390 & & & & \\
\hline & & {$[(\mathrm{Q})-\mathrm{T}]$} & 1.725 & & & & \\
\hline \multirow[t]{2}{*}{$\mathrm{C}_{7} \mathrm{H}_{6}$} & 1,1-diethynylcyclopropane & {$[\mathrm{T}-(\mathrm{T})]$} & -0.484 & & & & \\
\hline & & {$[(\mathrm{Q})-\mathrm{T}]$} & 0.475 & & & & \\
\hline \multirow[t]{2}{*}{$\mathrm{C}_{7} \mathrm{H}_{6}$} & 1,3,5-norcaratriene & {$[\mathrm{T}-(\mathrm{T})]$} & -1.031 & & & & \\
\hline & & {$[(\mathrm{Q})-\mathrm{T}]$} & 0.833 & & & & \\
\hline
\end{tabular}


Table S13, contd.

\begin{tabular}{|c|c|c|c|c|c|c|c|}
\hline & Molecule & Term & $\left(2,3^{t r}\right)$ & $\overline{\overline{3^{\prime}}}$ & $\overline{3}$ & $\overline{\overline{4^{\prime}}}$ & $\overline{4}$ \\
\hline \multirow[t]{2}{*}{$\mathrm{C}_{7} \mathrm{H}_{6}$} & fulvenallene & {$[\mathrm{T}-(\mathrm{T})]$} & -0.656 & & & & \\
\hline & & {$[(\mathrm{Q})-\mathrm{T}]$} & 0.860 & & & & \\
\hline \multirow[t]{2}{*}{$\mathrm{C}_{7} \mathrm{H}_{6}$} & spiro[2.4]hepta-1,4,6-triene & {$[\mathrm{T}-(\mathrm{T})]$} & -0.885 & & & & \\
\hline & & {$[(\mathrm{Q})-\mathrm{T}]$} & 0.906 & & & & \\
\hline \multirow[t]{2}{*}{$\mathrm{C}_{7} \mathrm{H}_{8}$} & 1,3,5-cycloheptatriene & {$[\mathrm{T}-(\mathrm{T})]$} & -0.676 & & & & \\
\hline & & {$[(\mathrm{Q})-\mathrm{T}]$} & 0.771 & & & & \\
\hline \multirow[t]{2}{*}{$\mathrm{C}_{7} \mathrm{H}_{8}$} & 2,5 -norbornadiene & {$[\mathrm{T}-(\mathrm{T})]$} & -0.794 & & & & \\
\hline & & {$[(\mathrm{Q})-\mathrm{T}]$} & 0.718 & & & & \\
\hline \multirow[t]{2}{*}{$\mathrm{C}_{7} \mathrm{H}_{8}$} & norcaradiene & {$[\mathrm{T}-(\mathrm{T})]$} & -0.679 & & & & \\
\hline & & {$[(\mathrm{Q})-\mathrm{T}]$} & 0.727 & & & & \\
\hline \multirow[t]{2}{*}{$\mathrm{C}_{7} \mathrm{H}_{8}$} & quadricyclane & {$[\mathrm{T}-(\mathrm{T})]$} & -0.751 & & & & \\
\hline & & {$[(\mathrm{Q})-\mathrm{T}]$} & 0.599 & & & & \\
\hline \multirow[t]{2}{*}{$\mathrm{C}_{7} \mathrm{H}_{8}$} & spiro[2.4]hepta-4,6-diene & {$[\mathrm{T}-(\mathrm{T})]$} & -0.729 & & & & \\
\hline & & {$[(\mathrm{Q})-\mathrm{T}]$} & 0.716 & & & & \\
\hline \multirow[t]{2}{*}{$\mathrm{C}_{8} \mathrm{H}_{2}$} & 1,3,5,7-octatetrayne & {$[\mathrm{T}-(\mathrm{T})]$} & -0.832 & & & & \\
\hline & & {$[(\mathrm{Q})-\mathrm{T}]$} & 1.691 & & & & \\
\hline \multirow[t]{2}{*}{$\mathrm{C}_{8} \mathrm{H}_{4}$} & 1,2,3,5,6,7-cyclooctahexaene & {$[\mathrm{T}-(\mathrm{T})]$} & -0.652 & & & & \\
\hline & & {$[(\mathrm{Q})-\mathrm{T}]$} & 2.229 & & & & \\
\hline \multirow[t]{2}{*}{$\mathrm{C}_{8} \mathrm{H}_{4}$} & 1,5-cyclooctadiene-3,7-diyne & {$[\mathrm{T}-(\mathrm{T})]$} & -0.895 & & & & \\
\hline & & {$[(\mathrm{Q})-\mathrm{T}]$} & 1.702 & & & & \\
\hline \multirow[t]{2}{*}{$\mathrm{C}_{8} \mathrm{H}_{4}$} & octaheptaene & {$[\mathrm{T}-(\mathrm{T})]$} & -0.430 & & & & \\
\hline & & {$[(\mathrm{Q})-\mathrm{T}]$} & 2.385 & & & & \\
\hline \multirow[t]{2}{*}{$\mathrm{C}_{8} \mathrm{H}_{6}$} & benzocyclobutadiene & {$[\mathrm{T}-(\mathrm{T})]$} & -1.017 & & & & \\
\hline & & {$[(\mathrm{Q})-\mathrm{T}]$} & 1.119 & & & & \\
\hline \multirow[t]{2}{*}{$\mathrm{C}_{8} \mathrm{H}_{6}$} & bis(cyclopropa)benzene & {$[\mathrm{T}-(\mathrm{T})]$} & -1.467 & & & & \\
\hline & & {$[(\mathrm{Q})-\mathrm{T}]$} & 1.246 & & & & \\
\hline
\end{tabular}


Table S13, contd.

\begin{tabular}{|c|c|c|c|c|c|c|c|}
\hline & Molecule & Term & $\left(2,3^{t r}\right)$ & $\overline{3^{\prime}}$ & $\overline{3}$ & $\overline{4^{\prime}}$ & $\overline{4}$ \\
\hline \multirow[t]{2}{*}{$\mathrm{C}_{8} \mathrm{H}_{6}$} & pentalene & {$[\mathrm{T}-(\mathrm{T})]$} & -0.967 & & & & \\
\hline & & {$[(\mathrm{Q})-\mathrm{T}]$} & 1.501 & & & & \\
\hline \multirow[t]{2}{*}{$\mathrm{C}_{8} \mathrm{H}_{8}$} & 1,3,5,7-cyclooctatetraene & {$[\mathrm{T}-(\mathrm{T})]$} & -0.646 & & & & \\
\hline & & {$[(\mathrm{Q})-\mathrm{T}]$} & 0.752 & & & & \\
\hline \multirow[t]{2}{*}{$\mathrm{C}_{8} \mathrm{H}_{8}$} & benzocyclobutene & {$[\mathrm{T}-(\mathrm{T})]$} & -1.000 & & & & \\
\hline & & {$[(\mathrm{Q})-\mathrm{T}]$} & 0.735 & & & & \\
\hline \multirow[t]{2}{*}{$\mathrm{C}_{8} \mathrm{H}_{8}$} & cubane & {$[\mathrm{T}-(\mathrm{T})]$} & -0.811 & & & & \\
\hline & & {$[(\mathrm{Q})-\mathrm{T}]$} & 0.810 & & & & \\
\hline \multirow[t]{2}{*}{$\mathrm{C}_{8} \mathrm{H}_{8}$} & para-xylylene & {$[\mathrm{T}-(\mathrm{T})]$} & -0.423 & & & & \\
\hline & & {$[(\mathrm{Q})-\mathrm{T}]$} & 1.075 & & & & \\
\hline \multirow[t]{2}{*}{$\mathrm{C}_{10} \mathrm{H}_{4}$} & tetraethynylethene & {$[\mathrm{T}-(\mathrm{T})]$} & -0.864 & & & & \\
\hline & & {$[(\mathrm{Q})-\mathrm{T}]$} & 1.313 & & & & \\
\hline \multirow[t]{2}{*}{$\mathrm{C}_{10} \mathrm{H}_{8}$} & naphthalene & {$[\mathrm{T}-(\mathrm{T})]$} & -1.450 & & & & \\
\hline & & {$[(\mathrm{Q})-\mathrm{T}]$} & 1.215 & & & & \\
\hline \multirow[t]{2}{*}{$\mathrm{C}_{10} \mathrm{H}_{8}$} & pentafulvalene & {$[\mathrm{T}-(\mathrm{T})]$} & -1.192 & & & & \\
\hline & & {$[(\mathrm{Q})-\mathrm{T}]$} & 1.705 & & & & \\
\hline
\end{tabular}

${ }^{a}$ Basis sets are defined by their cardinal number as given in the header row (notation as in Sec 3). See text for further details and for definitions of terms. Note that bond separation energy contributions are zero by definition for all BSR prototypes (methane, ethane, ethylene, acetylene) and so not listed here. 
Table S14: Higher order electron correlation contributions to atomization energies (kcal $/ \mathrm{mol}):$ Summary ${ }^{a}$

\begin{tabular}{|c|c|c|c|c|c|c|c|c|c|}
\hline & \multirow[t]{2}{*}{ Molecule } & \multirow[t]{2}{*}{$\mathrm{MID}^{b}$} & \multicolumn{3}{|c|}{$[(\mathrm{Q})-(\mathrm{T})] \mathrm{BSE}^{c}$} & \multicolumn{3}{|c|}{ Thermoneutral BSR evaluation } & \multirow[t]{2}{*}{ Best est. } \\
\hline & & & $\left(2,3^{t r}\right)$ & $\overline{3^{\prime}}$ & $\overline{4^{\prime}}$ & Value & Unc. & ATOMIC(hc) & \\
\hline $\mathrm{CH}_{4}$ & methane & & 0.00 & 0.00 & 0.00 & $0.00 \pm 0.02$ & 0.00 & $0.00 \pm 0.02$ & $0.00 \pm 0.02$ \\
\hline $\mathrm{C}_{2} \mathrm{H}_{2}$ & acetylene & & 0.00 & 0.00 & 0.00 & $0.10 \pm 0.04$ & 0.03 & $0.10 \pm 0.05$ & $0.10 \pm 0.05$ \\
\hline $\mathrm{C}_{2} \mathrm{H}_{4}$ & ethylene & & 0.00 & 0.00 & 0.00 & $0.02 \pm 0.04$ & 0.03 & $0.02 \pm 0.05$ & $0.02 \pm 0.05$ \\
\hline $\mathrm{C}_{2} \mathrm{H}_{6}$ & ethane & & 0.00 & 0.00 & 0.00 & $-0.10 \pm 0.04$ & 0.03 & $-0.10 \pm 0.05$ & $-0.10 \pm 0.05$ \\
\hline $\mathrm{C}_{3} \mathrm{H}_{4}$ & allene & 46 & 0.04 & 0.04 & 0.04 & $0.04 \pm 0.06$ & 0.26 & $0.04 \pm 0.27$ & $0.08 \pm 0.08$ \\
\hline $\mathrm{C}_{3} \mathrm{H}_{4}$ & cyclopropene & 42 & 0.02 & 0.02 & 0.04 & $-0.17 \pm 0.07$ & 0.09 & $-0.17 \pm 0.11$ & $-0.15 \pm 0.11$ \\
\hline $\mathrm{C}_{3} \mathrm{H}_{4}$ & propyne & 33 & 0.00 & 0.01 & 0.00 & $0.00 \pm 0.07$ & 0.06 & $0.00 \pm 0.09$ & $0.01 \pm 0.09$ \\
\hline $\mathrm{C}_{3} \mathrm{H}_{6}$ & cyclopropane & 30 & 0.00 & 0.01 & 0.02 & $-0.29 \pm 0.07$ & 0.09 & $-0.29 \pm 0.12$ & $-0.29 \pm 0.12$ \\
\hline $\mathrm{C}_{3} \mathrm{H}_{6}$ & propene & 35 & 0.01 & 0.01 & & $-0.07 \pm 0.06$ & 0.06 & $-0.07 \pm 0.08$ & $-0.07 \pm 0.08$ \\
\hline $\mathrm{C}_{3} \mathrm{H}_{8}$ & propane & 26 & 0.00 & 0.00 & & $-0.19 \pm 0.06$ & 0.06 & $-0.19 \pm 0.09$ & $-0.20 \pm 0.09$ \\
\hline $\mathrm{C}_{4} \mathrm{H}_{2}$ & 1,3-butadiyne & 61 & 0.21 & 0.19 & 0.19 & $0.10 \pm 0.09$ & 0.49 & $0.10 \pm 0.50$ & $0.31 \pm 0.13$ \\
\hline $\mathrm{C}_{4} \mathrm{H}_{4}$ & bicyclo[1.1.0]but-1(3)-ene & 77 & 0.68 & 0.63 & & $-0.36 \pm 0.10$ & 0.15 & $-0.36 \pm 0.18$ & $0.32 \pm 0.18$ \\
\hline $\mathrm{C}_{4} \mathrm{H}_{4}$ & butatriene & 67 & 0.30 & 0.33 & 0.33 & $0.07 \pm 0.08$ & 0.39 & $0.07 \pm 0.40$ & $0.36 \pm 0.12$ \\
\hline $\mathrm{C}_{4} \mathrm{H}_{4}$ & cyclobutadiene & 71 & 0.48 & 0.50 & 0.52 & $-0.15 \pm 0.09$ & 0.32 & $-0.15 \pm 0.33$ & $0.34 \pm 0.15$ \\
\hline $\mathrm{C}_{4} \mathrm{H}_{4}$ & ethynylethene & 52 & 0.09 & 0.09 & & $0.02 \pm 0.09$ & 0.39 & $0.02 \pm 0.40$ & $0.11 \pm 0.13$ \\
\hline $\mathrm{C}_{4} \mathrm{H}_{4}$ & methylenecyclopropene & 31 & 0.00 & -0.01 & & $-0.15 \pm 0.09$ & 0.32 & $-0.15 \pm 0.33$ & $-0.15 \pm 0.15$ \\
\hline $\mathrm{C}_{4} \mathrm{H}_{4}$ & tetrahedrane & 15 & -0.02 & 0.00 & & $-0.58 \pm 0.11$ & 0.18 & $-0.58 \pm 0.21$ & $-0.60 \pm 0.21$ \\
\hline $\mathrm{C}_{4} \mathrm{H}_{6}$ & 1,2-butadiene & 44 & 0.03 & 0.03 & & $-0.05 \pm 0.08$ & 0.29 & $-0.05 \pm 0.30$ & $-0.02 \pm 0.12$ \\
\hline $\mathrm{C}_{4} \mathrm{H}_{6}$ & 1,3-butadiene & 54 & 0.10 & 0.12 & & $-0.05 \pm 0.08$ & 0.29 & $-0.05 \pm 0.30$ & $0.05 \pm 0.12$ \\
\hline $\mathrm{C}_{4} \mathrm{H}_{6}$ & 2-butyne & 37 & 0.01 & 0.01 & & $-0.09 \pm 0.09$ & 0.09 & $-0.09 \pm 0.13$ & $-0.08 \pm 0.13$ \\
\hline $\mathrm{C}_{4} \mathrm{H}_{6}$ & bicyclo[1.1.0]butane & 28 & 0.00 & 0.01 & & $-0.48 \pm 0.10$ & 0.15 & $-0.48 \pm 0.18$ & $-0.48 \pm 0.18$ \\
\hline $\mathrm{C}_{4} \mathrm{H}_{6}$ & cyclobutene & 34 & 0.01 & 0.00 & & $-0.27 \pm 0.09$ & 0.12 & $-0.27 \pm 0.15$ & $-0.26 \pm 0.15$ \\
\hline $\mathrm{C}_{4} \mathrm{H}_{6}$ & methylenecyclopropane & 39 & 0.02 & 0.03 & & $-0.27 \pm 0.09$ & 0.12 & $-0.27 \pm 0.15$ & $-0.25 \pm 0.15$ \\
\hline $\mathrm{C}_{4} \mathrm{H}_{8}$ & cyclobutane & 18 & -0.02 & -0.01 & & $-0.38 \pm 0.10$ & 0.12 & $-0.38 \pm 0.15$ & $-0.40 \pm 0.15$ \\
\hline $\mathrm{C}_{4} \mathrm{H}_{8}$ & isobutene & 25 & -0.01 & 0.00 & & $-0.17 \pm 0.08$ & 0.09 & $-0.17 \pm 0.12$ & $-0.18 \pm 0.12$ \\
\hline $\mathrm{C}_{4} \mathrm{H}_{10}$ & isobutane & 17 & -0.02 & & & $-0.29 \pm 0.09$ & 0.09 & $-0.29 \pm 0.13$ & $-0.31 \pm 0.13$ \\
\hline $\mathrm{C}_{4} \mathrm{H}_{10}$ & $n$-butane & 21 & -0.01 & -0.01 & & $-0.29 \pm 0.09$ & 0.09 & $-0.29 \pm 0.13$ & $-0.30 \pm 0.13$ \\
\hline $\mathrm{C}_{5} \mathrm{H}_{4}$ & 1,4-pentadiyne & 38 & 0.02 & 0.00 & & $0.00 \pm 0.12$ & 0.12 & $0.00 \pm 0.17$ & $0.02 \pm 0.17$ \\
\hline $\mathrm{C}_{5} \mathrm{H}_{4}$ & pentatetraene & 73 & 0.51 & 0.54 & & $0.09 \pm 0.10$ & 0.52 & $0.09 \pm 0.53$ & $0.60 \pm 0.15$ \\
\hline $\mathrm{C}_{5} \mathrm{H}_{4}$ & spiropentadiene & 40 & 0.02 & 0.02 & & $-0.34 \pm 0.12$ & 0.18 & $-0.34 \pm 0.22$ & $-0.32 \pm 0.22$ \\
\hline $\mathrm{C}_{5} \mathrm{H}_{6}$ & [1.1.1]propellane & 57 & 0.13 & 0.14 & & $-0.67 \pm 0.14$ & 0.21 & $-0.67 \pm 0.25$ & $-0.54 \pm 0.25$ \\
\hline $\mathrm{C}_{5} \mathrm{H}_{6}$ & 1,3-cyclopentadiene & 45 & 0.03 & 0.03 & & $-0.24 \pm 0.11$ & 0.35 & $-0.24 \pm 0.37$ & $-0.21 \pm 0.19$ \\
\hline $\mathrm{C}_{5} \mathrm{H}_{6}$ & 3-methylenecyclobutene & 50 & 0.07 & & & $-0.24 \pm 0.11$ & 0.35 & $-0.24 \pm 0.37$ & $-0.18 \pm 0.19$ \\
\hline $\mathrm{C}_{5} \mathrm{H}_{6}$ & bicyclo[2.1.0]pent-2-ene & 43 & 0.03 & & & $-0.46 \pm 0.12$ & 0.18 & $-0.46 \pm 0.22$ & $-0.43 \pm 0.22$ \\
\hline $\mathrm{C}_{5} \mathrm{H}_{6}$ & cyclopropylacetylene & 27 & 0.00 & & & $-0.29 \pm 0.12$ & 0.15 & $-0.29 \pm 0.19$ & $-0.29 \pm 0.19$ \\
\hline $\mathrm{C}_{5} \mathrm{H}_{8}$ & 1,4-pentadiene & 32 & 0.00 & & & $-0.15 \pm 0.10$ & 0.12 & $-0.15 \pm 0.16$ & $-0.15 \pm 0.16$ \\
\hline $\mathrm{C}_{5} \mathrm{H}_{8}$ & isoprene & 51 & 0.08 & & & $-0.15 \pm 0.10$ & 0.32 & $-0.15 \pm 0.34$ & $-0.07 \pm 0.16$ \\
\hline
\end{tabular}


Table S14, contd.

\begin{tabular}{|c|c|c|c|c|c|c|c|c|c|}
\hline & \multirow[t]{2}{*}{ Molecule } & \multirow[t]{2}{*}{$\mathrm{MID}^{b}$} & \multicolumn{3}{|c|}{$[(\mathrm{Q})-(\mathrm{T})] \mathrm{BSE}^{c}$} & \multicolumn{3}{|c|}{ Thermoneutral BSR evaluation } & \multirow[t]{2}{*}{ Best est. } \\
\hline & & & $\left(2,3^{\text {tr }}\right)$ & $\overline{3^{\prime}}$ & $\overline{\overline{4^{\prime}}}$ & Value & Unc. & ATOMIC(hc) & \\
\hline $\mathrm{C}_{5} \mathrm{H}_{8}$ & spiropentane & 13 & -0.04 & 0.20 & & $-0.58 \pm 0.13$ & 0.18 & $-0.58 \pm 0.22$ & $-0.62 \pm 0.22$ \\
\hline $\mathrm{C}_{5} \mathrm{H}_{10}$ & cyclopentane & 11 & -0.05 & & & $-0.48 \pm 0.12$ & 0.15 & $-0.48 \pm 0.19$ & $-0.53 \pm 0.19$ \\
\hline $\mathrm{C}_{5} \mathrm{H}_{12}$ & neopentane & 10 & -0.05 & & & $-0.38 \pm 0.11$ & 0.12 & $-0.38 \pm 0.16$ & $-0.44 \pm 0.16$ \\
\hline $\mathrm{C}_{5} \mathrm{H}_{12}$ & $n$-pentane & 16 & -0.02 & & & $-0.38 \pm 0.11$ & 0.12 & $-0.38 \pm 0.16$ & $-0.41 \pm 0.16$ \\
\hline $\mathrm{C}_{6} \mathrm{H}_{2}$ & 1,3,5-hexatriyne & 72 & 0.50 & 0.45 & & $0.10 \pm 0.15$ & 0.75 & $0.10 \pm 0.76$ & $0.60 \pm 0.21$ \\
\hline $\mathrm{C}_{6} \mathrm{H}_{4}$ & butalene & 63 & 0.21 & 0.15 & & $-0.32 \pm 0.14$ & 0.51 & $-0.32 \pm 0.53$ & $-0.11 \pm 0.25$ \\
\hline $\mathrm{C}_{6} \mathrm{H}_{4}$ & cis-3-hexene-1,5-diyne & 62 & 0.21 & & & $0.03 \pm 0.14$ & 0.65 & $0.03 \pm 0.66$ & $0.24 \pm 0.20$ \\
\hline $\mathrm{C}_{6} \mathrm{H}_{4}$ & hexapentaene & 80 & 0.98 & 1.03 & & $0.11 \pm 0.12$ & 0.65 & $0.11 \pm 0.66$ & $1.09 \pm 0.19$ \\
\hline $\mathrm{C}_{6} \mathrm{H}_{4}$ & ortho-benzyne & 68 & 0.37 & 0.32 & & $-0.15 \pm 0.14$ & 0.58 & $-0.15 \pm 0.60$ & $0.22 \pm 0.23$ \\
\hline $\mathrm{C}_{6} \mathrm{H}_{4}$ & trans-3-hexene-1,5-diyne & 64 & 0.22 & 0.22 & & $0.03 \pm 0.14$ & 0.65 & $0.03 \pm 0.66$ & $0.24 \pm 0.20$ \\
\hline $\mathrm{C}_{6} \mathrm{H}_{4}$ & triafulvalene & 49 & 0.06 & 0.03 & & $-0.32 \pm 0.14$ & 0.51 & $-0.32 \pm 0.53$ & $-0.26 \pm 0.25$ \\
\hline $\mathrm{C}_{6} \mathrm{H}_{6}$ & 2,4-hexadiyne & 59 & 0.20 & 0.18 & & $-0.09 \pm 0.14$ & 0.55 & $-0.09 \pm 0.57$ & $0.11 \pm 0.21$ \\
\hline $\mathrm{C}_{6} \mathrm{H}_{6}$ & 3,3'-bicyclopropenyl & 19 & -0.01 & -0.02 & & $-0.44 \pm 0.14$ & 0.21 & $-0.44 \pm 0.25$ & $-0.45 \pm 0.25$ \\
\hline $\mathrm{C}_{6} \mathrm{H}_{6}$ & 3,4-dimethylenecyclobutene & 58 & 0.16 & 0.17 & & $-0.22 \pm 0.13$ & 0.48 & $-0.22 \pm 0.50$ & $-0.06 \pm 0.22$ \\
\hline $\mathrm{C}_{6} \mathrm{H}_{6}$ & [3]radialene & 69 & 0.41 & 0.44 & & $-0.22 \pm 0.13$ & 0.48 & $-0.22 \pm 0.50$ & $0.19 \pm 0.22$ \\
\hline $\mathrm{C}_{6} \mathrm{H}_{6}$ & benzene & 5 & -0.19 & -0.20 & & $-0.22 \pm 0.13$ & 0.48 & $-0.22 \pm 0.50$ & $-0.42 \pm 0.22$ \\
\hline $\mathrm{C}_{6} \mathrm{H}_{6}$ & benzvalene & 8 & -0.06 & -0.05 & & $-0.65 \pm 0.16$ & 0.24 & $-0.65 \pm 0.29$ & $-0.71 \pm 0.29$ \\
\hline $\mathrm{C}_{6} \mathrm{H}_{6}$ & Dewar benzene & 22 & -0.01 & -0.03 & & $-0.44 \pm 0.14$ & 0.21 & $-0.44 \pm 0.25$ & $-0.45 \pm 0.25$ \\
\hline $\mathrm{C}_{6} \mathrm{H}_{6}$ & fulvene & 65 & 0.22 & 0.20 & & $-0.22 \pm 0.13$ & 0.48 & $-0.22 \pm 0.50$ & $0.00 \pm 0.22$ \\
\hline $\mathrm{C}_{6} \mathrm{H}_{6}$ & prismane & 20 & -0.01 & -0.02 & & $-0.86 \pm 0.17$ & 0.27 & $-0.86 \pm 0.32$ & $-0.88 \pm 0.32$ \\
\hline $\mathrm{C}_{6} \mathrm{H}_{8}$ & 1,3-cyclohexadiene & 48 & 0.05 & & & $-0.34 \pm 0.14$ & 0.38 & $-0.34 \pm 0.40$ & $-0.29 \pm 0.23$ \\
\hline $\mathrm{C}_{6} \mathrm{H}_{8}$ & 1,4-cyclohexadiene & 14 & -0.03 & -0.02 & & $-0.34 \pm 0.14$ & 0.18 & $-0.34 \pm 0.23$ & $-0.37 \pm 0.23$ \\
\hline $\mathrm{C}_{6} \mathrm{H}_{8}$ & trans-1,3,5-hexatriene & 66 & 0.26 & & & $-0.13 \pm 0.12$ & 0.45 & $-0.13 \pm 0.47$ & $0.13 \pm 0.19$ \\
\hline $\mathrm{C}_{6} \mathrm{H}_{10}$ & cyclohexene & 12 & -0.05 & & & $-0.46 \pm 0.14$ & 0.18 & $-0.46 \pm 0.23$ & $-0.51 \pm 0.23$ \\
\hline $\mathrm{C}_{6} \mathrm{H}_{12}$ & cyclohexane & 9 & -0.06 & & & $-0.58 \pm 0.14$ & 0.18 & $-0.58 \pm 0.23$ & $-0.64 \pm 0.23$ \\
\hline $\mathrm{C}_{7} \mathrm{H}_{4}$ & 3,3-diethynylcyclopropene & 36 & 0.01 & & & $-0.17 \pm 0.17$ & 0.21 & $-0.17 \pm 0.27$ & $-0.16 \pm 0.27$ \\
\hline $\mathrm{C}_{7} \mathrm{H}_{4}$ & heptahexaene & 81 & 1.34 & & & $0.13 \pm 0.14$ & 0.78 & $0.13 \pm 0.79$ & $1.47 \pm 0.23$ \\
\hline $\mathrm{C}_{7} \mathrm{H}_{6}$ & 1,1-diethynylcyclopropane & 24 & -0.01 & & & $-0.28 \pm 0.17$ & 0.21 & $-0.28 \pm 0.27$ & $-0.29 \pm 0.27$ \\
\hline $\mathrm{C}_{7} \mathrm{H}_{6}$ & 1,3,5-norcaratriene & 4 & -0.20 & & & $-0.41 \pm 0.16$ & 0.54 & $-0.41 \pm 0.56$ & $-0.61 \pm 0.29$ \\
\hline $\mathrm{C}_{7} \mathrm{H}_{6}$ & fulvenallene & 60 & 0.21 & & & $-0.20 \pm 0.15$ & 0.61 & $-0.20 \pm 0.63$ & $0.01 \pm 0.26$ \\
\hline $\mathrm{C}_{7} \mathrm{H}_{6}$ & spiro[2.4]hepta-1,4,6-triene & 41 & 0.02 & & & $-0.41 \pm 0.16$ & 0.44 & $-0.41 \pm 0.47$ & $-0.39 \pm 0.29$ \\
\hline $\mathrm{C}_{7} \mathrm{H}_{8}$ & 1,3,5-cycloheptatriene & 53 & 0.10 & & & $-0.32 \pm 0.16$ & 0.51 & $-0.32 \pm 0.53$ & $-0.22 \pm 0.26$ \\
\hline $\mathrm{C}_{7} \mathrm{H}_{8}$ & 2,5 -norbornadiene & 7 & -0.08 & & & $-0.53 \pm 0.17$ & 0.24 & $-0.53 \pm 0.29$ & $-0.61 \pm 0.29$ \\
\hline $\mathrm{C}_{7} \mathrm{H}_{8}$ & norcaradiene & 47 & 0.05 & & & $-0.53 \pm 0.17$ & 0.44 & $-0.53 \pm 0.47$ & $-0.48 \pm 0.29$ \\
\hline $\mathrm{C}_{7} \mathrm{H}_{8}$ & quadricyclane & 6 & -0.15 & & & $-0.96 \pm 0.19$ & 0.30 & $-0.96 \pm 0.36$ & $-1.11 \pm 0.36$ \\
\hline $\mathrm{C}_{7} \mathrm{H}_{8}$ & spiro[2.4]hepta-4,6-diene & 23 & -0.01 & & & $-0.53 \pm 0.17$ & 0.44 & $-0.53 \pm 0.47$ & $-0.54 \pm 0.29$ \\
\hline $\mathrm{C}_{8} \mathrm{H}_{2}$ & 1,3,5,7-octatetrayne & 79 & 0.86 & & & $0.10 \pm 0.20$ & 1.01 & $0.10 \pm 1.03$ & $0.96 \pm 0.29$ \\
\hline $\mathrm{C}_{8} \mathrm{H}_{4}$ & $1,2,3,5,6,7$-cyclooctahexaene & 82 & 1.58 & & & $-0.06 \pm 0.17$ & 0.84 & $-0.06 \pm 0.86$ & $1.52 \pm 0.29$ \\
\hline
\end{tabular}


Table S14, contd.

\begin{tabular}{|c|c|c|c|c|c|c|c|c|c|}
\hline & \multirow[t]{2}{*}{ Molecule } & \multirow[t]{2}{*}{$\mathrm{MID}^{b}$} & \multicolumn{3}{|c|}{$[(\mathrm{Q})-(\mathrm{T})] \mathrm{BSE}^{c}$} & \multicolumn{3}{|c|}{ Thermoneutral BSR evaluation } & \multirow[t]{2}{*}{ Best est. } \\
\hline & & & $\overline{\left(2,3^{t r}\right)}$ & $\overline{3^{\prime}}$ & $\overline{\overline{4^{\prime}}}$ & Value & Unc. & ATOMIC(hc) & \\
\hline $\mathrm{C}_{8} \mathrm{H}_{4}$ & 1,5-cyclooctadiene-3,7-diyne & 78 & 0.81 & & & $-0.14 \pm 0.19$ & 0.84 & $-0.14 \pm 0.86$ & $0.66 \pm 0.31$ \\
\hline $\mathrm{C}_{8} \mathrm{H}_{4}$ & octaheptaene & 83 & 1.96 & & & $0.15 \pm 0.16$ & 0.91 & $0.15 \pm 0.92$ & $2.11 \pm 0.26$ \\
\hline $\mathrm{C}_{8} \mathrm{H}_{6}$ & benzocyclobutadiene & 55 & 0.10 & & & $-0.39 \pm 0.18$ & 0.67 & $-0.39 \pm 0.69$ & $-0.29 \pm 0.33$ \\
\hline $\mathrm{C}_{8} \mathrm{H}_{6}$ & bis(cyclopropa)benzene & 3 & -0.22 & & & $-0.61 \pm 0.20$ & 0.60 & $-0.61 \pm 0.63$ & $-0.83 \pm 0.36$ \\
\hline $\mathrm{C}_{8} \mathrm{H}_{6}$ & pentalene & 75 & 0.53 & & & $-0.39 \pm 0.18$ & 0.67 & $-0.39 \pm 0.69$ & $0.14 \pm 0.33$ \\
\hline $\mathrm{C}_{8} \mathrm{H}_{8}$ & 1,3,5,7-cyclooctatetraene & 56 & 0.11 & & & $-0.30 \pm 0.18$ & 0.64 & $-0.30 \pm 0.66$ & $-0.19 \pm 0.30$ \\
\hline $\mathrm{C}_{8} \mathrm{H}_{8}$ & benzocyclobutene & 1 & -0.26 & & & $-0.51 \pm 0.19$ & 0.57 & $-0.51 \pm 0.60$ & $-0.77 \pm 0.33$ \\
\hline $\mathrm{C}_{8} \mathrm{H}_{8}$ & cubane & 29 & 0.00 & & & $-1.15 \pm 0.22$ & 0.36 & $-1.15 \pm 0.42$ & $-1.15 \pm 0.42$ \\
\hline $\mathrm{C}_{8} \mathrm{H}_{8}$ & para-xylylene & 76 & 0.65 & & & $-0.30 \pm 0.18$ & 0.64 & $-0.30 \pm 0.66$ & $0.36 \pm 0.30$ \\
\hline $\mathrm{C}_{10} \mathrm{H}_{4}$ & tetraethynylethene & 70 & 0.45 & & & $0.03 \pm 0.24$ & 1.17 & $0.03 \pm 1.19$ & $0.48 \pm 0.36$ \\
\hline $\mathrm{C}_{10} \mathrm{H}_{8}$ & naphthalene & 2 & -0.23 & & & $-0.47 \pm 0.23$ & 0.83 & $-0.47 \pm 0.86$ & $-0.70 \pm 0.40$ \\
\hline $\mathrm{C}_{10} \mathrm{H}_{8}$ & pentafulvalene & 74 & 0.51 & & & $-0.47 \pm 0.23$ & 0.83 & $-0.47 \pm 0.86$ & $0.05 \pm 0.40$ \\
\hline
\end{tabular}

${ }^{a}$ The thermoneutral BSR evaluation simply sums over bond increments derived for BSR prototypes at the highest levels possible (Table 5). ATOMIC(hc) considers those values as well as the estimated uncertainties for the assumption of thermoneutrality (labeled "Unc.", compare Table 1). Note that the latter are obtained from a bond- and atom-increment system (Sec. 4.6) and so non-zero values are assigned even to BSR prototypes (ethane, ethylene, acetylene), although of course they should be zero in these cases. Actual deviations from thermoneutrality are computed as $\operatorname{CCSDT}(\mathrm{Q})-\mathrm{CCSD}(\mathrm{T})$ contributions to bond separation energies (BSEs); they are reported in the left three columns, and the value for $\left(2,3^{\text {tr }}\right)$, together with an estimated uncertainty of $0.03 \mathrm{kcal} / \mathrm{mol}$ per CC bond for even higher-order effects (see Sec. 4.6), is added to the thermoneutral evaluation to arrive at the best estimate (right column) for the correction toward FCI.

${ }^{b}$ Column "MID" reports the molecule index used in Figure 2. That figure also uses color coding to classify molecules. Most classifications should be self-explanatory, except "conjugated double, simple" (isolated 1,3-butadiene subunits, from left to right: spiro[2.4]-hepta-4,6-diene, spiro[2.4]-hepta-1,4,6-triene, 1,3-cyclopentadiene, norcaradiene, 1,3-cyclohexadiene, isoprene, 1,3-butadiene), and "conjugated double, other" (any conjugated double bond system not included in other classes, from left to right: 1,3,5norcaratriene, methylenecyclopropene, triafulvalene, 3-methylenecyclobutene, 1,3,5-cycloheptatriene, 1,3,5,7cyclooctatetraene, 3,4-dimethylenecyclobutene, butalene, fulvene, trans-1,3,5-hexatriene, [3]radialene, pentafulvalene, para-xylylene).

${ }^{c} X=\overline{\mathbf{3}}$ bond separation energies are available as well for all molecules for which $X=\overline{4^{\prime}}$ data are reported, additionally also for tetrahedrane. They agree with $X=\overline{\mathbf{3}^{\prime}}$ data to within $0.01 \mathrm{kcal} / \mathrm{mol}$ in all cases. 
Table S15: Electron correlation diagnostics ${ }^{a}$

\begin{tabular}{|c|c|c|c|c|c|c|c|c|}
\hline & Molecule & {$[(\mathrm{Q})-(\mathrm{T})] \mathrm{BSE}$} & $T_{1}\left(2_{s d}\right)$ & $T_{1}(4)$ & $\Delta T_{1}\left(2_{s d}\right)$ & $\Delta T_{1}(4)$ & $\% \mathrm{BSE}[(\mathrm{T})]$ & Note \\
\hline $\mathrm{C}_{2} \mathrm{H}_{2}$ & acetylene & 0.00 & 0.0125 & 0.0137 & 0.0000 & 0.0000 & 0.0 & \\
\hline $\mathrm{C}_{2} \mathrm{H}_{4}$ & ethylene & 0.00 & 0.0106 & 0.0111 & 0.0000 & 0.0000 & 0.0 & \\
\hline $\mathrm{C}_{2} \mathrm{H}_{6}$ & ethane & 0.00 & 0.0081 & 0.0081 & 0.0000 & 0.0000 & 0.0 & \\
\hline $\mathrm{C}_{3} \mathrm{H}_{4}$ & allene & 0.04 & 0.0124 & 0.0126 & 0.0018 & 0.0015 & 2.9 & \\
\hline $\mathrm{C}_{3} \mathrm{H}_{4}$ & cyclopropene & 0.02 & 0.0098 & 0.0102 & 0.0009 & 0.0011 & 6.7 & \\
\hline $\mathrm{C}_{3} \mathrm{H}_{4}$ & propyne & 0.00 & 0.0114 & 0.0119 & 0.0010 & 0.0010 & 1.0 & \\
\hline $\mathrm{C}_{3} \mathrm{H}_{6}$ & cyclopropane & 0.00 & 0.0080 & 0.0079 & -0.0001 & -0.0002 & 3.6 & \\
\hline $\mathrm{C}_{3} \mathrm{H}_{6}$ & propene & 0.01 & 0.0102 & 0.0104 & 0.0009 & 0.0008 & 2.5 & \\
\hline $\mathrm{C}_{3} \mathrm{H}_{8}$ & propane & 0.00 & 0.0084 & 0.0083 & 0.0002 & 0.0002 & 2.5 & \\
\hline $\mathrm{C}_{4} \mathrm{H}_{2}$ & 1,3-butadiyne & 0.21 & 0.0134 & 0.0144 & 0.0024 & 0.0025 & 7.7 & \\
\hline $\mathrm{C}_{4} \mathrm{H}_{4}$ & bicyclo[1.1.0]but-1(3)-ene & 0.68 & 0.0140 & 0.0143 & 0.0054 & 0.0056 & 23.0 & XXX \\
\hline $\mathrm{C}_{4} \mathrm{H}_{4}$ & butatriene & 0.30 & 0.0145 & 0.0146 & 0.0039 & 0.0035 & 10.2 & \\
\hline $\mathrm{C}_{4} \mathrm{H}_{4}$ & cyclobutadiene & 0.48 & 0.0118 & 0.0127 & 0.0024 & 0.0031 & 13.6 & $\mathrm{XX}$ \\
\hline $\mathrm{C}_{4} \mathrm{H}_{4}$ & ethynylethene & 0.09 & 0.0123 & 0.0128 & 0.0018 & 0.0019 & 5.1 & \\
\hline $\mathrm{C}_{4} \mathrm{H}_{4}$ & methylenecyclopropene & 0.00 & 0.0128 & 0.0129 & 0.0034 & 0.0033 & 6.2 & \\
\hline $\mathrm{C}_{4} \mathrm{H}_{4}$ & tetrahedrane & -0.02 & 0.0088 & 0.0088 & 0.0007 & 0.0007 & 11.3 & \\
\hline $\mathrm{C}_{4} \mathrm{H}_{6}$ & 1,2-butadiene & 0.03 & 0.0116 & 0.0117 & 0.0018 & 0.0016 & 4.4 & \\
\hline $\mathrm{C}_{4} \mathrm{H}_{6}$ & 1,3-butadiene & 0.10 & 0.0113 & 0.0116 & 0.0015 & 0.0015 & 6.0 & \\
\hline $\mathrm{C}_{4} \mathrm{H}_{6}$ & 2-butyne & 0.01 & 0.0108 & 0.0110 & 0.0012 & 0.0011 & 1.8 & \\
\hline $\mathrm{C}_{4} \mathrm{H}_{6}$ & bicyclo[1.1.0]butane & 0.00 & 0.0086 & 0.0085 & 0.0004 & 0.0004 & 9.7 & \\
\hline $\mathrm{C}_{4} \mathrm{H}_{6}$ & cyclobutene & 0.01 & 0.0101 & 0.0103 & 0.0014 & 0.0015 & 7.0 & \\
\hline $\mathrm{C}_{4} \mathrm{H}_{6}$ & methylenecyclopropane & 0.02 & 0.0101 & 0.0102 & 0.0014 & 0.0014 & 5.4 & \\
\hline $\mathrm{C}_{4} \mathrm{H}_{8}$ & cyclobutane & -0.02 & 0.0084 & 0.0084 & 0.0003 & 0.0003 & 5.5 & \\
\hline $\mathrm{C}_{4} \mathrm{H}_{8}$ & isobutene & -0.01 & 0.0101 & 0.0102 & 0.0011 & 0.0011 & 4.9 & \\
\hline $\mathrm{C}_{4} \mathrm{H}_{10}$ & isobutane & -0.02 & 0.0087 & 0.0085 & 0.0005 & 0.0004 & 5.4 & \\
\hline $\mathrm{C}_{4} \mathrm{H}_{10}$ & $n$-butane & -0.01 & 0.0085 & 0.0085 & 0.0004 & 0.0003 & 4.0 & \\
\hline $\mathrm{C}_{5} \mathrm{H}_{4}$ & 1,4-pentadiyne & 0.02 & 0.0123 & 0.0129 & 0.0020 & 0.0021 & 2.8 & \\
\hline $\mathrm{C}_{5} \mathrm{H}_{4}$ & pentatetraene & 0.51 & 0.0157 & 0.0158 & 0.0050 & 0.0047 & 13.5 & $\mathrm{X}$ \\
\hline $\mathrm{C}_{5} \mathrm{H}_{4}$ & spiropentadiene & 0.02 & 0.0105 & 0.0112 & 0.0015 & 0.0021 & 11.2 & \\
\hline $\mathrm{C}_{5} \mathrm{H}_{6}$ & [1.1.1]propellane & 0.13 & 0.0106 & 0.0106 & 0.0025 & 0.0025 & 19.9 & \\
\hline $\mathrm{C}_{5} \mathrm{H}_{6}$ & 1,3-cyclopentadiene & 0.03 & 0.0112 & 0.0114 & 0.0021 & 0.0021 & 9.4 & \\
\hline $\mathrm{C}_{5} \mathrm{H}_{6}$ & 3-methylenecyclobutene & 0.07 & 0.0118 & 0.0120 & 0.0027 & 0.0027 & 8.9 & \\
\hline $\mathrm{C}_{5} \mathrm{H}_{6}$ & bicyclo[2.1.0]pent-2-ene & 0.03 & 0.0100 & 0.0102 & 0.0014 & 0.0016 & 11.8 & \\
\hline $\mathrm{C}_{5} \mathrm{H}_{6}$ & cyclopropylacetylene & 0.00 & 0.0104 & 0.0108 & 0.0014 & 0.0016 & 5.4 & \\
\hline $\mathrm{C}_{5} \mathrm{H}_{8}$ & 1,4-pentadiene & 0.00 & 0.0108 & 0.0111 & 0.0015 & 0.0014 & 4.8 & \\
\hline $\mathrm{C}_{5} \mathrm{H}_{8}$ & isoprene & 0.08 & 0.0110 & 0.0111 & 0.0016 & 0.0015 & 7.7 & \\
\hline $\mathrm{C}_{5} \mathrm{H}_{8}$ & spiropentane & -0.04 & 0.0084 & 0.0084 & 0.0003 & 0.0002 & 6.5 & \\
\hline $\mathrm{C}_{5} \mathrm{H}_{10}$ & cyclopentane & -0.05 & 0.0088 & 0.0088 & 0.0007 & 0.0007 & 6.0 & \\
\hline
\end{tabular}


Table S15, contd.

\begin{tabular}{|c|c|c|c|c|c|c|c|c|}
\hline & Molecule & {$[(\mathrm{Q})-(\mathrm{T})] \mathrm{BSE}$} & $T_{1}\left(2_{s d}\right)$ & $T_{1}(4)$ & $\Delta T_{1}\left(2_{s d}\right)$ & $\Delta T_{1}(4)$ & $\% \mathrm{BSE}[(\mathrm{T})]$ & Note \\
\hline $\mathrm{C}_{5} \mathrm{H}_{12}$ & neopentane & -0.05 & 0.0089 & 0.0086 & 0.0008 & 0.0005 & 8.2 & \\
\hline $\mathrm{C}_{5} \mathrm{H}_{12}$ & $n$-pentane & -0.02 & 0.0086 & 0.0086 & 0.0005 & 0.0004 & 4.8 & \\
\hline $\mathrm{C}_{6} \mathrm{H}_{2}$ & 1,3,5-hexatriyne & 0.50 & 0.0139 & 0.0147 & 0.0031 & 0.0032 & 11.8 & \\
\hline $\mathrm{C}_{6} \mathrm{H}_{4}$ & butalene & 0.21 & 0.0132 & 0.0135 & 0.0040 & 0.0041 & 24.1 & \\
\hline $\mathrm{C}_{6} \mathrm{H}_{4}$ & cis-3-hexene-1,5-diyne & 0.21 & 0.0127 & 0.0133 & 0.0023 & 0.0024 & 7.9 & \\
\hline $\mathrm{C}_{6} \mathrm{H}_{4}$ & hexapentaene & 0.98 & 0.0169 & 0.0170 & 0.0063 & 0.0059 & 16.8 & $\mathrm{XX}$ \\
\hline $\mathrm{C}_{6} \mathrm{H}_{4}$ & ortho-benzyne & 0.37 & 0.0127 & 0.0133 & 0.0030 & 0.0032 & 21.9 & \\
\hline $\mathrm{C}_{6} \mathrm{H}_{4}$ & trans-3-hexene-1,5-diyne & 0.22 & 0.0127 & 0.0133 & 0.0023 & 0.0024 & 7.5 & \\
\hline $\mathrm{C}_{6} \mathrm{H}_{4}$ & triafulvalene & 0.06 & 0.0112 & 0.0119 & 0.0020 & 0.0025 & 10.4 & \\
\hline $\mathrm{C}_{6} \mathrm{H}_{6}$ & 2,4-hexadiyne & 0.20 & 0.0120 & 0.0124 & 0.0022 & 0.0021 & 7.1 & \\
\hline $\mathrm{C}_{6} \mathrm{H}_{6}$ & 3,3'-bicyclopropenyl & -0.01 & 0.0101 & 0.0105 & 0.0013 & 0.0016 & 10.0 & \\
\hline $\mathrm{C}_{6} \mathrm{H}_{6}$ & 3,4-dimethylenecyclobutene & 0.16 & 0.0127 & 0.0129 & 0.0033 & 0.0033 & 10.8 & \\
\hline $\mathrm{C}_{6} \mathrm{H}_{6}$ & [3] radialene & 0.41 & 0.0124 & 0.0126 & 0.0031 & 0.0030 & 12.1 & \\
\hline $\mathrm{C}_{6} \mathrm{H}_{6}$ & benzene & -0.19 & 0.0103 & 0.0104 & 0.0010 & 0.0008 & 10.9 & \\
\hline $\mathrm{C}_{6} \mathrm{H}_{6}$ & benzvalene & -0.06 & 0.0098 & 0.0099 & 0.0014 & 0.0014 & 12.8 & \\
\hline $\mathrm{C}_{6} \mathrm{H}_{6}$ & Dewar benzene & -0.01 & 0.0111 & 0.0114 & 0.0023 & 0.0024 & 12.0 & \\
\hline $\mathrm{C}_{6} \mathrm{H}_{6}$ & fulvene & 0.22 & 0.0116 & 0.0118 & 0.0022 & 0.0022 & 11.7 & \\
\hline $\mathrm{C}_{6} \mathrm{H}_{6}$ & prismane & -0.01 & 0.0086 & 0.0086 & 0.0004 & 0.0005 & 12.5 & \\
\hline $\mathrm{C}_{6} \mathrm{H}_{8}$ & 1,3-cyclohexadiene & 0.05 & 0.0108 & 0.0109 & 0.0018 & 0.0018 & 8.4 & \\
\hline $\mathrm{C}_{6} \mathrm{H}_{8}$ & 1,4-cyclohexadiene & -0.03 & 0.0108 & 0.0109 & 0.0019 & 0.0018 & 5.8 & \\
\hline $\mathrm{C}_{6} \mathrm{H}_{8}$ & trans-1,3,5-hexatriene & 0.26 & 0.0116 & 0.0118 & 0.0020 & 0.0019 & 8.7 & \\
\hline $\mathrm{C}_{6} \mathrm{H}_{10}$ & cyclohexene & -0.05 & 0.0099 & 0.0099 & 0.0014 & 0.0013 & 6.2 & \\
\hline $\mathrm{C}_{6} \mathrm{H}_{12}$ & cyclohexane & -0.06 & 0.0089 & 0.0089 & 0.0008 & 0.0007 & 6.3 & \\
\hline $\mathrm{C}_{7} \mathrm{H}_{4}$ & 3,3-diethynylcyclopropene & 0.01 & 0.0120 & 0.0127 & 0.0023 & 0.0026 & 8.1 & \\
\hline $\mathrm{C}_{7} \mathrm{H}_{4}$ & heptahexaene & 1.34 & 0.0177 & 0.0178 & 0.0071 & 0.0067 & 18.7 & $\mathrm{XX}$ \\
\hline $\mathrm{C}_{7} \mathrm{H}_{6}$ & 1,1-diethynylcyclopropane & -0.01 & 0.0115 & 0.0120 & 0.0022 & 0.0023 & 6.9 & \\
\hline $\mathrm{C}_{7} \mathrm{H}_{6}$ & $1,3,5$-norcaratriene & -0.20 & 0.0106 & 0.0106 & 0.0016 & 0.0014 & 14.6 & \\
\hline $\mathrm{C}_{7} \mathrm{H}_{6}$ & fulvenallene & 0.21 & 0.0124 & 0.0126 & 0.0028 & 0.0028 & 12.0 & \\
\hline $\mathrm{C}_{7} \mathrm{H}_{6}$ & spiro[2.4]hepta-1,4,6-triene & 0.02 & 0.0112 & 0.0116 & 0.0022 & 0.0024 & 12.8 & \\
\hline $\mathrm{C}_{7} \mathrm{H}_{8}$ & 1,3,5-cycloheptatriene & 0.10 & 0.0114 & 0.0115 & 0.0022 & 0.0021 & 13.1 & \\
\hline $\mathrm{C}_{7} \mathrm{H}_{8}$ & 2,5-norbornadiene & -0.08 & 0.0109 & 0.0110 & 0.0022 & 0.0021 & 12.8 & \\
\hline $\mathrm{C}_{7} \mathrm{H}_{8}$ & norcaradiene & 0.05 & 0.0107 & 0.0108 & 0.0020 & 0.0020 & 12.4 & \\
\hline $\mathrm{C}_{7} \mathrm{H}_{8}$ & quadricyclane & -0.15 & 0.0093 & 0.0093 & 0.0012 & 0.0011 & 11.6 & \\
\hline $\mathrm{C}_{7} \mathrm{H}_{8}$ & spiro[2.4]hepta-4,6-diene & -0.01 & 0.0106 & 0.0107 & 0.0018 & 0.0019 & 11.9 & \\
\hline $\mathrm{C}_{8} \mathrm{H}_{2}$ & 1,3,5,7-octatetrayne & 0.86 & 0.0141 & 0.0149 & 0.0035 & 0.0036 & 14.3 & \\
\hline $\mathrm{C}_{8} \mathrm{H}_{4}$ & $1,2,3,5,6,7$-cyclooctahexaene & 1.58 & 0.0159 & 0.0162 & 0.0059 & 0.0059 & 21.0 & $\mathrm{XX}$ \\
\hline $\mathrm{C}_{8} \mathrm{H}_{4}$ & 1,5-cyclooctadiene-3,7-diyne & 0.81 & 0.0133 & 0.0140 & 0.0034 & 0.0037 & 16.1 & \\
\hline $\mathrm{C}_{8} \mathrm{H}_{4}$ & octaheptaene & 1.96 & 0.0185 & 0.0185 & 0.0078 & 0.0075 & 20.6 & $\mathrm{XXX}$ \\
\hline $\mathrm{C}_{8} \mathrm{H}_{6}$ & benzocyclobutadiene & 0.10 & 0.0117 & 0.0119 & 0.0024 & 0.0025 & 14.6 & \\
\hline
\end{tabular}


Table S15, contd.

\begin{tabular}{|c|c|c|c|c|c|c|c|c|}
\hline & Molecule & {$[(\mathrm{Q})-(\mathrm{T})] \mathrm{BSE}$} & $T_{1}\left(2_{s d}\right)$ & $T_{1}(4)$ & $\Delta T_{1}\left(2_{s d}\right)$ & $\Delta T_{1}(4)$ & $\% \operatorname{BSE}[(\mathrm{T})]$ & Note \\
\hline $\mathrm{C}_{8} \mathrm{H}_{6}$ & bis(cyclopropa)benzene & -0.22 & 0.0107 & 0.0107 & 0.0019 & 0.0017 & 17.2 & \\
\hline $\mathrm{C}_{8} \mathrm{H}_{6}$ & pentalene & 0.53 & 0.0125 & 0.0127 & 0.0032 & 0.0032 & 15.3 & \\
\hline $\mathrm{C}_{8} \mathrm{H}_{8}$ & 1,3,5,7-cyclooctatetraene & 0.11 & 0.0116 & 0.0118 & 0.0023 & 0.0022 & 10.1 & \\
\hline $\mathrm{C}_{8} \mathrm{H}_{8}$ & benzocyclobutene & -0.26 & 0.0103 & 0.0103 & 0.0013 & 0.0011 & 12.4 & \\
\hline $\mathrm{C}_{8} \mathrm{H}_{8}$ & cubane & 0.00 & 0.0088 & 0.0089 & 0.0007 & 0.0008 & 12.9 & \\
\hline $\mathrm{C}_{8} \mathrm{H}_{8}$ & para-xylylene & 0.65 & 0.0126 & 0.0127 & 0.0032 & 0.0031 & 13.1 & $\mathrm{X}$ \\
\hline $\mathrm{C}_{10} \mathrm{H}_{4}$ & tetraethynylethene & 0.45 & 0.0130 & 0.0136 & 0.0026 & 0.0027 & 11.0 & \\
\hline $\mathrm{C}_{10} \mathrm{H}_{8}$ & naphthalene & -0.23 & 0.0107 & 0.0107 & 0.0015 & 0.0013 & 14.1 & \\
\hline $\mathrm{C}_{10} \mathrm{H}_{8}$ & pentafulvalene & 0.51 & 0.0118 & 0.0120 & 0.0025 & 0.0026 & 14.7 & \\
\hline
\end{tabular}

${ }^{a}[(\mathrm{Q})-(\mathrm{T})] /\left(2,3^{t r}\right)$ bond separation energies in kcal/mol are listed together with $T_{1}$ and excess $T_{1}$ diagnostics $\left(\Delta T_{1}\right.$, see eq. (16)), evaluated from CCSD calculations with $X=2_{s d}$ and $X=4$ basis sets, and the ratio (in \%) between bond separation energy and atomization energy contributions resulting from valence-shell (T) components evaluated for $X=\overline{\mathbf{4}}$. See Section 4.7 for details. Marks in the last column single out those cases where the simple error estimate for neglect of $[(\mathrm{Q})-(\mathrm{T})] /\left(2,3^{t r}\right)$ bond separation energy contributions just matches the actually computed contribution $(\mathrm{X})$, underestimates it by a factor of less than 2 (XX), or by a factor of more than 2 (XXX), see Table S14. 
Table S16: Differences (kcal/mol) between ROHF-based and UHF-based total energies for the carbon atom (triplet ground state $)^{a}$

\begin{tabular}{lccccccc}
\hline Basis set & HF & CCSD & CCSD $(\mathrm{T})$ & CCSDT & CCSDT(Q) & CCSDTQ & CCSDTQ(P) \\
\hline cc-pCVDZ & 2.618 & 0.034 & 0.002 & 0.000 & 0.000 & 0.000 & 0.000 \\
cc-pCVTZ & 3.085 & 0.064 & 0.000 & 0.001 & 0.000 & 0.000 & \\
cc-pCVQZ & 3.151 & 0.069 & -0.002 & 0.002 & 0.001 & & \\
cc-pCV5Z & 3.160 & 0.069 & -0.003 & 0.002 & & & \\
cc-pCV6Z & 3.165 & 0.069 & -0.004 & & & & \\
\hline
\end{tabular}

${ }^{a}$ Coupled-cluster calculations correlate all electrons. ROHF-based coupled-cluster calculations using cc-pCVXZ basis sets were performed using Molpro (keywords UHF-CCSD, UHF-CCSD(T), $X=2 \cdots 6$ ), CFOUR (up to $\operatorname{CCSD}(\mathrm{T}), X=3 \cdots 6$; $\mathrm{CCSDT}, X=3 \cdots 5$ ), and MRCC (up to $\operatorname{CCSDT}(\mathrm{Q}), X=2 \cdots 4$; $\mathrm{CCSDTQ}, X=2 \cdots 3$, CCSDTQ $(\mathrm{P}), X=2)$. UHF-based coupled-cluster calculations were performed using CFOUR and MRCC (for the same method / basis set combinations). Agreement between codes was $0.1 \mu \mathrm{E}_{h}$ or better in all cases. 
Table S17: Differences (kcal/mol) between ROHF-based and UHF-based total energies for the carbon atom (triplet ground state $)^{a}$

\begin{tabular}{lcclccc}
\hline Basis set & HF & CCSD & CCSD(T) & CCSDT & CCSDT(Q) & CCSDTQ \\
\hline cc-pVDZ & 2.589 & 0.054 & 0.023 & 0.021 & 0.021 & 0.021 \\
cc-pVTZ & 3.050 & 0.105 & 0.042 & 0.043 & 0.043 & 0.042 \\
cc-pVQZ & 3.143 & 0.112 & 0.043 & 0.047 & 0.046 & \\
cc-pV5Z & 3.159 & 0.116 & 0.046 & 0.051 & & \\
cc-pV6Z & 3.165 & 0.117 & 0.046 & & & \\
\hline
\end{tabular}

${ }^{a}$ Coupled-cluster calculations correlate valence-electrons only. Both UHF- and ROHF-based coupled-cluster calculations were performed using MRCC (or CFOUR), using semicanonical orbitals for ROHF. 
Table S18: Scalar relativistic contributions to atomization energies $(\mathrm{kcal} / \mathrm{mol})^{a}$

\begin{tabular}{|c|c|c|c|c|c|c|c|c|c|c|}
\hline & Approach & \multicolumn{5}{|c|}{$\mathrm{DKH}$} & \multicolumn{2}{|r|}{ MVD } & \multirow[t]{2}{*}{ BSR } & \multirow[t]{2}{*}{ Unc. } \\
\hline & Basis set & VTZ & VTZ & VTZ & VTZ & VQZ & $-\mathrm{ACV}$ & TZ- & & \\
\hline & Method & $\operatorname{CCSD}(\mathrm{T})$ & CCSD & MP2 & $\mathrm{HF}$ & - & $\operatorname{CSD}(\mathrm{T}$ & - & & \\
\hline $\mathrm{CH}_{4}$ & methane & -0.19 & 0.00 & -0.01 & -0.03 & 0.00 & 0.00 & 0.00 & 0.00 & 0.02 \\
\hline $\mathrm{C}_{2} \mathrm{H}_{2}$ & acetylene & -0.28 & -0.01 & -0.01 & -0.06 & 0.00 & 0.00 & 0.01 & 0.00 & 0.03 \\
\hline $\mathrm{C}_{2} \mathrm{H}_{4}$ & ethylene & -0.33 & -0.01 & -0.01 & -0.06 & 0.00 & 0.00 & 0.00 & 0.00 & 0.03 \\
\hline $\mathrm{C}_{2} \mathrm{H}_{6}$ & ethane & -0.39 & -0.01 & -0.01 & -0.06 & 0.00 & 0.00 & 0.00 & -0.01 & 0.04 \\
\hline $\mathrm{C}_{3} \mathrm{H}_{4}$ & allene & -0.47 & -0.01 & -0.02 & -0.09 & 0.00 & 0.00 & 0.01 & 0.01 & 0.05 \\
\hline $\mathrm{C}_{3} \mathrm{H}_{4}$ & cyclopropene & -0.53 & -0.01 & -0.02 & -0.10 & 0.00 & 0.00 & 0.00 & 0.00 & 0.05 \\
\hline $\mathrm{C}_{3} \mathrm{H}_{4}$ & propyne & -0.48 & -0.01 & -0.01 & -0.09 & 0.00 & 0.00 & 0.01 & 0.01 & 0.05 \\
\hline $\mathrm{C}_{3} \mathrm{H}_{6}$ & cyclopropane & -0.58 & -0.01 & -0.02 & -0.10 & 0.00 & -0.01 & 0.00 & -0.02 & 0.06 \\
\hline $\mathrm{C}_{3} \mathrm{H}_{6}$ & propene & -0.52 & -0.01 & -0.02 & -0.09 & 0.00 & 0.00 & 0.00 & 0.00 & 0.05 \\
\hline $\mathrm{C}_{3} \mathrm{H}_{8}$ & propane & -0.58 & -0.01 & -0.02 & -0.10 & 0.00 & -0.01 & 0.00 & -0.01 & 0.06 \\
\hline $\mathrm{C}_{4} \mathrm{H}_{2}$ & 1,3-butadiyne & -0.59 & -0.02 & -0.02 & -0.12 & 0.00 & -0.01 & 0.02 & 0.03 & 0.06 \\
\hline $\mathrm{C}_{4} \mathrm{H}_{4}$ & bicyclo[1.1.0]but-1(3)-ene & -0.66 & -0.02 & -0.01 & -0.11 & 0.00 & -0.01 & 0.00 & -0.07 & 0.07 \\
\hline $\mathrm{C}_{4} \mathrm{H}_{4}$ & butatriene & -0.63 & -0.02 & -0.02 & -0.12 & 0.00 & -0.01 & 0.02 & 0.03 & 0.06 \\
\hline $\mathrm{C}_{4} \mathrm{H}_{4}$ & cyclobutadiene & -0.65 & -0.02 & -0.02 & -0.13 & 0.00 & -0.01 & 0.01 & -0.01 & 0.07 \\
\hline $\mathrm{C}_{4} \mathrm{H}_{4}$ & ethynylethene & -0.62 & -0.02 & -0.02 & -0.12 & 0.00 & 0.00 & 0.01 & 0.01 & 0.06 \\
\hline $\mathrm{C}_{4} \mathrm{H}_{4}$ & methylenecyclopropene & -0.68 & -0.02 & -0.02 & -0.13 & 0.00 & -0.01 & 0.01 & 0.01 & 0.07 \\
\hline $\mathrm{C}_{4} \mathrm{H}_{4}$ & tetrahedrane & -0.78 & -0.02 & -0.02 & -0.14 & 0.00 & -0.01 & 0.00 & -0.02 & 0.08 \\
\hline $\mathrm{C}_{4} \mathrm{H}_{6}$ & 1,2-butadiene & -0.67 & -0.02 & -0.02 & -0.12 & 0.01 & -0.01 & 0.01 & 0.01 & 0.07 \\
\hline $\mathrm{C}_{4} \mathrm{H}_{6}$ & 1,3-butadiene & -0.66 & -0.02 & -0.02 & -0.12 & 0.01 & 0.00 & 0.01 & 0.00 & 0.07 \\
\hline $\mathrm{C}_{4} \mathrm{H}_{6}$ & 2-butyne & -0.69 & -0.02 & -0.02 & -0.12 & 0.00 & -0.01 & 0.01 & 0.01 & 0.07 \\
\hline $\mathrm{C}_{4} \mathrm{H}_{6}$ & bicyclo[1.1.0]butane & -0.75 & -0.02 & -0.02 & -0.13 & 0.00 & -0.01 & 0.00 & -0.04 & 0.08 \\
\hline $\mathrm{C}_{4} \mathrm{H}_{6}$ & cyclobutene & -0.70 & -0.02 & -0.02 & -0.13 & 0.00 & -0.01 & 0.00 & -0.03 & 0.07 \\
\hline $\mathrm{C}_{4} \mathrm{H}_{6}$ & methylenecyclopropane & -0.72 & -0.02 & -0.03 & -0.13 & 0.01 & -0.01 & 0.01 & -0.01 & 0.07 \\
\hline $\mathrm{C}_{4} \mathrm{H}_{8}$ & cyclobutane & -0.76 & -0.02 & -0.03 & -0.13 & 0.00 & -0.01 & 0.00 & -0.04 & 0.08 \\
\hline $\mathrm{C}_{4} \mathrm{H}_{8}$ & isobutene & -0.71 & -0.02 & -0.03 & -0.12 & 0.01 & -0.01 & 0.00 & -0.01 & 0.07 \\
\hline $\mathrm{C}_{4} \mathrm{H}_{10}$ & isobutane & -0.76 & -0.02 & -0.03 & -0.13 & 0.01 & -0.01 & -0.01 & -0.03 & 0.08 \\
\hline $\mathrm{C}_{4} \mathrm{H}_{10}$ & $n$-butane & -0.77 & -0.02 & -0.03 & -0.13 & 0.01 & -0.01 & -0.01 & -0.02 & 0.08 \\
\hline $\mathrm{C}_{5} \mathrm{H}_{4}$ & 1,4-pentadiyne & -0.78 & -0.02 & -0.02 & -0.15 & 0.01 & -0.01 & 0.02 & 0.02 & 0.08 \\
\hline $\mathrm{C}_{5} \mathrm{H}_{4}$ & pentatetraene & -0.78 & -0.02 & -0.03 & -0.15 & 0.01 & -0.01 & 0.02 & 0.05 & 0.07 \\
\hline $\mathrm{C}_{5} \mathrm{H}_{4}$ & spiropentadiene & -0.87 & -0.03 & -0.02 & -0.17 & 0.00 & -0.01 & 0.01 & 0.00 & 0.09 \\
\hline $\mathrm{C}_{5} \mathrm{H}_{6}$ & [1.1.1]propellane & -0.83 & -0.02 & -0.02 & -0.15 & 0.00 & -0.01 & -0.01 & -0.17 & 0.10 \\
\hline $\mathrm{C}_{5} \mathrm{H}_{6}$ & 1,3-cyclopentadiene & -0.85 & -0.02 & -0.03 & -0.15 & 0.01 & -0.01 & 0.01 & -0.01 & 0.09 \\
\hline $\mathrm{C}_{5} \mathrm{H}_{6}$ & 3-methylenecyclobutene & -0.84 & -0.02 & -0.03 & -0.16 & 0.01 & -0.01 & 0.01 & -0.02 & 0.09 \\
\hline $\mathrm{C}_{5} \mathrm{H}_{6}$ & bicyclo[2.1.0]pent-2-ene & -0.89 & -0.02 & -0.03 & -0.16 & 0.01 & -0.01 & 0.00 & -0.04 & 0.09 \\
\hline $\mathrm{C}_{5} \mathrm{H}_{6}$ & cyclopropylacetylene & -0.87 & -0.02 & -0.03 & -0.16 & 0.01 & -0.01 & 0.01 & -0.01 & 0.09 \\
\hline $\mathrm{C}_{5} \mathrm{H}_{8}$ & 1,4-pentadiene & -0.85 & -0.02 & -0.03 & -0.15 & 0.01 & -0.01 & 0.01 & -0.01 & 0.09 \\
\hline
\end{tabular}


Table S18, contd.

\begin{tabular}{|c|c|c|c|c|c|c|c|c|c|c|}
\hline & Approach & \multicolumn{5}{|c|}{$\mathrm{DKH}$} & \multicolumn{2}{|r|}{ MVD } & \multirow[t]{3}{*}{ BSR } & \multirow[t]{3}{*}{ Unc. } \\
\hline & Basis set & VTZ & VTZ & VTZ & VTZ & VQZ & $-\mathrm{ACV}$ & $\mathrm{TZ}-$ & & \\
\hline & Method & $\operatorname{CCSD}(\mathrm{T})$ & CCSD & MP2 & $\mathrm{HF}$ & - & $\operatorname{CSD}(\mathrm{T}$ & - & & \\
\hline $\mathrm{C}_{5} \mathrm{H}_{8}$ & isoprene & -0.85 & -0.02 & -0.03 & -0.15 & 0.01 & -0.01 & 0.01 & -0.01 & 0.09 \\
\hline $\mathrm{C}_{5} \mathrm{H}_{8}$ & spiropentane & -0.97 & -0.02 & -0.03 & -0.16 & 0.01 & -0.01 & 0.00 & -0.03 & 0.10 \\
\hline $\mathrm{C}_{5} \mathrm{H}_{10}$ & cyclopentane & -0.95 & -0.02 & -0.04 & -0.16 & 0.01 & -0.01 & -0.01 & -0.04 & 0.10 \\
\hline $\mathrm{C}_{5} \mathrm{H}_{12}$ & neopentane & -0.95 & -0.02 & -0.03 & -0.16 & 0.01 & -0.01 & -0.01 & -0.04 & 0.10 \\
\hline $\mathrm{C}_{5} \mathrm{H}_{12}$ & $n$-pentane & -0.96 & -0.02 & -0.03 & -0.16 & 0.01 & -0.01 & -0.01 & -0.03 & 0.10 \\
\hline $\mathrm{C}_{6} \mathrm{H}_{2}$ & 1,3,5-hexatriyne & -0.90 & -0.03 & -0.02 & -0.19 & 0.01 & -0.01 & 0.03 & 0.06 & 0.08 \\
\hline $\mathrm{C}_{6} \mathrm{H}_{4}$ & butalene & -0.98 & -0.03 & -0.03 & -0.20 & 0.00 & -0.01 & 0.01 & -0.02 & 0.10 \\
\hline $\mathrm{C}_{6} \mathrm{H}_{4}$ & cis-3-hexene-1,5-diyne & -0.92 & -0.03 & -0.03 & -0.18 & 0.01 & -0.01 & 0.02 & 0.02 & 0.09 \\
\hline $\mathrm{C}_{6} \mathrm{H}_{4}$ & hexapentaene & -0.93 & -0.03 & -0.03 & -0.18 & 0.01 & -0.01 & 0.03 & 0.07 & 0.09 \\
\hline $\mathrm{C}_{6} \mathrm{H}_{4}$ & ortho-benzyne & -0.97 & -0.03 & -0.02 & -0.19 & 0.01 & -0.01 & 0.01 & 0.02 & 0.09 \\
\hline $\mathrm{C}_{6} \mathrm{H}_{4}$ & trans-3-hexene-1,5-diyne & -0.91 & -0.03 & -0.03 & -0.18 & 0.01 & -0.01 & 0.02 & 0.02 & 0.09 \\
\hline $\mathrm{C}_{6} \mathrm{H}_{4}$ & triafulvalene & -1.04 & -0.03 & -0.03 & -0.20 & 0.01 & -0.01 & 0.02 & 0.04 & 0.10 \\
\hline $\mathrm{C}_{6} \mathrm{H}_{6}$ & 2,4-hexadiyne & -0.99 & -0.03 & -0.03 & -0.18 & 0.01 & -0.01 & 0.02 & 0.04 & 0.10 \\
\hline $\mathrm{C}_{6} \mathrm{H}_{6}$ & 3,3'-bicyclopropenyl & -1.05 & -0.03 & -0.03 & -0.20 & 0.01 & -0.01 & 0.01 & -0.02 & 0.11 \\
\hline $\mathrm{C}_{6} \mathrm{H}_{6}$ & 3,4-dimethylenecyclobutene & -0.98 & -0.03 & -0.03 & -0.19 & 0.01 & -0.01 & 0.01 & -0.02 & 0.10 \\
\hline $\mathrm{C}_{6} \mathrm{H}_{6}$ & [3]radialene & -1.01 & -0.03 & -0.03 & -0.19 & 0.01 & -0.01 & 0.02 & 0.01 & 0.10 \\
\hline $\mathrm{C}_{6} \mathrm{H}_{6}$ & benzene & -1.00 & -0.03 & -0.03 & -0.18 & 0.01 & -0.01 & 0.01 & 0.00 & 0.10 \\
\hline $\mathrm{C}_{6} \mathrm{H}_{6}$ & benzvalene & -1.07 & -0.03 & -0.03 & -0.20 & 0.01 & -0.01 & 0.00 & -0.07 & 0.11 \\
\hline $\mathrm{C}_{6} \mathrm{H}_{6}$ & Dewar benzene & -1.02 & -0.03 & -0.03 & -0.19 & 0.01 & -0.01 & 0.00 & -0.05 & 0.11 \\
\hline $\mathrm{C}_{6} \mathrm{H}_{6}$ & fulvene & -0.99 & -0.03 & -0.03 & -0.18 & 0.01 & -0.01 & 0.01 & -0.01 & 0.10 \\
\hline $\mathrm{C}_{6} \mathrm{H}_{6}$ & prismane & -1.11 & -0.03 & -0.03 & -0.20 & 0.00 & -0.01 & 0.00 & -0.09 & 0.12 \\
\hline $\mathrm{C}_{6} \mathrm{H}_{8}$ & 1,3-cyclohexadiene & -1.05 & -0.03 & -0.04 & -0.18 & 0.01 & -0.01 & 0.00 & -0.01 & 0.11 \\
\hline $\mathrm{C}_{6} \mathrm{H}_{8}$ & 1,4-cyclohexadiene & -1.06 & -0.03 & -0.04 & -0.18 & 0.01 & -0.01 & 0.01 & -0.01 & 0.11 \\
\hline $\mathrm{C}_{6} \mathrm{H}_{8}$ & trans-1,3,5-hexatriene & -0.99 & -0.03 & -0.03 & -0.18 & 0.01 & -0.01 & 0.01 & 0.00 & 0.10 \\
\hline $\mathrm{C}_{6} \mathrm{H}_{10}$ & cyclohexene & -1.10 & -0.02 & -0.04 & -0.19 & 0.01 & -0.01 & 0.00 & -0.02 & 0.11 \\
\hline $\mathrm{C}_{6} \mathrm{H}_{12}$ & cyclohexane & -1.15 & -0.02 & -0.04 & -0.19 & 0.01 & -0.01 & -0.01 & -0.04 & 0.12 \\
\hline $\mathrm{C}_{7} \mathrm{H}_{4}$ & 3,3-diethynylcyclopropene & -1.11 & -0.03 & -0.03 & -0.22 & & & & 0.01 & 0.11 \\
\hline $\mathrm{C}_{7} \mathrm{H}_{4}$ & heptahexaene & -1.08 & -0.03 & -0.03 & -0.21 & & & & 0.09 & 0.10 \\
\hline $\mathrm{C}_{7} \mathrm{H}_{6}$ & 1,1-diethynylcyclopropane & -1.16 & -0.03 & -0.04 & -0.22 & & & & 0.00 & 0.12 \\
\hline $\mathrm{C}_{7} \mathrm{H}_{6}$ & 1,3,5-norcaratriene & -1.21 & -0.03 & -0.04 & -0.22 & & & & 0.01 & 0.12 \\
\hline $\mathrm{C}_{7} \mathrm{H}_{6}$ & fulvenallene & -1.13 & -0.03 & -0.04 & -0.21 & & & & 0.00 & 0.11 \\
\hline $\mathrm{C}_{7} \mathrm{H}_{6}$ & spiro[2.4]hepta-1,4,6-triene & -1.18 & -0.03 & -0.04 & -0.22 & & & & -0.02 & 0.12 \\
\hline $\mathrm{C}_{7} \mathrm{H}_{8}$ & 1,3,5-cycloheptatriene & -1.19 & -0.03 & -0.04 & -0.21 & & & & -0.01 & 0.12 \\
\hline $\mathrm{C}_{7} \mathrm{H}_{8}$ & 2,5 -norbornadiene & -1.21 & -0.03 & -0.04 & -0.22 & & & & -0.05 & 0.13 \\
\hline $\mathrm{C}_{7} \mathrm{H}_{8}$ & norcaradiene & -1.24 & -0.03 & -0.04 & -0.22 & & & & -0.02 & 0.13 \\
\hline $\mathrm{C}_{7} \mathrm{H}_{8}$ & quadricyclane & -1.32 & -0.03 & -0.04 & -0.23 & & & & -0.09 & 0.14 \\
\hline $\mathrm{C}_{7} \mathrm{H}_{8}$ & spiro[2.4]hepta-4,6-diene & -1.23 & -0.03 & -0.04 & -0.22 & & & & -0.03 & 0.13 \\
\hline
\end{tabular}


Table S18, contd.

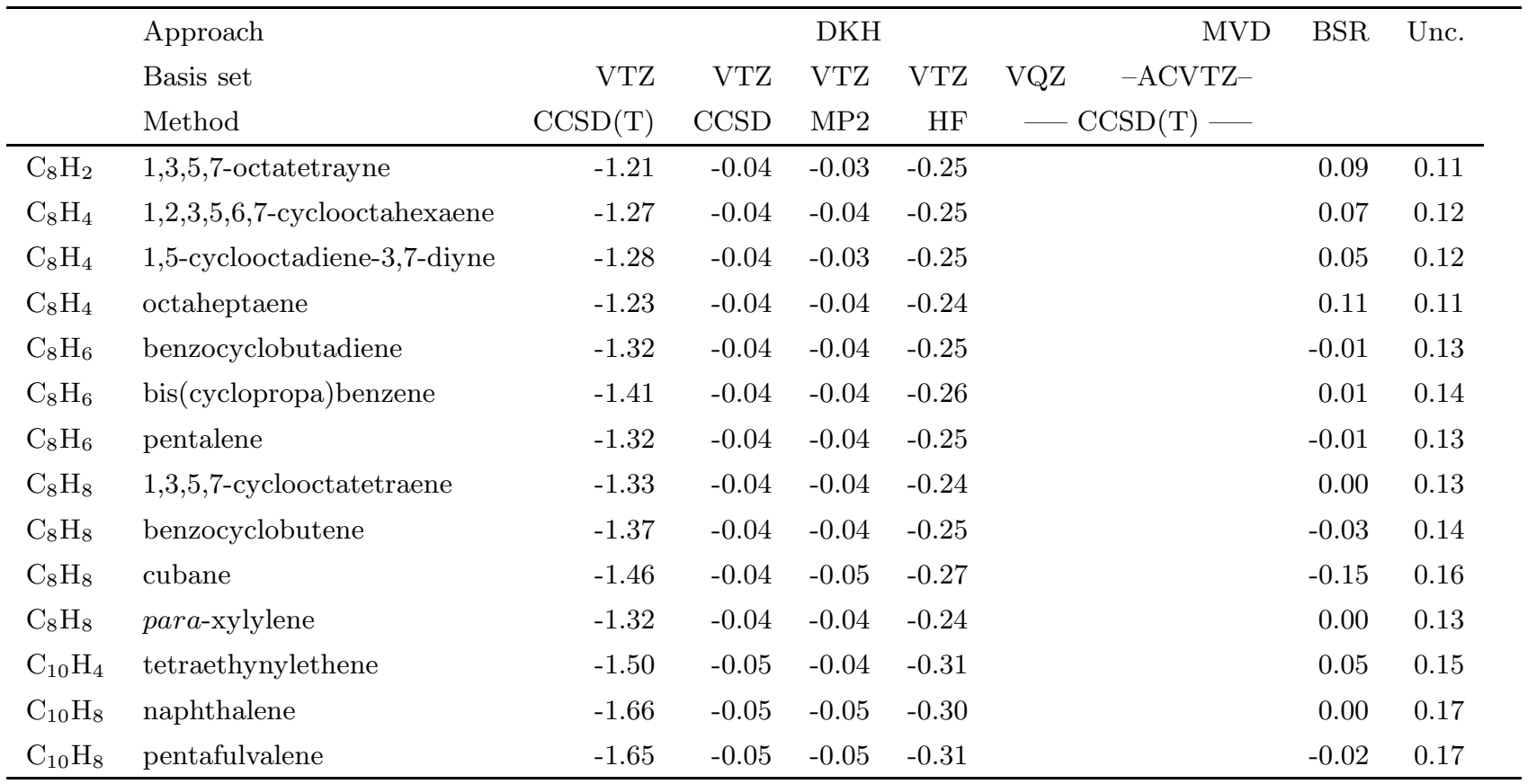

${ }^{a}$ All numbers in data columns 2 to 8 are expressed as deviation from the value reported in column 1 . Column 9 ("Unc.") reports the uncertainty estimate for ATOMIC(hc) evaluations from bond increments ("BSR") as $10 \%$ of the total value (see text and Table 1). Basis set designations are short-hand for cc-pVTZ, cc-pVQZ, and aug-cc-pCVTZ (non-relativistic hamiltonians) and cc-pVTZ-DK, cc-pVQZ-DK, and aug-cc-pCVTZ-DK (DKH hamiltonians). Correlation treatments using ACVTZ basis sets consider all electrons. Note that bond increments (column 8, "BSR") were derived from MVD calculations ${ }^{54}$ at the CCSD(T)(full)/ACVTZ level (column 7). The slight disagreement between BSR and MVD values for BSR prototype molecules is due to roundoff error in the bond increments. 
Table S19: $D B O C$ contributions to atomization energies $(\mathrm{kcal} / \mathrm{mol})^{a}$

\begin{tabular}{|c|c|c|c|c|c|c|c|}
\hline & & \multirow{3}{*}{$\begin{array}{r}\text { Reference } \\
\text { CCSD }\end{array}$} & \multicolumn{4}{|c|}{ Deviation } & \multirow{3}{*}{ Unc. } \\
\hline & & & $\mathrm{HF}$ & BSR & BSR & EMP & \\
\hline & & & & $\mathrm{HF}$ & CCSD & CCSD & \\
\hline $\mathrm{CH}_{4}$ & methane & 0.060 & 0.05 & 0.05 & 0.00 & -0.01 & 0.02 \\
\hline $\mathrm{C}_{2} \mathrm{H}_{2}$ & acetylene & 0.078 & 0.04 & 0.04 & 0.00 & -0.03 & 0.03 \\
\hline $\mathrm{C}_{2} \mathrm{H}_{4}$ & ethylene & 0.060 & 0.06 & 0.06 & 0.00 & 0.01 & 0.03 \\
\hline $\mathrm{C}_{2} \mathrm{H}_{6}$ & ethane & 0.079 & 0.08 & 0.07 & 0.00 & 0.00 & 0.04 \\
\hline $\mathrm{C}_{3} \mathrm{H}_{4}$ & allene & 0.090 & 0.07 & 0.05 & -0.03 & 0.00 & 0.04 \\
\hline $\mathrm{C}_{3} \mathrm{H}_{4}$ & cyclopropene & 0.084 & 0.06 & 0.02 & -0.05 & 0.00 & 0.04 \\
\hline $\mathrm{C}_{3} \mathrm{H}_{4}$ & propyne & 0.110 & 0.07 & 0.05 & -0.01 & -0.03 & 0.04 \\
\hline $\mathrm{C}_{3} \mathrm{H}_{6}$ & cyclopropane & 0.119 & 0.08 & 0.02 & -0.06 & -0.03 & 0.04 \\
\hline $\mathrm{C}_{3} \mathrm{H}_{6}$ & propene & 0.098 & 0.09 & 0.07 & -0.02 & 0.00 & 0.05 \\
\hline $\mathrm{C}_{3} \mathrm{H}_{8}$ & propane & 0.109 & 0.10 & 0.09 & -0.01 & 0.00 & 0.05 \\
\hline $\mathrm{C}_{4} \mathrm{H}_{2}$ & 1,3-butadiyne & 0.115 & 0.06 & 0.06 & 0.00 & -0.03 & 0.04 \\
\hline $\mathrm{C}_{4} \mathrm{H}_{4}$ & bicyclo[1.1.0]but-1(3)-ene & 0.070 & 0.05 & 0.02 & -0.06 & 0.02 & 0.05 \\
\hline $\mathrm{C}_{4} \mathrm{H}_{4}$ & butatriene & 0.090 & 0.08 & 0.06 & -0.03 & 0.02 & 0.05 \\
\hline $\mathrm{C}_{4} \mathrm{H}_{4}$ & cyclobutadiene & 0.100 & 0.07 & 0.02 & -0.06 & 0.00 & 0.05 \\
\hline $\mathrm{C}_{4} \mathrm{H}_{4}$ & ethynylethene & 0.118 & 0.08 & 0.06 & -0.02 & -0.02 & 0.05 \\
\hline $\mathrm{C}_{4} \mathrm{H}_{4}$ & methylenecyclopropene & 0.117 & 0.07 & 0.00 & -0.08 & -0.02 & 0.05 \\
\hline $\mathrm{C}_{4} \mathrm{H}_{4}$ & tetrahedrane & 0.169 & 0.07 & -0.11 & -0.18 & -0.08 & 0.04 \\
\hline $\mathrm{C}_{4} \mathrm{H}_{6}$ & 1,2-butadiene & 0.122 & 0.09 & 0.06 & -0.04 & 0.00 & 0.06 \\
\hline $\mathrm{C}_{4} \mathrm{H}_{6}$ & 1,3-butadiene & 0.112 & 0.10 & 0.07 & -0.03 & 0.01 & 0.06 \\
\hline $\mathrm{C}_{4} \mathrm{H}_{6}$ & 2-butyne & 0.136 & 0.09 & 0.07 & -0.02 & -0.03 & 0.06 \\
\hline $\mathrm{C}_{4} \mathrm{H}_{6}$ & bicyclo[1.1.0]butane & 0.138 & 0.09 & -0.02 & -0.11 & -0.04 & 0.05 \\
\hline $\mathrm{C}_{4} \mathrm{H}_{6}$ & cyclobutene & 0.112 & 0.09 & 0.04 & -0.06 & 0.00 & 0.06 \\
\hline $\mathrm{C}_{4} \mathrm{H}_{6}$ & methylenecyclopropane & 0.133 & 0.09 & 0.02 & -0.08 & -0.02 & 0.06 \\
\hline $\mathrm{C}_{4} \mathrm{H}_{8}$ & cyclobutane & 0.123 & 0.11 & 0.06 & -0.05 & 0.00 & 0.06 \\
\hline $\mathrm{C}_{4} \mathrm{H}_{8}$ & isobutene & 0.141 & 0.11 & 0.07 & -0.04 & -0.01 & 0.06 \\
\hline $\mathrm{C}_{4} \mathrm{H}_{10}$ & isobutane & 0.149 & 0.13 & 0.09 & -0.03 & -0.01 & 0.07 \\
\hline $\mathrm{C}_{4} \mathrm{H}_{10}$ & $n$-butane & 0.139 & 0.13 & 0.10 & -0.02 & 0.00 & 0.07 \\
\hline $\mathrm{C}_{5} \mathrm{H}_{4}$ & 1,4-pentadiyne & 0.161 & 0.08 & 0.06 & -0.03 & -0.05 & 0.06 \\
\hline $\mathrm{C}_{5} \mathrm{H}_{4}$ & pentatetraene & 0.107 & 0.08 & 0.06 & -0.05 & 0.02 & 0.06 \\
\hline $\mathrm{C}_{5} \mathrm{H}_{4}$ & spiropentadiene & 0.131 & 0.08 & -0.03 & -0.12 & -0.02 & 0.06 \\
\hline $\mathrm{C}_{5} \mathrm{H}_{6}$ & [1.1.1]propellane & 0.159 & 0.09 & -0.06 & -0.15 & -0.04 & 0.06 \\
\hline $\mathrm{C}_{5} \mathrm{H}_{6}$ & 1,3-cyclopentadiene & 0.152 & 0.10 & 0.01 & -0.10 & -0.02 & 0.07 \\
\hline $\mathrm{C}_{5} \mathrm{H}_{6}$ & 3-methylenecyclobutene & 0.140 & 0.10 & 0.02 & -0.09 & -0.01 & 0.07 \\
\hline $\mathrm{C}_{5} \mathrm{H}_{6}$ & bicyclo[2.1.0]pent-2-ene & 0.154 & 0.10 & -0.02 & -0.12 & -0.03 & 0.06 \\
\hline $\mathrm{C}_{5} \mathrm{H}_{6}$ & cyclopropylacetylene & 0.178 & 0.10 & 0.01 & -0.09 & -0.06 & 0.06 \\
\hline $\mathrm{C}_{5} \mathrm{H}_{8}$ & 1,4-pentadiene & 0.145 & 0.13 & 0.08 & -0.05 & 0.00 & 0.07 \\
\hline
\end{tabular}


Table S19, contd.

\begin{tabular}{|c|c|c|c|c|c|c|c|}
\hline & & \multirow{3}{*}{$\begin{array}{r}\text { Reference } \\
\text { CCSD }\end{array}$} & \multicolumn{4}{|c|}{ Deviation } & \multirow{3}{*}{ Unc. } \\
\hline & & & $\mathrm{HF}$ & BSR & BSR & EMP & \\
\hline & & & & $\mathrm{HF}$ & CCSD & CCSD & \\
\hline $\mathrm{C}_{5} \mathrm{H}_{8}$ & isoprene & 0.155 & 0.13 & 0.07 & -0.06 & -0.01 & 0.07 \\
\hline $\mathrm{C}_{5} \mathrm{H}_{8}$ & spiropentane & 0.183 & 0.12 & -0.02 & -0.13 & -0.05 & 0.07 \\
\hline $\mathrm{C}_{5} \mathrm{H}_{10}$ & cyclopentane & 0.152 & 0.13 & 0.07 & -0.06 & 0.00 & 0.07 \\
\hline $\mathrm{C}_{5} \mathrm{H}_{12}$ & neopentane & 0.196 & 0.15 & 0.09 & -0.06 & -0.03 & 0.08 \\
\hline $\mathrm{C}_{5} \mathrm{H}_{12}$ & $n$-pentane & 0.168 & 0.16 & 0.12 & -0.04 & 0.00 & 0.08 \\
\hline $\mathrm{C}_{6} \mathrm{H}_{2}$ & 1,3,5-hexatriyne & 0.148 & 0.07 & 0.08 & 0.00 & -0.03 & 0.06 \\
\hline $\mathrm{C}_{6} \mathrm{H}_{4}$ & butalene & 0.092 & 0.07 & 0.02 & -0.08 & 0.04 & 0.07 \\
\hline $\mathrm{C}_{6} \mathrm{H}_{4}$ & cis-3-hexene-1,5-diyne & 0.169 & 0.09 & 0.06 & -0.04 & -0.04 & 0.07 \\
\hline $\mathrm{C}_{6} \mathrm{H}_{4}$ & hexapentaene & 0.110 & 0.09 & 0.07 & -0.05 & 0.04 & 0.07 \\
\hline $\mathrm{C}_{6} \mathrm{H}_{4}$ & ortho-benzyne & 0.116 & 0.06 & 0.06 & -0.05 & 0.02 & 0.07 \\
\hline $\mathrm{C}_{6} \mathrm{H}_{4}$ & trans-3-hexene-1,5-diyne & 0.172 & 0.09 & 0.06 & -0.04 & -0.04 & 0.07 \\
\hline $\mathrm{C}_{6} \mathrm{H}_{4}$ & triafulvalene & 0.156 & 0.09 & -0.04 & -0.15 & -0.02 & 0.07 \\
\hline $\mathrm{C}_{6} \mathrm{H}_{6}$ & 2,4-hexadiyne & 0.177 & 0.10 & 0.09 & -0.03 & -0.04 & 0.07 \\
\hline $\mathrm{C}_{6} \mathrm{H}_{6}$ & 3,3'-bicyclopropenyl & 0.166 & 0.10 & -0.02 & -0.14 & -0.02 & 0.07 \\
\hline $\mathrm{C}_{6} \mathrm{H}_{6}$ & 3,4-dimethylenecyclobutene & 0.161 & 0.11 & 0.02 & -0.11 & -0.01 & 0.07 \\
\hline $\mathrm{C}_{6} \mathrm{H}_{6}$ & [3]radialene & 0.146 & 0.11 & 0.03 & -0.09 & 0.00 & 0.07 \\
\hline $\mathrm{C}_{6} \mathrm{H}_{6}$ & benzene & 0.139 & 0.10 & 0.04 & -0.09 & 0.01 & 0.07 \\
\hline $\mathrm{C}_{6} \mathrm{H}_{6}$ & benzvalene & 0.190 & 0.10 & -0.08 & -0.18 & -0.06 & 0.07 \\
\hline $\mathrm{C}_{6} \mathrm{H}_{6}$ & Dewar benzene & 0.161 & 0.10 & -0.02 & -0.13 & -0.02 & 0.07 \\
\hline $\mathrm{C}_{6} \mathrm{H}_{6}$ & fulvene & 0.160 & 0.11 & 0.02 & -0.11 & -0.01 & 0.07 \\
\hline $\mathrm{C}_{6} \mathrm{H}_{6}$ & prismane & 0.189 & 0.10 & -0.11 & -0.21 & -0.06 & 0.06 \\
\hline $\mathrm{C}_{6} \mathrm{H}_{8}$ & 1,3-cyclohexadiene & 0.161 & 0.13 & 0.05 & -0.09 & 0.00 & 0.08 \\
\hline $\mathrm{C}_{6} \mathrm{H}_{8}$ & 1,4-cyclohexadiene & 0.158 & 0.13 & 0.05 & -0.09 & 0.00 & 0.08 \\
\hline $\mathrm{C}_{6} \mathrm{H}_{8}$ & trans-1,3,5-hexatriene & 0.161 & 0.14 & 0.08 & -0.07 & 0.01 & 0.08 \\
\hline $\mathrm{C}_{6} \mathrm{H}_{10}$ & cyclohexene & 0.171 & 0.14 & 0.07 & -0.08 & 0.00 & 0.09 \\
\hline $\mathrm{C}_{6} \mathrm{H}_{12}$ & cyclohexane & 0.179 & 0.16 & 0.09 & -0.07 & 0.00 & 0.09 \\
\hline $\mathrm{C}_{7} \mathrm{H}_{4}$ & 3,3-diethynylcyclopropene & 0.208 & 0.09 & 0.01 & -0.10 & -0.06 & 0.07 \\
\hline $\mathrm{C}_{7} \mathrm{H}_{4}$ & heptahexaene & 0.122 & 0.09 & 0.07 & -0.07 & 0.05 & 0.08 \\
\hline $\mathrm{C}_{7} \mathrm{H}_{6}$ & 1,1-diethynylcyclopropane & 0.234 & 0.11 & 0.01 & -0.11 & -0.08 & 0.08 \\
\hline $\mathrm{C}_{7} \mathrm{H}_{6}$ & 1,3,5-norcaratriene & 0.153 & 0.10 & 0.01 & -0.12 & 0.01 & 0.08 \\
\hline $\mathrm{C}_{7} \mathrm{H}_{6}$ & fulvenallene & 0.186 & 0.11 & 0.00 & -0.13 & -0.02 & 0.09 \\
\hline $\mathrm{C}_{7} \mathrm{H}_{6}$ & spiro[2.4]hepta-1,4,6-triene & 0.197 & 0.11 & -0.04 & -0.17 & -0.03 & 0.08 \\
\hline $\mathrm{C}_{7} \mathrm{H}_{8}$ & 1,3,5-cycloheptatriene & 0.174 & 0.13 & 0.05 & -0.10 & 0.01 & 0.09 \\
\hline $\mathrm{C}_{7} \mathrm{H}_{8}$ & 2,5 -norbornadiene & 0.202 & 0.13 & -0.01 & -0.16 & -0.03 & 0.09 \\
\hline $\mathrm{C}_{7} \mathrm{H}_{8}$ & norcaradiene & 0.195 & 0.13 & 0.00 & -0.15 & -0.02 & 0.09 \\
\hline $\mathrm{C}_{7} \mathrm{H}_{8}$ & quadricyclane & 0.233 & 0.12 & -0.11 & -0.23 & -0.08 & 0.08 \\
\hline $\mathrm{C}_{7} \mathrm{H}_{8}$ & spiro[2.4]hepta-4,6-diene & 0.217 & 0.13 & -0.03 & -0.17 & -0.04 & 0.09 \\
\hline
\end{tabular}


Table S19, contd.

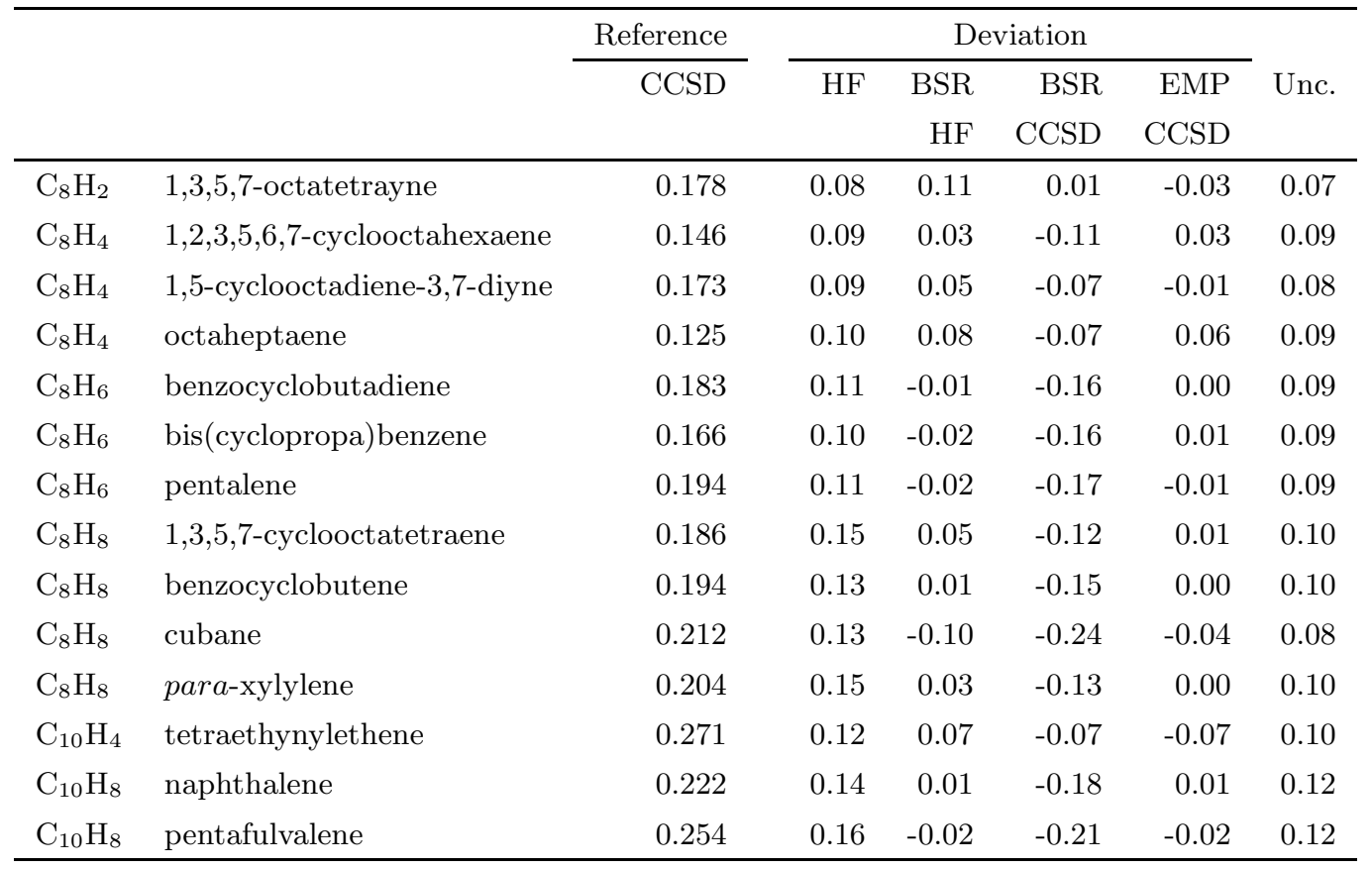

${ }^{a}$ Reference calculations are at the CCSD(full)/cc-pCVDZ level. Deviations from this reference are given for $\mathrm{HF} / \mathrm{cc}-\mathrm{pVDZ}$, the BSR model derived from HF/cc-pVDZ (used in ATOMIC ${ }^{54}$ ) and the BSR and the empirical ("EMP") models derived here from CCSD(full)/cc-pCVDZ data. The last column ("Unc.") reports the 50\% uncertainty estimated for the empirical model. 
Table S20: DBOC for homologous series of hydrocarbons $(\mathrm{kcal} / \mathrm{mol})^{a}$

\begin{tabular}{|c|c|c|c|c|c|}
\hline \multirow[t]{4}{*}{$n$} & n-alkanes & cycloalkanes & $n$-alkanes & polyenes & polyynes \\
\hline & & & vs cycloalkanes & & \\
\hline & $\mathrm{E}\left(\mathrm{C}_{n} \mathrm{H}_{2 n+2}\right)$ & $\mathrm{E}\left(\mathrm{C}_{n} \mathrm{H}_{2 n}\right)$ & $\mathrm{E}\left(\mathrm{C}_{n} \mathrm{H}_{2 n+2}\right)$ & $\mathrm{E}\left(\mathrm{C}_{n} \mathrm{H}_{n+2}\right)$ & $\mathrm{E}\left(\mathrm{C}_{n} \mathrm{H}_{2}\right)$ \\
\hline & $-\mathrm{E}\left(\mathrm{C}_{n-1} \mathrm{H}_{2 n}\right)$ & $-\mathrm{E}\left(\mathrm{C}_{n-1} \mathrm{H}_{2 n-2}\right)$ & $-\mathrm{E}\left(\mathrm{C}_{n} \mathrm{H}_{2 n}\right)$ & $-\mathrm{E}\left(\mathrm{C}_{n-2} \mathrm{H}_{n}\right)$ & $\mathrm{E}\left(\mathrm{C}_{n-2} \mathrm{H}_{2}\right)$ \\
\hline 3 & 0.030 & & -0.010 & & \\
\hline 4 & 0.030 & 0.004 & 0.016 & 0.052 & 0.037 \\
\hline 5 & 0.029 & 0.029 & 0.016 & & \\
\hline 6 & 0.029 & 0.027 & 0.018 & 0.049 & 0.033 \\
\hline 7 & 0.030 & & & & \\
\hline 8 & & & & 0.048 & 0.030 \\
\hline \multicolumn{6}{|l|}{9} \\
\hline 10 & & & & & 0.028 \\
\hline avg & \multicolumn{2}{|c|}{$0.029^{b}$} & $0.017^{b}$ & 0.050 & 0.032 \\
\hline label $^{c}$ & \multicolumn{2}{|c|}{ A } & B & $\mathrm{C}$ & $\mathrm{D}$ \\
\hline bonds $^{d}$ & \multicolumn{2}{|c|}{${ }^{3} \mathrm{C}-\mathrm{C}^{3}+2{ }^{3} \mathrm{C}-\mathrm{H}$} & ${ }_{-}^{3} \mathrm{C}-\mathrm{C}^{3}+2{ }^{3} \mathrm{C}-\mathrm{H}$ & ${ }^{2} \mathrm{C}=\mathrm{C}^{2}+{ }^{3} \mathrm{C}-\mathrm{C}^{3}+2{ }^{3} \mathrm{C}-\mathrm{H}$ & ${ }^{1} \mathrm{C} \equiv \mathrm{C}^{1}+{ }^{3} \mathrm{C}-\mathrm{C}^{3}$ \\
\hline
\end{tabular}

${ }^{a}$ DBOC differences have been computed from DBOC contributions to atomization energies at the CCSD(full)/ccpCVDZ level, see Table S19. Calculations have also been performed for four molecules outside the original 87molecule benchmark set $(n$-hexane $(0.197 \mathrm{kcal} / \mathrm{mol}) ; n$-heptane $(0.227 \mathrm{kcal} / \mathrm{mol}) ;$ all-trans-1,3,5,7-octatetraene $(0.209 \mathrm{kcal} / \mathrm{mol}) ; 1,3,5,7,9$-decapentayne $(0.206 \mathrm{kcal} / \mathrm{mol}))$ in order to increase the number of possible comparisons. ${ }^{b}$ The average excludes values derived from data for cyclopropane; see text.

${ }^{c}$ Label as used in the text, Section 4.11 .

${ }^{d}$ Number and type of bonds by which the compared species differ. 
Table S21: Atomization energies of small hydrocarbons

\begin{tabular}{|c|c|c|c|c|c|c|c|c|c|c|}
\hline & \multirow[t]{2}{*}{ Molecule } & \multirow[t]{2}{*}{$\mathrm{MID}^{a}$} & \multicolumn{3}{|c|}{$E_{A, e^{b}}^{b}$} & \multirow[t]{2}{*}{$\Delta T_{1}\left(2_{s d}\right)^{c}$} & \multicolumn{4}{|c|}{ ATOMIC(hc) - ATOMIC $^{d}$} \\
\hline & & & Best. est. & ATOMIC & ATOMIC(hc) & & $\operatorname{CCSD}(\mathrm{T})$ & h.o. & DBOC & sum \\
\hline $\mathrm{CH}_{4}$ & methane & 1 & $420.17 \pm 0.06$ & 420.29 & $420.12 \pm 0.10$ & 0.0000 & -0.12 & 0.01 & -0.06 & -0.17 \\
\hline $\mathrm{C}_{2} \mathrm{H}_{2}$ & acetylene & 2 & $404.98 \pm 0.10$ & 404.94 & $404.93 \pm 0.13$ & 0.0000 & -0.22 & 0.28 & -0.07 & -0.02 \\
\hline $\mathrm{C}_{2} \mathrm{H}_{4}$ & ethylene & 3 & $563.57 \pm 0.10$ & 563.67 & $563.55 \pm 0.15$ & 0.0000 & -0.22 & 0.15 & -0.05 & -0.12 \\
\hline $\mathrm{C}_{2} \mathrm{H}_{6}$ & ethane & 4 & $712.52 \pm 0.11$ & 712.73 & $712.47 \pm 0.18$ & 0.0000 & -0.23 & 0.05 & -0.07 & -0.26 \\
\hline $\mathrm{C}_{3} \mathrm{H}_{4}$ & allene & 5 & $703.40 \pm 0.16$ & 703.47 & $703.40 \pm 0.33$ & 0.0018 & -0.32 & 0.30 & -0.05 & -0.07 \\
\hline $\mathrm{C}_{3} \mathrm{H}_{4}$ & cyclopropene & 6 & $681.15 \pm 0.18$ & 681.25 & $681.14 \pm 0.23$ & 0.0009 & -0.31 & 0.23 & -0.02 & -0.11 \\
\hline $\mathrm{C}_{3} \mathrm{H}_{4}$ & propyne & 7 & $704.74 \pm 0.17$ & 704.77 & $704.68 \pm 0.22$ & 0.0010 & -0.33 & 0.32 & -0.08 & -0.10 \\
\hline $\mathrm{C}_{3} \mathrm{H}_{6}$ & cyclopropane & 8 & $853.17 \pm 0.19$ & 853.33 & $853.08 \pm 0.26$ & -0.0001 & -0.32 & 0.12 & -0.04 & -0.24 \\
\hline $\mathrm{C}_{3} \mathrm{H}_{6}$ & propene & 9 & $860.75 \pm 0.17$ & 860.91 & $860.70 \pm 0.24$ & 0.0009 & -0.33 & 0.19 & -0.07 & -0.21 \\
\hline $\mathrm{C}_{3} \mathrm{H}_{8}$ & propane & 10 & $1007.03 \pm 0.18$ & 1007.30 & $1006.96 \pm 0.26$ & 0.0002 & -0.34 & 0.09 & -0.09 & -0.34 \\
\hline $\mathrm{C}_{4} \mathrm{H}_{2}$ & 1,3-butadiyne & 11 & $696.31 \pm 0.22$ & 696.03 & $696.09 \pm 0.55$ & 0.0024 & -0.43 & 0.58 & -0.09 & 0.06 \\
\hline $\mathrm{C}_{4} \mathrm{H}_{4}$ & bicyclo[1.1.0]but-1(3)-ene & 12 & $786.50 \pm 0.26$ & 785.85 & $785.75 \pm 0.31$ & 0.0054 & -0.41 & 0.30 & 0.01 & -0.10 \\
\hline $\mathrm{C}_{4} \mathrm{H}_{4}$ & butatriene & 13 & $845.05 \pm 0.21$ & 844.84 & $844.82 \pm 0.47$ & 0.0039 & -0.42 & 0.44 & -0.04 & -0.03 \\
\hline $\mathrm{C}_{4} \mathrm{H}_{4}$ & cyclobutadiene & 14 & $819.86 \pm 0.24$ & 819.37 & $819.30 \pm 0.42$ & 0.0024 & -0.41 & 0.37 & -0.02 & -0.06 \\
\hline $\mathrm{C}_{4} \mathrm{H}_{4}$ & ethynylethene & 15 & $853.17 \pm 0.22$ & 853.09 & $853.04 \pm 0.47$ & 0.0018 & -0.43 & 0.46 & -0.08 & -0.05 \\
\hline $\mathrm{C}_{4} \mathrm{H}_{4}$ & methylenecyclopropene & 16 & $829.23 \pm 0.24$ & 829.34 & $829.27 \pm 0.42$ & 0.0034 & -0.42 & 0.37 & -0.02 & -0.06 \\
\hline $\mathrm{C}_{4} \mathrm{H}_{4}$ & tetrahedrane & 17 & $792.47 \pm 0.29$ & 792.55 & $792.41 \pm 0.34$ & 0.0007 & -0.40 & 0.23 & 0.03 & -0.14 \\
\hline $\mathrm{C}_{4} \mathrm{H}_{6}$ & 1,2-butadiene & 18 & $999.53 \pm 0.23$ & 999.69 & $999.53 \pm 0.41$ & 0.0018 & -0.43 & 0.34 & -0.06 & -0.16 \\
\hline $\mathrm{C}_{4} \mathrm{H}_{6}$ & 1,3-butadiene & 19 & $1012.32 \pm 0.23$ & 1012.34 & $1012.18 \pm 0.41$ & 0.0015 & -0.43 & 0.34 & -0.06 & -0.16 \\
\hline $\mathrm{C}_{4} \mathrm{H}_{6}$ & 2-butyne & 20 & $1003.52 \pm 0.23$ & 1003.64 & $1003.46 \pm 0.30$ & 0.0012 & -0.44 & 0.36 & -0.10 & -0.18 \\
\hline $\mathrm{C}_{4} \mathrm{H}_{6}$ & bicyclo[1.1.0]butane & 21 & $985.37 \pm 0.27$ & 985.46 & $985.22 \pm 0.34$ & 0.0004 & -0.42 & 0.20 & -0.01 & -0.23 \\
\hline $\mathrm{C}_{4} \mathrm{H}_{6}$ & cyclobutene & 22 & $1000.70 \pm 0.25$ & 1000.86 & $1000.66 \pm 0.32$ & 0.0014 & -0.42 & 0.27 & -0.04 & -0.20 \\
\hline $\mathrm{C}_{4} \mathrm{H}_{6}$ & methylenecyclopropane & 23 & $992.40 \pm 0.25$ & 992.55 & $992.35 \pm 0.32$ & 0.0014 & -0.42 & 0.27 & -0.04 & -0.20 \\
\hline $\mathrm{C}_{4} \mathrm{H}_{8}$ & cyclobutane & 24 & $1149.37 \pm 0.26$ & 1149.61 & $1149.28 \pm 0.34$ & 0.0003 & -0.43 & 0.16 & -0.06 & -0.33 \\
\hline $\mathrm{C}_{4} \mathrm{H}_{8}$ & isobutene & 25 & $1158.67 \pm 0.24$ & 1158.92 & $1158.63 \pm 0.32$ & 0.0011 & -0.44 & 0.23 & -0.08 & -0.29 \\
\hline $\mathrm{C}_{5} \mathrm{H}_{4}$ & 1,4-pentadiyne & 26 & $986.65 \pm 0.28$ & 986.60 & $986.57 \pm 0.34$ & 0.0020 & -0.54 & 0.62 & -0.11 & -0.02 \\
\hline $\mathrm{C}_{5} \mathrm{H}_{4}$ & pentatetraene & 27 & $988.16 \pm 0.27$ & 987.75 & $987.78 \pm 0.61$ & 0.0050 & -0.52 & 0.58 & -0.04 & 0.02 \\
\hline $\mathrm{C}_{5} \mathrm{H}_{4}$ & spiropentadiene & 28 & $940.27 \pm 0.32$ & 940.33 & $940.27 \pm 0.38$ & 0.0015 & -0.51 & 0.44 & 0.01 & -0.05 \\
\hline
\end{tabular}


Table S21, contd.

\begin{tabular}{|c|c|c|c|c|c|c|c|c|c|c|}
\hline & \multirow[t]{2}{*}{ Molecule } & \multirow[t]{2}{*}{$\mathrm{MID}^{a}$} & \multicolumn{3}{|c|}{$E_{A, e^{b}}$} & \multirow[t]{2}{*}{$\Delta T_{1}\left(2_{s d}\right)^{c}$} & \multicolumn{4}{|c|}{$\mathrm{ATOMIC}(\mathrm{hc})-\mathrm{ATOMIC}^{d}$} \\
\hline & & & Best. est. & ATOMIC & ATOMIC(hc) & & $\operatorname{CCSD}(\mathrm{T})$ & h.o. & DBOC & sum \\
\hline $\mathrm{C}_{6} \mathrm{H}_{2}$ & 1,3,5-hexatriyne & 29 & $988.85 \pm 0.33$ & 988.21 & $988.34 \pm 0.83$ & 0.0031 & -0.64 & 0.89 & -0.11 & 0.13 \\
\hline $\mathrm{C}_{6} \mathrm{H}_{4}$ & butalene & 30 & $1099.88 \pm 0.38$ & 1099.80 & $1099.79 \pm 0.64$ & 0.0040 & -0.61 & 0.59 & 0.02 & 0.00 \\
\hline $\mathrm{C}_{6} \mathrm{H}_{4}$ & hexapentaene & 31 & $1131.92 \pm 0.32$ & 1131.05 & $1131.12 \pm 0.75$ & 0.0063 & -0.63 & 0.73 & -0.03 & 0.07 \\
\hline $\mathrm{C}_{6} \mathrm{H}_{4}$ & triafulvalene & 32 & $1082.98 \pm 0.38$ & 1083.07 & $1083.07 \pm 0.64$ & 0.0020 & -0.61 & 0.59 & 0.02 & 0.00 \\
\hline $\mathrm{C}_{6} \mathrm{H}_{6}$ & benzene & 33 & $1367.84 \pm 0.37$ & 1368.16 & $1368.05 \pm 0.63$ & 0.0010 & -0.63 & 0.56 & -0.03 & -0.10 \\
\hline
\end{tabular}

a Column "MID" reports the molecule index used in Figure 5.

${ }^{b}$ Best estimate (as defined in Sec. 5 and detailed in Table 6), ATOMIC/A*, and ATOMIC(hc)/A* values of the "bottom-of-the-well" total atomization energy (kcal/mol) using RI-MP2/cc-pVTZ geometries.

${ }^{c}$ Excess $T_{1}$ diagnostic as defined in Sec. 4.7 .

${ }^{d}$ Difference between ATOMIC $(\mathrm{hc})$ and ATOMIC $(\mathrm{kcal} / \mathrm{mol})$ and contributing components $\left(\mathrm{CCSD}(\mathrm{T}), \operatorname{specifically:~} C_{A, e}^{\mathrm{CCSD}(\mathrm{T})\{m\}}[M]+\right.$ $C_{A, e}^{\text {o.s.a. }\{m\}}[M]$ for model $\mathrm{A}^{*}$; higher-order electron correlation corrections, $C_{A, e}^{\text {h.o. }\{m\}}[M] ;$ DBOC, $\left.C_{A, e}^{\text {DBOC. }\{m\}}[M]\right) . A T O M I C($ hc $)$ and 